\title{
Stereocontrolled Synthesis of the Daphenylline Pentacyclic ACDEF Ring System
}

\author{
Sergi Jansana, Faïza Diaba* and Josep Bonjoch* \\ Laboratori de Química Orgànica, Facultat de Farmàcia, IBUB, \\ Universitat de Barcelona, Av. Joan XXIII s/n, 08028-Barcelona, Spain \\ E-mail: josep.bonjoch@ub.edu; faiza.diaba@ub.edu
}

\begin{abstract}
Contents
Experimental and NMR Data of compounds 1-18

S2-S16

Other experimental procedures

S17-S22

Copies of ${ }^{1} \mathrm{H}$ and ${ }^{13} \mathrm{C}$ NMR spectra of compounds 2-18

S23-S43
\end{abstract}

\section{General Experimental Procedure}

NMR spectra were recorded in $\mathrm{CDCl}_{3}$ and the chemical shifts of ${ }^{1} \mathrm{H}$ and ${ }^{13} \mathrm{C}$ NMR spectra are reported in ppm downfield $(\delta)$ from $\mathrm{Me}_{4} \mathrm{Si}$. All NMR data assignments are supported by gCOSY and gHSQC experiments. Analytical thin-layer chromatography was performed on $\mathrm{SiO}_{2}$ (Merck silica gel $60 \mathrm{~F}_{254}$ ), and the spots were located by UV light or with $1 \%$ aqueous $\mathrm{KMnO}_{4}$ solution. Chromatography refers to flash chromatography and was carried out on $\mathrm{SiO}_{2}$ (Carlo Erba silica gel 60A, 35-70 $\mu \mathrm{m}$ particle size). Drying of organic extracts during workup of reactions was performed over anhydrous $\mathrm{Na}_{2} \mathrm{SO}_{4}$. Solvent evaporation was accomplished with a rotatory evaporator. All yields refer to chromatographically and spectroscopically (NMR) pure material.

In the NMR assignment data throughout the supporting information, IUPAC systematic numbering is used for compounds 2-7, 11, and 12. Biosynthetic numbering was used for tetra- and pentacyclic compounds 8-10 and 13-18. 
<smiles>CC(=O)N(Cc1ccccc1)C1=C(C)c2ccccc2CC1</smiles>

Method A (from 1-methyl-2-tetralone): A mixture of 1-methyl-2-tetralone (1, $5.20 \mathrm{~g}, 32.45 \mathrm{mmol}$ ), benzylamine (4.26 mL, $38.9 \mathrm{mmol}, 1.2$ equiv) and molecular sieves ( $4 \AA, 5 \mathrm{~g})$ in dichloromethane $(10 \mathrm{~mL})$ was stirred at room temperature overnight. The reaction mixture was filtered through a short celite ${ }^{\circledR}$ pad and concentrated to afford the corresponding enamine as a yellowish oil (90\% conversion by ${ }^{1} \mathrm{H}$ NMR): ${ }^{1} \mathrm{H}$ NMR $(400 \mathrm{MHz}$, $\left.\mathrm{CDCl}_{3}\right) \delta$ 7.40-6.85 (m, 9H), $4.33(\mathrm{~s}, 2 \mathrm{H}), 2.71(\mathrm{t}, J=8 \mathrm{~Hz}), 2.35(\mathrm{t}, J=8 \mathrm{~Hz}), 1.91(\mathrm{t}, J$ $\left.=1.4 \mathrm{~Hz}, 3 \mathrm{H}, \mathrm{CH}_{3}\right)$. A solution of the above enamine in toluene $(83 \mathrm{~mL})$ was added to a solution of trichloroacetyl chloride $(4.35 \mathrm{~mL}, 39.0 \mathrm{mmol}, 1.2$ equiv) in toluene ( $83 \mathrm{~mL}$ ) dropwise at $0{ }^{\circ} \mathrm{C}$. After stirring for $1 \mathrm{~h}$ at room temperature the mixture was cooled again to $0{ }^{\circ} \mathrm{C}$ and a solution of triethylamine (13.7 mL, $97.4 \mathrm{mmol}, 3.0$ equiv) in toluene (135 $\mathrm{mL}$ ) was added dropwise and the mixture stirred at room temperature for an additional hour. A saturated solution of $\mathrm{Na}_{2} \mathrm{CO}_{3}(80 \mathrm{~mL})$ was added and after an additional hour the biphasic mixture was extracted with ether $(2 \times 200 \mathrm{~mL})$, and then with dichloromethane $(2 \times 50 \mathrm{~mL})$. The organic extract was washed with brine, dried, concentrated, and purified by chromatography (0-2.5\% EtOAc in hexane) to give trichloroacetamide 2 as a yellow oil $\left(9.01 \mathrm{~g}, 71 \%\right.$ over 2 steps): ${ }^{1} \mathrm{H}$ NMR $(400 \mathrm{MHz}$, $\left.\mathrm{CDCl}_{3}\right) \delta 7.36-7.29(\mathrm{~m}, 9 \mathrm{H}), 4.98$ and 4.61 (2 br d, $J=12.4 \mathrm{~Hz}, 1 \mathrm{H}$ each), 2.92 (br s, 1H), 2.71 (br s, 1H), 2.55 (br s, 1H), 2.32 (br s, 1H), 1.72 (br s, 3H); ${ }^{13} \mathrm{C}$ NMR (100 $\left.\mathrm{MHz}, \mathrm{CDCl}_{3}\right) \delta 160.9,135.9,135.3,134.5,134.4,132.9,129.5,128.4,128.1,127.9$, $127.2,126.4,124.2,56.0,29.3,28.4,15.5$. HRMS (ESI) $\mathrm{m} / \mathrm{z}:[\mathrm{M}+\mathrm{H}]^{+}$calcd for $\mathrm{C}_{20} \mathrm{H}_{19} \mathrm{Cl}_{3} \mathrm{NO} 394.0527$, found 394.0504

Method B (from $\beta$-tetralone): A mixture of $\beta$-tetralone $(5 \mathrm{~g}, 34.2 \mathrm{mmol}$ ), benzylamine (4.50 mL, $41.0 \mathrm{mmol}, 1.2$ equiv) and molecular sieves (4 $\AA, 5 \mathrm{~g})$ in dichloromethane (15 $\mathrm{mL}$ ) was stirred at room temperature overnight. The reaction mixture was filtered through a short celite ${ }^{\circledR}$ pad and concentrated to afford the corresponding enamine as a yellowish oil (100\% conversion by ${ }^{1} \mathrm{H}$ NMR): ${ }^{1} \mathrm{H}$ NMR $\left(400 \mathrm{MHz}, \mathrm{CDCl}_{3}\right) \delta$ 7.38-7.33 (m, $5 \mathrm{H}), 7.08-7.00(\mathrm{~m}, 2 \mathrm{H}), 6.87(\mathrm{~m}, 2 \mathrm{H}), 5.32(\mathrm{~s}, 1 \mathrm{H}), 4.26(\mathrm{~s}, 2 \mathrm{H}), 2.84(\mathrm{t}, J=7.6 \mathrm{~Hz}, 2 \mathrm{H})$, $2.33(\mathrm{t}, J=7.6 \mathrm{~Hz}, 2 \mathrm{H})$. To a solution of diisopropylamine $(8.6 \mathrm{~mL}, 61.5 \mathrm{mmol}, 1.8$ equiv) in THF $(165 \mathrm{~mL})$ at $-78{ }^{\circ} \mathrm{C}$ was added dropwise a $2.5 \mathrm{M}$ solution of BuLi in hexane $(21.9 \mathrm{~mL}, 54.7 \mathrm{mmol}, 1.6$ equiv). The mixture was stirred at this temperature for $15 \mathrm{~min}$ and transferred via a cannula to the reaction flask containing the previously prepared enamine in THF $(200 \mathrm{~mL})$ at $-78{ }^{\circ} \mathrm{C}$ and stirred for an additional $30 \mathrm{~min}$. Methyl iodide ( $4.5 \mathrm{~mL}, 71.8 \mathrm{mmol}, 2.1$ equiv) was added dropwise and the reaction was stirred for $2.5 \mathrm{~h}$ at $-78^{\circ} \mathrm{C}$. The reaction was allowed to reach room temperature, brine 
was added and the mixture was extracted with dichloromethane. The organics were dried, concentrated and used in the next step. Operating as above (method $A$ ), the acylation was performed using trichloroacetylchloride $(4.6 \mathrm{~mL}, 41.0 \mathrm{mmol}, 1.2$ equiv) and triethylamine (14.4 mL, $102.6 \mathrm{mmol}, 3.0$ equiv). After chromatography, trichloroacetamide 2 was obtained ( $8.4 \mathrm{~g}, 63 \%$ over 3 steps).

\section{3-Benzyl-9b-methyl-1,3,5,9b-tetrahydro-2H-benzo[e]indol-2-one (3)}

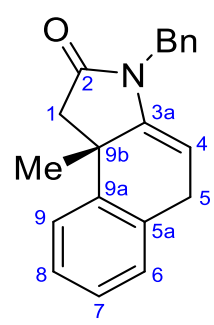

To a refluxing solution of $2(9.8 \mathrm{~g}, 24.8 \mathrm{mmol})$ in benzene $(350 \mathrm{~mL})$ was added a solution of $\mathrm{Bu}_{3} \mathrm{SnH}(21.4 \mathrm{~mL}, 79.5 \mathrm{mmol}, 3.2$ equiv) and AIBN (1.2 g, $7.45 \mathrm{mmol}, 0.3$ equiv) in benzene $(26 \mathrm{~mL})$ over $4 \mathrm{~h}$ using a syringe pump. The reaction mixture was concentrated and purified by chromatography (hexane to hexane/EtOAc $3: 1$ ) to give enamide 3 as a pale yellow oil $(4.98 \mathrm{~g}, 70 \%):{ }^{1} \mathrm{H}$ NMR $\left(400 \mathrm{MHz}, \mathrm{CDCl}_{3}\right) \delta$ 7.32-7.13 (m, $9 \mathrm{H}, \mathrm{ArH}), 5.09(\mathrm{dd}, J=6.4,2.2 \mathrm{~Hz}, 1 \mathrm{H}, \mathrm{H}-4), 4.86$ and $4.55(2 \mathrm{~d}, J=15.4 \mathrm{~Hz}, 1 \mathrm{H}$ each, $\mathrm{CH}_{2} \mathrm{Ph}$ ), 3.39 (br dd, $J=20.0,2.2 \mathrm{~Hz}, 1 \mathrm{H}, \mathrm{H}-5$ ), 3.28 (br dd, $J=20.0,6.4 \mathrm{~Hz}, 1 \mathrm{H}, \mathrm{H}-5$ ), 2.97 and $2.80(2 \mathrm{~d}, J=16.2 \mathrm{~Hz}, 1 \mathrm{H}$ each, $\mathrm{H}-1), 1.29$ (s, 3H, $\left.\mathrm{CH}_{3}\right) ;{ }^{13} \mathrm{C} \mathrm{NMR}(100 \mathrm{MHz}$,

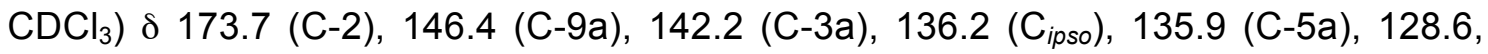
127.9, 127.4, 127.2, 126.7, 126.3, and 124.8 (C Ar), 96.2 (C-4), 43.8 (C-1), 43.7 $\left(\mathrm{CH}_{2} \mathrm{Ph}\right), 41.0$ (C-9b), $29.2(\mathrm{C}-5), 29.0\left(\mathrm{CH}_{3}\right)$. HRMS (ESI) m/z: $[\mathrm{M}+\mathrm{H}]^{+}$calcd for $\mathrm{C}_{20} \mathrm{H}_{20} \mathrm{NO} 290.1539$, found 290.1538 .

(1RS,9bRS)-1-Allyl-3-benzyl-9b-methyl-1,3,5,9b-tetrahydro-2H-benzo[e]indol-2one (4)

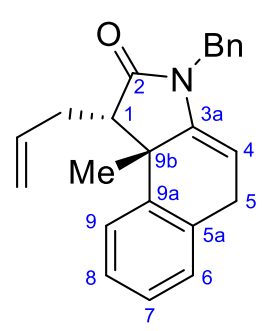

To a solution of $3(4.9 \mathrm{~g}, 17.0 \mathrm{mmol})$ in THF $(54 \mathrm{~mL})$ was added a solution of LHMDS (1 M in THF, $22 \mathrm{~mL}, 22.0 \mathrm{mmol}, 1.3$ equiv) dropwise at $-78^{\circ} \mathrm{C}$. After $30 \mathrm{~min}$, allylbromide $(2.9 \mathrm{~mL}, 33.9 \mathrm{mmol}, 2.0$ equiv) was added dropwise and the reaction was allowed to reach room temperature over $2.5 \mathrm{~h}$. The reaction was quenched with a saturated solution of $\mathrm{NH}_{4} \mathrm{Cl}(100 \mathrm{~mL})$ and extracted with ether $(3 \mathrm{x}$ $100 \mathrm{~mL}$ ). The organic extracts were dried, filtered through a silica gel pad and concentrated. The residue was purified by chromatography (hexane to hexane/EtOAc 9:1) affording 4 as a colorless solid (5.2 g, 94\%): mp 88-92 ${ }^{\circ} \mathrm{C} ;{ }^{1} \mathrm{H}$ NMR $(400 \mathrm{MHz}$, $\left.\mathrm{CDCl}_{3}\right) \delta 7.31-7.12(\mathrm{~m}, 9 \mathrm{H}, \mathrm{ArH}), 5.67$ (ddt, $\left.J=17.0,10.0,7.0 \mathrm{~Hz}, 1 \mathrm{H},=\mathrm{CH}\right), 5.08$ (dd, $J=5.6,2.4 \mathrm{~Hz}, 1 \mathrm{H}, \mathrm{H}-4), 4.89-4.84\left(\mathrm{~m}, 2 \mathrm{H},=\mathrm{CH}_{2}\right), 4.74$ and $4.65(2 \mathrm{~d}, J=15.3 \mathrm{~Hz}, 1 \mathrm{H}$ 
each, $\mathrm{CH}_{2} \mathrm{Ph}$ ), 3.44 (dd, $J=20.7,2.4 \mathrm{~Hz}, 1 \mathrm{H}, \mathrm{H}-5 \mathrm{eq}$ ), 3.35 (dd, $J=20.7,5.6 \mathrm{~Hz}, 1 \mathrm{H}, \mathrm{H}-$ 5ax), 2.79 (dd, $J=9.1,4.0 \mathrm{~Hz}, 1 \mathrm{H}, \mathrm{H}-1), 2.51$ and $2.10\left(2 \mathrm{~m}, 1 \mathrm{H}\right.$ each, $\left.\mathrm{CH}_{2}\right), 1.34$ (s, $\left.3 \mathrm{H}, \mathrm{CH}_{3}\right) ;{ }^{13} \mathrm{C}$ NMR (100 MHz, $\mathrm{CDCl}_{3}$ ) $\delta 175.9$ (C-2), 144.0 (C-9a), 137.9 (C-3a), 136.5 $\left(\mathrm{C}_{\text {ipso }}\right), 135.1(\mathrm{C}-5 \mathrm{a}), 134.2(=\mathrm{CH}), 128.4,128.3,127.4,127.3,126.8,126.1$, and 126.0 (C Ar), $116.9\left(=\mathrm{CH}_{2}\right), 96.5(\mathrm{C}-4), 52.8(\mathrm{C}-1), 43.9(\mathrm{C}-9 \mathrm{~b}), 43.4\left(\mathrm{CH}_{2} \mathrm{Ph}\right), 34.1\left(\mathrm{CH}_{2}\right)$, $31.1\left(\mathrm{CH}_{3}\right), 28.9(\mathrm{C}-5)$. HRMS (ESI) $\mathrm{m} / \mathrm{z}$ : $[\mathrm{M}+\mathrm{H}]^{+}$calcd for $\mathrm{C}_{23} \mathrm{H}_{24} \mathrm{NO} 330.1852$, found 330.1849 .

(1RS,3aSR,9bRS)-1-Allyl-3-benzyl-9b-methyl-1,3,3a,4,5,9b-hexahydro-2H benzo[e]indol-2-one (5)
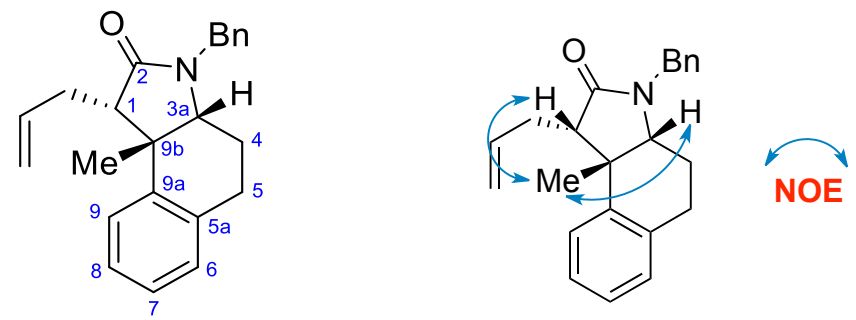

To a solution of enamide $4(4.8 \mathrm{~g}, 14.5 \mathrm{mmol})$ in glacial acetic acid $(145 \mathrm{~mL})$ was added sodium cyanoborohydride (4.6 g, $72.7 \mathrm{mmol}, 5.0$ equiv) portionwise. The mixture was stirred at room temperature for $48 \mathrm{~h}$, quenched carefully with a $\mathrm{Na}_{2} \mathrm{CO}_{3}$ saturated solution until the bubbling stopped and extracted with EtOAc $(3 \times 100 \mathrm{~mL})$. The resulting organic layer was washed with brine, dried, concentrated and purified by chromatography (hexane to hexane/EtOAc 3:1) to give lactam 5 as a white solid (3.62 g, 75\%): mp 94-97 ${ }^{\circ} \mathrm{C} ;{ }^{1} \mathrm{H}$ NMR (400 MHz, $\mathrm{CDCl}_{3}$ ) $\delta$ 7.36-7.06 (m, 9H, ArH), 5.92 (ddt, $J=17.0,10.1,7.0 \mathrm{~Hz}, 1 \mathrm{H},=\mathrm{CH}), 5.03$ and $4.12\left(2 \mathrm{~d}, J=14.8 \mathrm{~Hz}, 1 \mathrm{H}\right.$ each, $\left.\mathrm{CH}_{2} \mathrm{Ph}\right)$, 4.94 (ddt, $\left.J=10.0,1.8,1.2 \mathrm{~Hz}, 1 \mathrm{H},=\mathrm{CH}_{2}\right), 4.86\left(\mathrm{dq}, J=17.0,1.8 \mathrm{~Hz}, 1 \mathrm{H},=\mathrm{CH}_{2}\right), 3.17$ (dd, $J=11.7,4.2 \mathrm{~Hz}, 1 \mathrm{H}, \mathrm{H}-3 \mathrm{a}), 2.73(\mathrm{dt}, J=16.3,4.2 \mathrm{~Hz}, 1 \mathrm{H}, \mathrm{H}-5 \mathrm{eq}), 2.63(\mathrm{~m}, 1 \mathrm{H}, \mathrm{H}-$ $5 a x), 2.54(\mathrm{dd}, J=9.8,4.9 \mathrm{~Hz}, 1 \mathrm{H}, \mathrm{H}-1), 2.19-2.10\left(\mathrm{~m}, 2 \mathrm{H}, \mathrm{CH}_{2}\right), 2.08-2.02(\mathrm{~m}, 1 \mathrm{H}, \mathrm{H}-$ 4eq), 1.55 (qd, $J=11.7,4.2 \mathrm{~Hz}, 1 \mathrm{H}, \mathrm{H}-4 \mathrm{ax}), 1.32$ (s, 3H, CH $\left.\mathrm{CH}_{3}\right) \cdot{ }^{13} \mathrm{C}$ NMR $(100 \mathrm{MHz}$, $\left.\mathrm{CDCl}_{3}\right) \delta 175.1(\mathrm{C}-2), 138.8(\mathrm{C}-9 \mathrm{a}), 136.9(\mathrm{C}-5 \mathrm{a}), 136.8(=\mathrm{CH}), 136.4\left(\mathrm{C}_{\text {ipso }}\right), 128.8$, 128.7, 128.6, 128.3, 127.6, 126.4, 126.1 (C Ar), 115.7 (= $\left.\mathrm{CH}_{2}\right), 64.2$ (C-3a), 54.6 (C-1), $44.5\left(\mathrm{CH}_{2} \mathrm{Ph}\right), 42.3(\mathrm{C}-9 \mathrm{~b}), 35.7\left(\mathrm{CH}_{2} \mathrm{C}=\right), 32.4\left(\mathrm{CH}_{3}\right), 27.6(\mathrm{C}-5), 26.6(\mathrm{C}-4)$. HRMS (ESI) $\mathrm{m} / \mathrm{z}:[\mathrm{M}+\mathrm{H}]^{+}$calcd for $\mathrm{C}_{23} \mathrm{H}_{26} \mathrm{NO} 332.2009$, found 332.2007 . 
<smiles>C[C@]12CCCc3ccccc3[C@]1(C)CCC2=O</smiles>

A solution of 9-BBN (0.5 M in THF, $10.3 \mathrm{~mL}, 5.13 \mathrm{mmol}, 2.0$ equiv) and alkene 5 (850 $\mathrm{mg}, 2.6 \mathrm{mmol})$ was stirred at room temperature for $3 \mathrm{~h}$. The mixture was cooled to $0^{\circ} \mathrm{C}$ and a cold $\left(0{ }^{\circ} \mathrm{C}\right)$ solution of aqueous $\mathrm{NaOH}(2 \mathrm{M}, 77 \mathrm{~mL}, 153.9 \mathrm{mmol}, 60$ equiv) and $\mathrm{H}_{2} \mathrm{O}_{2}$ (30 wt $\%, 31.7 \mathrm{~mL}, 307.7 \mathrm{mmol}, 120$ equiv) was added dropwise. After $10 \mathrm{~min}$, the reaction mixture was vigorously stirred overnight at room temperature. Water $(15 \mathrm{~mL})$ was added and the mixture was extracted with EtOAc $(2 \times 15 \mathrm{~mL})$. The organic extracts were dried, concentrated, and purified by chromatography (hexane to hexane/EtOAc 1:1) to give alcohol 6 as a colorless oil (805 mg, 90\%): ${ }^{1} \mathrm{H}$ NMR $\left(400 \mathrm{MHz}, \mathrm{CDCl}_{3}\right) \delta$ 7.36-7.26 (m, $5 \mathrm{H}, \mathrm{ArH}), 7.20(\mathrm{~m}, 1 \mathrm{H}, \mathrm{ArH}), 7.11-7.05(\mathrm{~m}, 3 \mathrm{H}, \mathrm{ArH}) 5.02$ and $4.11(\mathrm{~d}, J=14.8 \mathrm{~Hz}, 1 \mathrm{H}$ each, $\mathrm{CH}_{2} \mathrm{Ph}$ ), $3.61\left(\mathrm{t}, J=5.6 \mathrm{~Hz}, 2 \mathrm{H}, \mathrm{CH}_{2} \mathrm{OH}\right.$ ), $3.48(\mathrm{br} \mathrm{s}, 1 \mathrm{H}, \mathrm{OH}), 3.14$ (dd, $J=12.1$, $4.5 \mathrm{~Hz}, 1 \mathrm{H}, \mathrm{H}-3 \mathrm{a}$ ), 2.73 (dt, $J=16.4,4.0 \mathrm{~Hz}, 1 \mathrm{H}, \mathrm{H}-5 \mathrm{eq}), 2.62$ (m, 1H, H-5ax), 2.56 (dd, $J=10.4,3.6 \mathrm{~Hz}, 1 \mathrm{H}, \mathrm{H}-1), 2.17(\mathrm{dq}, J=12.1,4.5 \mathrm{~Hz}, 1 \mathrm{H}, \mathrm{H}-4 \mathrm{eq}), 1.82-1.64(\mathrm{~m}, 2 \mathrm{H}, \mathrm{H}-$ 2'), 1.48 (qd, $J=12.1,4.5 \mathrm{~Hz}, 1 \mathrm{H}, \mathrm{H}-4 \mathrm{ax}), 1.40$ (m, $\left.1 \mathrm{H}, \mathrm{H}-1^{\prime}\right), 1.29$ (s, 3H, $\mathrm{CH}_{3}$ ), 1.33$1.23\left(\mathrm{~m}, 1 \mathrm{H}, \mathrm{H}-1^{\prime}\right) ;{ }^{13} \mathrm{C}$ NMR (100 MHz, CDCl$\left.{ }_{3}\right) \delta 176.4$ (C-2), 138.9 (C-9a), 136.5 (C5a), 136.2 ( $\left.\mathrm{C}_{\text {ipso }}\right), 128.6,128.5,128.2,128.1,127.5,126.4$, and 125.9 (C Ar), 64.3 (C3a), $62.2\left(\mathrm{CH}_{2} \mathrm{OH}\right), 53.4(\mathrm{C}-1), 44.4\left(\mathrm{CH}_{2} \mathrm{Ph}\right), 42.7(\mathrm{C}-9 \mathrm{~b}), 32.0\left(\mathrm{CH}_{3}\right), 31.2(\mathrm{C}-2), 28.6$ (C-1'), 27.7 (C-5), 26.7 (C-4). HRMS (ESI) m/z: [M+H] $]^{+}$calcd for $\mathrm{C}_{23} \mathrm{H}_{28} \mathrm{NO}_{2} 350.2115$, found 350.2111 .

\section{(1RS,3aSR,9bRS)-3-Benzyl-9b-methyl-2-oxo-2,3,3a,4,5,9b-hexahydro-1H-}

\section{benzo[e]indol-1-yl)propanoic acid (7)}

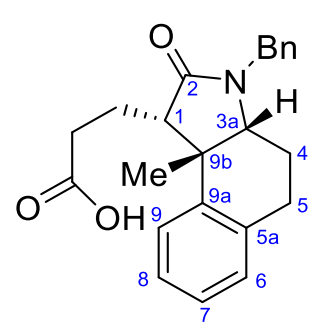

To a solution of alcohol $6(1.47 \mathrm{~g}, 4.24 \mathrm{mmol})$ in acetonitrile (43 $\mathrm{mL}$ ) were added a $\mathrm{pH} 7$ phosphate buffered solution $(29 \mathrm{~mL})$, TEMPO (100 mg, $0.63 \mathrm{mmol}, 0.15$ equiv), $80 \% \mathrm{NaClO}_{2}$ (959 mg, $8.48 \mathrm{mmol}, 80 \%$ in weight, 2.0 equiv), and $10 \%$ aqueous $\mathrm{NaClO}$ (4.10 mL, $5.51 \mathrm{mmol}, 1.3$ equiv). After stirring at room temperature for $5 \mathrm{~h}$, the reaction mixture was diluted with saturated aqueous $\mathrm{Na}_{2} \mathrm{SO}_{3}(100 \mathrm{~mL})$ and stirred for an additional $10 \mathrm{~min}$. The resulting mixture was acidified with $1 \mathrm{M}$ aqueous $\mathrm{HCl}(350 \mathrm{~mL})$ and extracted with EtOAc $(3 \times 350 \mathrm{~mL})$. The organic extracts were washed with brine $(2 \times 50 \mathrm{~mL})$, dried, concentrated and purified by chromatography (hexane to hexane/EtOAc $1: 1$ ), affording acid 7 as a white solid 


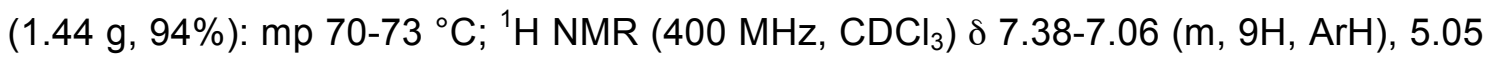
and $4.11\left(2 \mathrm{~d}, J=14.8 \mathrm{~Hz}, 1 \mathrm{H}\right.$ each, $\mathrm{CH}_{2} \mathrm{Ph}$ ), $3.18(\mathrm{dd}, J=12.4,4.4 \mathrm{~Hz}, 1 \mathrm{H}, \mathrm{H}-3 \mathrm{a}), 2.77$ (dt, $J=16.3,3.8 \mathrm{~Hz}, 1 \mathrm{H}, \mathrm{H}-5 \mathrm{eq}), 2.69-2.53$ (m, 4H, H-1, H-5ax and H-2'), 2.22 (dq, $J=$ 12.4, 4.0, 1H, H-4eq), 1.77 (m, 1H, H-1') 1.51 (qd, J = 12.6, 4.0 Hz, 1H, H-4ax), 1.37 (m, $\left.1 \mathrm{H}, \mathrm{H}-1^{\prime}\right), 1.30\left(\mathrm{~s}, 3 \mathrm{H}, \mathrm{CH}_{3}\right) ;{ }^{13} \mathrm{C}$ NMR (100 MHz, $\left.\mathrm{CDCl}_{3}\right) \delta 176.0(\mathrm{COOH}), 175.9$ (C-2), 138.1 (C-9a), 136.1 (C-5a), 136.0 (C 126.7, and 126.3 (C Ar), 64.8 (C-3a), 52.3 (C-1), $44.7\left(\mathrm{CH}_{2} \mathrm{Ph}\right), 42.3$ (C-9b), 32.5 (C2'), $32.2\left(\mathrm{CH}_{3}\right), 27.8$ (C-5), 26.9 (C-4 and C-1'). HRMS (ESI) m/z: $[\mathrm{M}+\mathrm{H}]^{+}$calcd for $\mathrm{C}_{23} \mathrm{H}_{26} \mathrm{NO}_{3} 364.1907$, found 364.1913.

\section{Keto lactam 8}

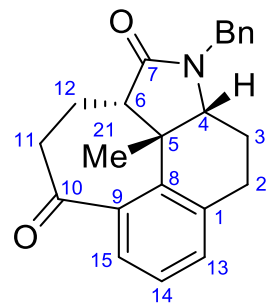

Acid 7 (960 mg, $2.64 \mathrm{mmol})$ was treated with $\mathrm{SOCl}_{2}(3.75 \mathrm{~mL}, 51.5$ mmol, 19 equiv) and the flask was air-dried after gas evolution. This process was repeated four times. The residue was dissolved in dichloromethane $(90 \mathrm{~mL})$ and $\mathrm{AlCl}_{3}(3.87 \mathrm{~g}, 29 \mathrm{mmol}, 11$ equiv) was added in one portion. The resulting mixture was stirred at $45^{\circ} \mathrm{C}$ for 1 $\mathrm{h}$ and concentrated. After chromatography (hexane/EtOAc 1:0 to $0: 1$ ) ketone 8 was obtained as a colorless oil $(865 \mathrm{mg}, 95 \%) ;{ }^{1} \mathrm{H} \mathrm{NMR}\left(400 \mathrm{MHz}, \mathrm{CDCl}_{3}\right) \delta$ 7.38-7.19 (m, 8H, ArH), 5.21 and $3.89\left(2 \mathrm{~d}, J=14.8 \mathrm{~Hz}, 1 \mathrm{H}\right.$ each, $\left.\mathrm{CH}_{2} \mathrm{Ph}\right), 3.46$ (br t, $J$ $=3.0 \mathrm{~Hz}, 1 \mathrm{H}, \mathrm{H}-4), 2.76(\mathrm{~m}, 2 \mathrm{H}, \mathrm{H}-11), 2.71(\mathrm{~m}, 1 \mathrm{H}, \mathrm{H}-2 \mathrm{ax}), 2.67(\mathrm{~m}, 1 \mathrm{H}, \mathrm{H}-6), 2.64$ (ddd, $J=16.0,5.0,2.8 \mathrm{~Hz}, \mathrm{H}-2 \mathrm{eq}), 2.47$ (dq, $J=14.8,4.0 \mathrm{~Hz}, 1 \mathrm{H}, \mathrm{H}-12), 2.17$ (dq, $J=$ 14.4, 3.0, 1H, H-3eq), 1.81 (dddd, $J=14.5,13.2,5.0,2.5 \mathrm{~Hz}, 1 \mathrm{H}, \mathrm{H}-3 \mathrm{ax}), 1.61(\mathrm{~m}, 1 \mathrm{H}$, $\mathrm{H}-12), 1.40$ (s, 3H, H-21); ${ }^{13} \mathrm{C}$ NMR (100 MHz, CDCl 3 ) $\delta 209.3$ (C-10), 176.1 (C-7), 141.0 (C-8), 136.0 (Cipso), 135.7 (C-9), 135.6 (C-1), 132.7, 128.8, 128.1, 127.7, 127.6, and 127.0 (C Ar), 61.4 (C-4), 52.7 (C-6), $44.2\left(\mathrm{CH}_{2} \mathrm{Ph}\right), 43.0(\mathrm{C}-5), 38.9$ (C-11), 31.9 (C-21), 24.4 (C-2), 22.8 (C-12), 20.3 (C-3). HRMS (ESI) m/z: [M+H] calcd for $\mathrm{C}_{23} \mathrm{H}_{24} \mathrm{NO}_{2} 346.1802$, found 346.1800 .

\section{Tetracyclic alcohol 9}

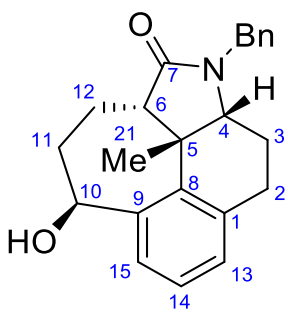

To a solution of ketone $8(1 \mathrm{~g}, 2.89 \mathrm{mmol})$ in $\mathrm{MeOH}(25 \mathrm{~mL})$ was added $\mathrm{NaBH}_{4}\left(548 \mathrm{mg}, 14.47 \mathrm{mmol}, 5.0\right.$ equiv) at $0{ }^{\circ} \mathrm{C}$ and the mixture was stirred at room temperature overnight. The reaction was quenched with $1 \mathrm{~N} \mathrm{HCl}$, concentrated and extracted with EtOAc $(3 \mathrm{x}$ $25 \mathrm{~mL})$. The organic extracts were washed with brine $(2 \times 25 \mathrm{~mL})$, 
dried and concentrated to afford alcohol 9 (943 mg, 94\%) as a colorless solid, enough pure to be used in the next step without purification. An analytical sample was purified (hexane to hexane/EtOAc 1:3): $\mathrm{mp} 117-119{ }^{\circ} \mathrm{C} ;{ }^{1} \mathrm{H}$ NMR $\left(400 \mathrm{MHz}, \mathrm{CDCl}_{3}\right) \delta$ 7.36-7.28 $(\mathrm{m}, 5 \mathrm{H}, \mathrm{ArH}), 7.10-7.04(\mathrm{~m}, 3 \mathrm{H}, \mathrm{ArH}), 5.09$ and $4.08(2 \mathrm{~d}, J=14.8 \mathrm{~Hz}, 1 \mathrm{H}$ each, $\mathrm{CH}_{2} \mathrm{Ph}$ ), 5.02 (dd, $J=6.0,2.4 \mathrm{~Hz}, 1 \mathrm{H}, \mathrm{H}-10$ ), 3.25 (dd, $J=11.2,4.4 \mathrm{~Hz}, 1 \mathrm{H}, \mathrm{H}-4$ ), 3.13 (dd, $J=11.8,1.8 \mathrm{~Hz}, 1 \mathrm{H}, \mathrm{H}-6$ ), 2.72 (ddd, $J=15.6,5.3,3.6 \mathrm{~Hz}, 1 \mathrm{H}, \mathrm{H}-2 \mathrm{eq}$ ), 2.56 (ddd, $J=15.6,11.2,3.6 \mathrm{~Hz}, 1 \mathrm{H}, \mathrm{H}-2 \mathrm{ax}), 2.24-2.10(\mathrm{~m}, 3 \mathrm{H}, \mathrm{H}-3 \mathrm{eq}, \mathrm{H}-11$ and $\mathrm{H}-12), 2.04(\mathrm{~m}$, $1 \mathrm{H}, \mathrm{H}-11), 1.48(\mathrm{~s}, 3 \mathrm{H}, \mathrm{H}-21), 1.33(\mathrm{~m}, 1 \mathrm{H}, \mathrm{H}-12), 1.22$ (qd, $J=11.2,4.0 \mathrm{~Hz}, 1 \mathrm{H}, \mathrm{H}-$ 3ax); ${ }^{13} \mathrm{C}$ NMR (100 MHz, $\mathrm{CDCl}_{3}$ ) $\delta 176.3$ (C-7), 140.1 (C-8), 139.5 (C-9 and C-1), 136.6 (Cipso), 129.8, 129.6, 128.7, 127.9, 127.5, and 126.2 (Ar), 76.5 (C-10), 68.1 (C4), 51.2 (C-6), 44.4 (C-5), $44.1\left(\mathrm{CH}_{2} \mathrm{Ph}\right), 34.7$ (C-21), 33.5 (C-11), 28.9 (C-2), 27.2 (C3), $26.5(\mathrm{C}-12)$. HRMS (ESI) $\mathrm{m} / \mathrm{z}$ : $[\mathrm{M}+\mathrm{H}]^{+}$calcd for $\mathrm{C}_{23} \mathrm{H}_{26} \mathrm{NO}_{2} 348.1958$, found 348.1955 .

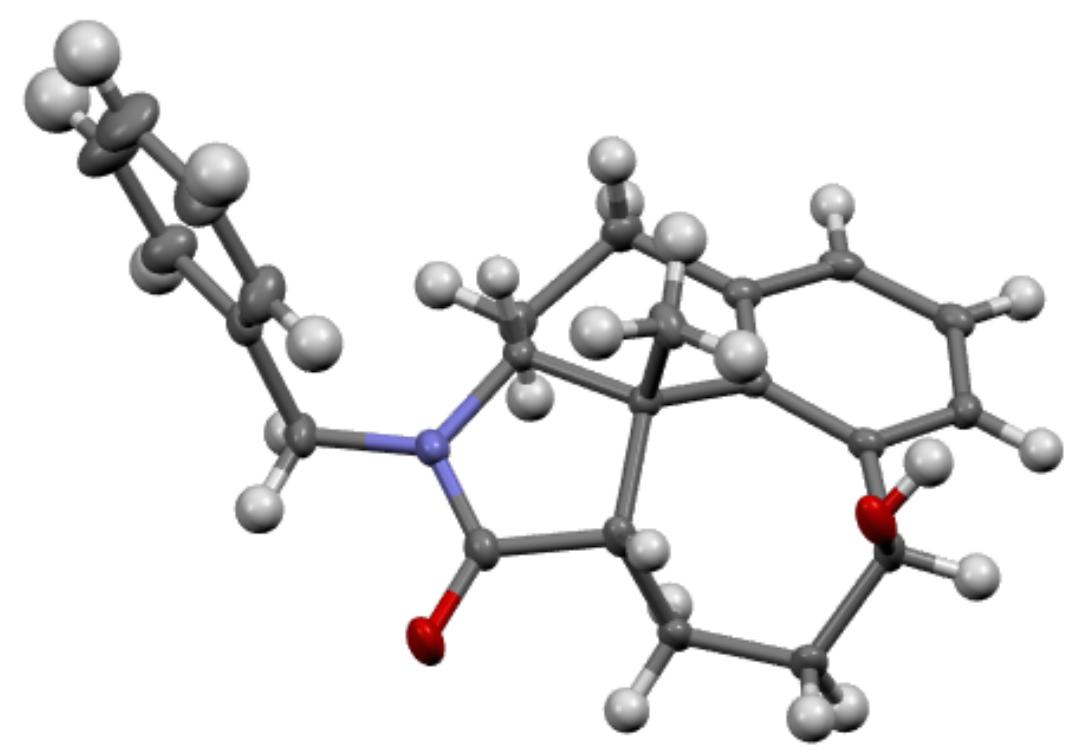

\section{X-Ray Data for Tetracyclic Alcohol 9}

\section{Xanthate 9a}

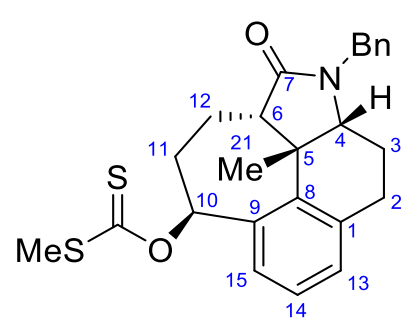

$\mathrm{CS}_{2}(66 \mu \mathrm{L}, 1.1 \mathrm{mmol}, 17$ equiv) and $\mathrm{NaH}(50-72 \%$ dispersion in mineral oil, $22 \mathrm{mg}, 0.5 \mathrm{mmol}, 8.0$ equiv) were successively added to a solution of $9(23 \mathrm{mg}, 0.07 \mathrm{mmol}, 1.0$ equiv) in THF (1 mL) at room temperature. After the reaction mixture was stirred for $1.5 \mathrm{~h}$, Mel $(87 \mu \mathrm{L}, 1.39 \mathrm{mmol})$ was 
added. The resulting mixture was stirred for $1 \mathrm{~h}$ at room temperature. The reaction was quenched with saturated aqueous $\mathrm{NH}_{4} \mathrm{Cl}(0.5 \mathrm{~mL})$ at $0{ }^{\circ} \mathrm{C}$ and extracted with EtOAc (3 $x 5 \mathrm{~mL})$. The organics were washed with brine $(5 \mathrm{~mL})$, dried, concentrated and purified by chromatography (hexane to hexane/EtOAc 1:1) to afford xanthate $9 \mathrm{a}$ as a colorless oil (27 mg, 94\%): ${ }^{1} \mathrm{H}$ NMR (400 MHz, $\left.\mathrm{CDCl}_{3}\right) \delta$ 7.38-7.29 (m, 6H, ArH), $7.13(\mathrm{~m}, 2 \mathrm{H}$, ArH), $6.83(\mathrm{dd}, J=6.2,1.8 \mathrm{~Hz}, 1 \mathrm{H}, \mathrm{H}-10), 5.09$ and $4.12(2 \mathrm{~d}, J=15.2 \mathrm{~Hz}, 1 \mathrm{H}$ each, $\mathrm{CH}_{2} \mathrm{Ph}$ ), 3.26 (dd, $J=12.0,4.4 \mathrm{~Hz}, 1 \mathrm{H}, \mathrm{H}-4$ ), 3.07 (dd, $\left.J=12.0,1.6 \mathrm{~Hz}, 1 \mathrm{H}, \mathrm{H}-6\right), 2.74$ (dt, $J=16.0,3.2 \mathrm{~Hz}, 1 \mathrm{H}, \mathrm{H}-2), 2.57\left(\mathrm{~m}, 4 \mathrm{H}, \mathrm{H}-2\right.$ and $\mathrm{CH}_{3}$ ), 2.37 (dddd, $J=15.6,11.6$, 6.2, $4.2 \mathrm{~Hz}, 1 \mathrm{H}, \mathrm{H}-11), 2.24(\mathrm{~m}, 2 \mathrm{H}, \mathrm{H}-12$ and $\mathrm{H}-3 \mathrm{eq}), 2.14$ (dtd, $J=15.6,6.2,1.8 \mathrm{~Hz}$, $1 \mathrm{H}, \mathrm{H}-11), 1.45$ (m, 1H, H-12), 1.39 (s, 3H, H-21), 1.17 (qd, J = 12.0, 4.4 Hz, 1H, H3ax); ${ }^{13} \mathrm{C}$ NMR (100 MHz, $\mathrm{CDCl}_{3}$ ) $\delta 214.9$ (C=S), 175.6 (C-7), 141.1 (C-8), 139.8 (C-1), $136.4\left(\mathrm{C}_{i p s o}\right), 134.3$ (C-9), 131.8, 130.4, 128.7, 127.9, 127.6, and 126.1 (C Ar), 86.3 (C10), 68.8 (C-4), 50.9 (C-6), 44.5 (C-5), $44.1\left(\mathrm{CH}_{2} \mathrm{Ph}\right), 34.6$ (C-21), 31.6 (C-11), 29.2 (C2), 27.9 (C-3), $26.7(\mathrm{C}-12), 19.4\left(\mathrm{CH}_{3}\right)$. HRMS (ESI) m/z: $[\mathrm{M}+\mathrm{H}]^{+}$calcd for $\mathrm{C}_{25} \mathrm{H}_{28} \mathrm{NO}_{2} \mathrm{~S}_{2}$ [438.1556, found 438.1554 .

\section{Tetracyclic bromide 9b}

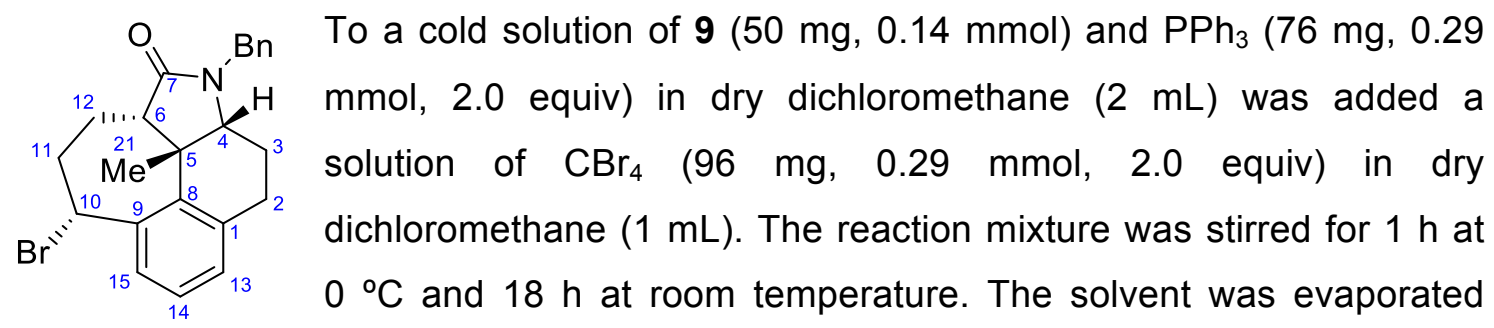
and the residue was purified by chromatography (hexane to hexane/EtOAc 1:1) to afford bromide $9 \mathrm{~b}$ as a colorless oil $(32 \mathrm{mg}, 54 \%$ ). The elimination product 10 (see below) was also obtained in $22 \%$ yield. Compound $9 \mathrm{~b}:{ }^{1} \mathrm{H} \mathrm{NMR}\left(400 \mathrm{MHz}, \mathrm{CDCl}_{3}\right) \delta$ 7.76 and $7.06(2 \mathrm{~d}, J=7.6 \mathrm{~Hz}, 1 \mathrm{H}$ each, ArH), 7.38-7.28 (m, 5H, ArH), $7.19(\mathrm{t}, J=7.6$ $\mathrm{Hz}, 1 \mathrm{H}, \mathrm{ArH}), 5.46(\mathrm{dd}, J=12.2,5.8 \mathrm{~Hz}, 1 \mathrm{H}, \mathrm{H}-10), 5.12$ and $4.06(2 \mathrm{~d}, J=14.8 \mathrm{~Hz}, 1 \mathrm{H}$ each, $\mathrm{CH}_{2} \mathrm{Ph}$ ), $3.28(\mathrm{dd}, J=12.4,4.8 \mathrm{~Hz}, 1 \mathrm{H}, \mathrm{H}-4), 2.73(\mathrm{dt}, J=15.6,3.6 \mathrm{~Hz}, 1 \mathrm{H}, \mathrm{H}-$ 2eq), 2.55-2.47 (m, 3H, H-11, H-6 and H-2ax), 2.28 (dq, $J=12.4,4.8 \mathrm{~Hz}, 1 \mathrm{H}, \mathrm{H}-3 \mathrm{eq}$ ), 2.17 (br dd, $J=13.6,7.6,1 \mathrm{H}, \mathrm{H}-12$ ), $2.03(\mathrm{td}, J=12.2,7.6 \mathrm{~Hz}, 1 \mathrm{H}, \mathrm{H}-11), 1.35(\mathrm{~s}, 3 \mathrm{H}$, $\mathrm{H}-21), 1.22(\mathrm{~m}, 1 \mathrm{H}, \mathrm{H}-12), 1.05$ (qd, $J=12.4,4.8 \mathrm{~Hz}, 1 \mathrm{H}, \mathrm{H}-3 \mathrm{ax}) ;{ }^{13} \mathrm{C}$ NMR $(100 \mathrm{MHz}$, $\left.\mathrm{CDCl}_{3}\right) \delta 174.5$ (C-7), 138.0 (C-8), 136.6 (C-9), 136.5 (C-1), $136.2\left(\mathrm{C}_{\text {ipso }}\right), 128.9,128.8$, 127.9, 127.7, 127.6, and 127.0 (C Ar), 68.1 (C-4), 52.8 (C-6), 51.6 (C-10), 44.4 $\left(\mathrm{CH}_{2} \mathrm{Ph}\right.$ ), 42.7 (C-5), 39.2 (C-11), 31.3 (C-21), 28.7 (C-3 and C-2), 28.1 (C-12). HRMS (ESI) $m / z:[M+H]^{+}$calcd for $\mathrm{C}_{23} \mathrm{H}_{25} \mathrm{BrNO} 410.1114$, found 410.1109 . 


\section{Tetracyclic alkene 10}

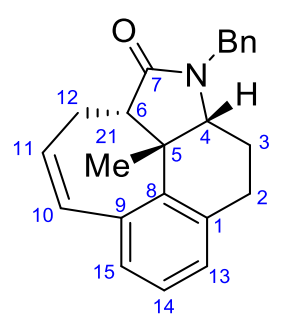

To a solution of alcohol 9 (842 $\mathrm{mg}, 2.42 \mathrm{mmol}, 1.0$ equiv) in 1,2dichloroethane $(11 \mathrm{~mL})$ was added $\operatorname{lnCl}_{3}(161 \mathrm{mg}, 0.73 \mathrm{mmol}, 0.3$ equiv) and the mixture was stirred at $80^{\circ} \mathrm{C}$ for $4 \mathrm{~h}$. The reaction was quenched with $\mathrm{NaHCO}_{3}$ aqueous solution $(25 \mathrm{~mL})$ and extracted with diethyl ether $(3 \times 25 \mathrm{~mL})$. The organic extracts were dried, concentrated, and purified by chromatography (hexane to EtOAc) to give alkene 12 as a colorless oil (575 mg, 70\%, over 2 steps): ${ }^{1} \mathrm{H}$ NMR (400 MHz, $\left.\mathrm{CDCl}_{3}\right) \delta$ 7.36-7.27 (m, 5H, ArH), $7.10(\mathrm{t}, J=7.6 \mathrm{~Hz}, 1 \mathrm{H}, \mathrm{ArH}), 6.95(\mathrm{br} \mathrm{d}, J=7.6 \mathrm{~Hz}$, $1 \mathrm{H}, \mathrm{ArH}), 6.92$ (d, $J=7.6 \mathrm{~Hz}, 1 \mathrm{H}, \mathrm{ArH}), 6.67$ (br d, $J=10.2 \mathrm{~Hz}, 1 \mathrm{H}, \mathrm{H}-10), 6.19$ (ddd, $J$ $=10.2,8.0,5.7 \mathrm{~Hz}, 1 \mathrm{H}, \mathrm{H}-11), 5.18$ and $3.95\left(2 \mathrm{~d}, J=14.8 \mathrm{~Hz}, 1 \mathrm{H}\right.$ each, $\left.\mathrm{CH}_{2} \mathrm{Ph}\right), 3.34$ (t, $J=3.2 \mathrm{~Hz}, 1 \mathrm{H}, \mathrm{H}-4), 2.95(\mathrm{dd}, J=13.4,5.7 \mathrm{~Hz}, 1 \mathrm{H}, \mathrm{H}-6), 2.73(\mathrm{~m}, 1 \mathrm{H}, \mathrm{H}-2), 2.60-$ $2.53(\mathrm{~m}, 2 \mathrm{H}, \mathrm{H}-2$ and $\mathrm{H}-12$ ), 2.12 (dq, $J=14.4,3.2 \mathrm{~Hz}, 1 \mathrm{H}, \mathrm{H}-3 \mathrm{eq}), 1.98$ (tdd, $J=13.4$, 5.7, $2.4 \mathrm{~Hz}, 1 \mathrm{H}, \mathrm{H}-12$ ), 1.81 (tdd, J = 14.4, 3.2, 3.2 Hz, 1H, H-3ax), 1.39 (s, 3H, H-21); ${ }^{13} \mathrm{C}$ NMR (100 MHz, CDCl 3 ) $\delta 176.7$ (C-7), 138.7 (C-8), 138.6 (C-9), 136.3 (C 1), 133.9 (C-10), 128.7, 128.5, 128.2, 128.1, 127.8, 127.5, and 125.6 (C Ar), 62.3 (C4), 61.1 (C-6), 44.1 ( $\mathrm{CH}_{2} \mathrm{Ph}$ ), 43.4 (C-5), 30.3 (C-21), 26.1 (C-12), 25.2 (C-2), 21.0 (C3). HRMS (ESI) $m / z$ : $[M+H]^{+}$calcd for $\mathrm{C}_{23} \mathrm{H}_{24} \mathrm{NO} 330.1852$, found 330.1853 .

\section{Allylic Alcohol 11}

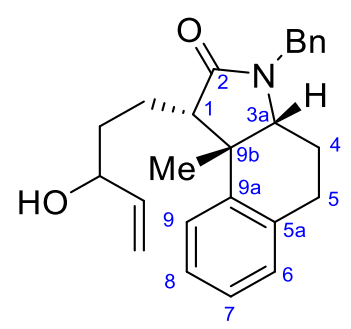

To a solution of alcohol 6 ( $88 \mathrm{mg}, 0.25 \mathrm{mmol}, 1.0$ equiv) in dichloromethane $(1.5 \mathrm{~mL})$ at $0{ }^{\circ} \mathrm{C}$ were added successively under vigorous stirring TEMPO ( $1 \mathrm{mg}, 0.005 \mathrm{mmol}, 0.02$ equiv) and $\mathrm{NaBr}$ (26 mg, $0.25 \mathrm{mmol}, 1.0$ equiv). To the resulting mixture was added a solution of $\mathrm{NaHCO}_{3}(49 \mathrm{mg}, 0.58 \mathrm{mmol}, 2.3$ equiv) and $10 \% \mathrm{NaClO}(0.23 \mathrm{~mL}, 0.38 \mathrm{mmol}, 1.5$ equiv) in water $(1.5$ $\mathrm{mL})$. After stirring at room temperature for $3 \mathrm{~h}$, the mixture was extracted with ether $(4 \mathrm{x}$ $5 \mathrm{~mL}$ ). The combined organics were washed with a $10 \% \mathrm{NaHSO}_{4}$ aqueous solution and $\mathrm{KI}(4 \%)$, and then with brine. Drying and concentration gave the corresponding aldehyde as a colorless oil (87 mg), which was used in the next step without further purification.

Vinylmagnesium bromide ( $1 \mathrm{M}$ in THF, $0.43 \mathrm{~mL}, 0.43 \mathrm{mmol}, 3.0$ equiv) was added to a solution of aldehyde (50 mg, $0.14 \mathrm{mmol}, 1.0$ equiv) in ether $(1 \mathrm{~mL})$ at $-78{ }^{\circ} \mathrm{C}$ and the reaction mixture was stirred for $30 \mathrm{~min}$. The suspension was allowed to reach room 
temperature and stirred for $2.5 \mathrm{~h}$, quenched with $2 \mathrm{~N} \mathrm{HCl}(0.2 \mathrm{~mL})$, and extracted with ether $(2 \times 5 \mathrm{~mL})$. The combined organics were dried and concentrated to give an equimolecular mixture of epimeric alcohols 11, as a brownish oil (54 mg, $98 \%$ over 2 steps): ${ }^{1} \mathrm{H}$ NMR $\left(400 \mathrm{MHz}, \mathrm{CDCl}_{3}\right) \delta$ 7.37-7.29 (m, 13H, ArH), 7.12-7.08 (m, 5H, ArH), 5.95-5.77 (m, $\left.2 \mathrm{H},=\mathrm{CH}_{2}\right)$, 5.33-5.14 (m, 2H, $\left.=\mathrm{CH}_{2}\right), 5.09-5.00\left(\mathrm{~m}, 4 \mathrm{H},=\mathrm{CH}\right.$ and $\left.\mathrm{CH}_{2} \mathrm{Ph}\right)$, 4.19-4.10 (m, $4 \mathrm{H}, \mathrm{CH}_{2} \mathrm{Ph}$ and $\left.\mathrm{CHOH}\right), 3.15(\mathrm{~m}, 2 \mathrm{H}, \mathrm{H}-3 \mathrm{a}), 2.73(\mathrm{~m}, 2 \mathrm{H}, \mathrm{H}-5), 2.68-2.58$ $(\mathrm{m}, 4 \mathrm{H}, \mathrm{H}-5$ and $\mathrm{H}-1), 2.17(\mathrm{~m}, 2 \mathrm{H}, \mathrm{H}-4), 1.78-1.66\left(\mathrm{~m}, 4 \mathrm{H}, \mathrm{CH}_{2} \mathrm{CHOH}\right), 1.47(\mathrm{~m}, 2 \mathrm{H}$, $\mathrm{H}-4), 1.30$ (s, 3H, $\left.\mathrm{CH}_{3}\right), 1.29$ (s, 3H, $\mathrm{CH}_{3}$ epi), 1.27-1.22 (m, 4H, $\left.\mathrm{CH}_{2}-\mathrm{CH}-1\right) ;{ }^{13} \mathrm{C} \mathrm{NMR}$ $\left(100 \mathrm{MHz}, \mathrm{CDCl}_{3}\right) \delta 176.5$ and $176.4(\mathrm{C}-2), 141.4(=\mathrm{CH}), 140.9\left(\mathrm{C}-7 \mathrm{a}^{1}\right), 136.6(\mathrm{C}-7 \mathrm{a})$, 136.3 (Cipso), 128.7, 128.6, 128.4, 128.3, 128.2, 127.7, 126.6, 126.5, 126.1, and 126.0 $(\mathrm{Ph}), 113.8\left(=\mathrm{CH}_{2}\right), 72.3$ and $72.2(\mathrm{CHOH}), 64.6$ and $64.5\left(\mathrm{C}-2 \mathrm{a}^{1}\right), 53.3(\mathrm{C}-2 \mathrm{a}), 44.6 \times 2$ $\left(\mathrm{CH}_{2} \mathrm{Ph}\right), 42.8 \times 2$ (C-9b), 35.4 and $35.1\left(\mathrm{CH}_{2} \mathrm{CHOH}\right), 32.2 \times 2\left(\mathrm{CH}_{3}\right.$ and $\mathrm{CH}_{3}$ epi), 27.8x4 (C-5 and $\mathrm{CH}_{2}-\mathrm{C}-1$ ), 26.9 (C-4). HRMS (ESI) $\mathrm{m} / \mathrm{z}$ : $[\mathrm{M}+\mathrm{H}]^{+}$calcd for $\mathrm{C}_{25} \mathrm{H}_{29} \mathrm{NO}_{2}$, 376.2271 , found 376.2274 .

\section{(2aRS,2a $\left.{ }^{1} S R, 4 S R\right)-1-B e n z y l-2 a^{1}-$ methyl-4-propyl-2a ${ }^{1}, 3,4,8,9,9 a-h e x a h y d r o-$ 1H-naphtho[2,1,8-cde]indol-2(2aH)-one (12b)}

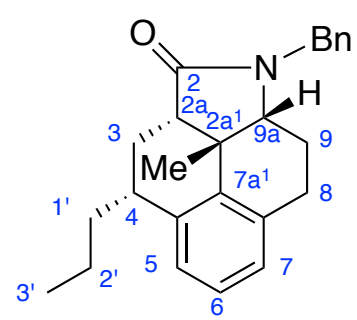

purified by chromatography (hexane/EtOAc $9: 1$ to $1: 3$ ), affording a mixture of alkenes 12a ( $\mathrm{dr} 3: 2)$ as a colorless oil $(25 \mathrm{mg}, 84 \%)$, used in the next transformation. To a solution of $12 \mathrm{a}$ (15 mg, $0.04 \mathrm{mmol}, 1.0$ equiv) in $\mathrm{MeOH}(1 \mathrm{~mL})$ was added $\mathrm{Pd} / \mathrm{C} 10 \%$ (7 mg). The reaction was stirred overnight at room temperature under a $\mathrm{H}_{2}$ atmosphere (1 atm). The mixture was filtered on a celite ${ }^{\circledR}$ pad and concentrated to afford a mixture of tetracyclic compounds $12 \mathrm{~b}(\mathrm{dr} \mathrm{3:2)}$ as a colorless oil (13 mg, 87\%). Major epimer (depicted in the Figure): ${ }^{1} \mathrm{H}$ NMR $\left(400 \mathrm{MHz} \mathrm{CDCl}_{3}\right) \delta$ 7.35-6.97 (m, 8H, ArH), 5.00 and $3.98\left(2 \mathrm{~d}, J=15.0 \mathrm{~Hz}, 1 \mathrm{H}\right.$ each, $\left.\mathrm{CH}_{2} \mathrm{Ph}\right), 3.51$ (dd, $\left.J=11.2,6.0 \mathrm{~Hz}, 1 \mathrm{H}, \mathrm{H}-9 \mathrm{a}\right), 2.90-$ $2.82(\mathrm{~m}, 1 \mathrm{H}, \mathrm{H}-4), 2.78(\mathrm{dd}, \mathrm{J}=10.6,8.2 \mathrm{~Hz}, 1 \mathrm{H}, \mathrm{H}-2 \mathrm{a}), 2.60-2.51(\mathrm{~m}, 3 \mathrm{H}, 2 \mathrm{H}-8$ and $\mathrm{H}-$ 3), 2.34-2.24 (m, 1H, H-9), 2.04 and 1.56-1.42 (2m, 1H each, $\left.\mathrm{H}-1{ }^{\prime}\right), 1.66(\mathrm{~m}, 2 \mathrm{H}, \mathrm{H}-$ 2'), $1.32(\mathrm{~m}, 1 \mathrm{H}, \mathrm{H}-9), 1.23\left(\mathrm{~s}, 3 \mathrm{H}, \mathrm{CH}_{3}\right), 1.10(\mathrm{~m}, 1 \mathrm{H}, \mathrm{H}-3) 1.03\left(\mathrm{t}, J=7.2 \mathrm{~Hz}, 3 \mathrm{H}, \mathrm{CH}_{3}\right)$; ${ }^{13} \mathrm{C}$ NMR (100 MHz, $\mathrm{CDCl}_{3}$ ) $\delta 176.1$ (C-2), 143.0 (C-7a'), 139.4 (C-4a) 137.4 (C-7a), 
$136.6\left(\mathrm{C}_{i p s o}\right), 128.6,127.9,127.4,125.8$, and 122.6 (C Ar), 63.8 (C-9a), 50.2 (C-2a), $44.2\left(\mathrm{CH}_{2} \mathrm{Ph}\right), 38.5(\mathrm{C}-4), 37.6\left(\mathrm{C}-2 \mathrm{a}^{1}\right), 35.2\left(\mathrm{CH}_{3}\right), 34.5\left(\mathrm{C}-1^{\prime}\right), 31.2(\mathrm{C}-3), 27.5(\mathrm{C}-9)$, 26.2 (C-8), 20.1 (C-2'), $14.5\left(\mathrm{CH}_{3}\right)$. Minor epimer: 7.35-6.97 (m, 8H, ArH), 4.94 and $4.03\left(2 \mathrm{~d}, J=15.0 \mathrm{~Hz}, 1 \mathrm{H}\right.$ each, $\left.\mathrm{CH}_{2} \mathrm{Ph}\right), 3.50(\mathrm{t}, J=3.2 \mathrm{~Hz}, 1 \mathrm{H}, \mathrm{H}-9 \mathrm{a}), 2.90-2.82(\mathrm{~m}$, $1 \mathrm{H}, \mathrm{H}-2 \mathrm{a}), 2.63(\mathrm{~m}, 1 \mathrm{H}, \mathrm{H}-4), 2.60-2.51(\mathrm{~m}, 2 \mathrm{H}, \mathrm{H}-8), 2.43$ (ddd, $J=13.2,6.8,3.6 \mathrm{~Hz}$, $1 \mathrm{H}, \mathrm{H}-3), 2.34-2.24(\mathrm{~m}, 1 \mathrm{H}, \mathrm{H}-9), 1.66$ and 1.56-1.42 (2 $\mathrm{m}, 1 \mathrm{H}$ each, $\left.\mathrm{H}-1^{\prime}\right), 1.56-1.42$ (m, 3H, H-3 and 2H-2'), $1.27\left(\mathrm{~s}, 3 \mathrm{H}, \mathrm{CH}_{3}\right), 0.96\left(\mathrm{t}, \mathrm{J}=7.2 \mathrm{~Hz}, 4 \mathrm{H}, \mathrm{CH}_{3}\right.$ and $\left.\mathrm{H}-9\right) .{ }^{13} \mathrm{C}$

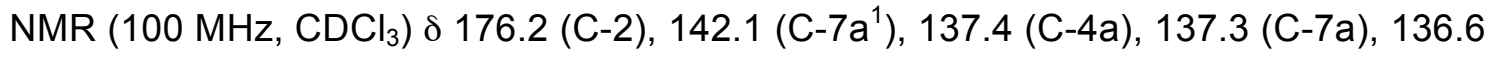
$\left(\mathrm{C}_{i p s o}\right), 128.6,127.9,127.4,125.7,125.4$, and 125.2 (C Ar), 65.4 (C-9a), 47.5 (C-2a), $44.4\left(\mathrm{CH}_{2} \mathrm{Ph}\right), 37.1\left(\mathrm{C}-2 \mathrm{a}^{1}\right), 36.1\left(\mathrm{C}-1^{\prime}\right), 35.2(\mathrm{C}-4), 32.5\left(\mathrm{CH}_{3}\right), 30.4(\mathrm{C}-3), 30.1(\mathrm{C}-9)$, $26.9(\mathrm{C}-8), 21.2\left(\mathrm{C}-2^{\prime}\right), 14.2\left(\mathrm{CH}_{3}\right)$. HRMS (ESI) $\mathrm{m} / \mathrm{z}$ : $[\mathrm{M}+\mathrm{H}]^{+}$calcd for $\mathrm{C}_{25} \mathrm{H}_{30} \mathrm{NO}$ 360.5109 , found 360.5104 .

\section{Ketone 13 (via epoxide 10a)}
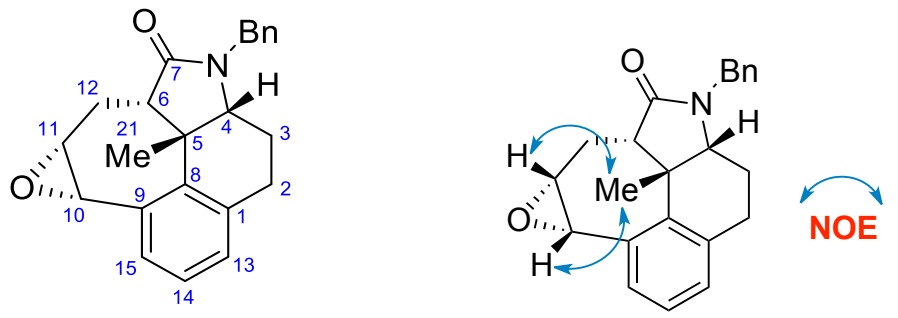

To a solution of alkene $10(510 \mathrm{mg}, 1.55 \mathrm{mmol})$ in dichloromethane $(27 \mathrm{~mL})$ was added $m$-CPBA (535 mg, $3.10 \mathrm{mmol}, 2.0$ equiv) and the mixture was stirred overnight at 40 ${ }^{\circ} \mathrm{C}$. An aqueous $0.4 \mathrm{M} \mathrm{Na}_{2} \mathrm{~S}_{2} \mathrm{O}_{3}$ in $1 \mathrm{~N} \mathrm{NaHCO}_{3}$ solution $(30 \mathrm{~mL})$ was added and stirred at room temperature for $30 \mathrm{~min}$. The mixture was extracted with EtOAc $(3 \times 20 \mathrm{~mL})$ and the organics were washed with brine $(2 \times 10 \mathrm{~mL})$, dried and concentrated to afford the corresponding epoxide $10 \mathrm{a}$ as a white oil, enough pure to be used in the next step without purification: ${ }^{1} \mathrm{H}$ NMR $\left(400 \mathrm{MHz}, \mathrm{CDCl}_{3}\right) \delta 7.41$ (br d, $\left.J=7.6 \mathrm{~Hz}, 1 \mathrm{H}, \mathrm{ArH}\right), 7.37-$ $7.28(\mathrm{~m}, 5 \mathrm{H}, \mathrm{ArH}), 7.18(\mathrm{t}, J=7.6 \mathrm{~Hz}, 1 \mathrm{H}, \mathrm{ArH}), 7.03(\mathrm{br} \mathrm{d}, J=7.6 \mathrm{~Hz}, 1 \mathrm{H}, \mathrm{ArH}), 5.15$ and $3.83\left(2 \mathrm{~d}, J=14.8 \mathrm{~Hz}, 1 \mathrm{H}\right.$ each, $\left.\mathrm{CH}_{2} \mathrm{Ph}\right), 4.06(\mathrm{~d}, J=4.4 \mathrm{~Hz}, 1 \mathrm{H}, \mathrm{H}-10), 3.47$ (br t, $J=3.0 \mathrm{~Hz}, 1 \mathrm{H}, \mathrm{H}-4$ ), 3.42 (ddd, $J=8.4,4.4,4.4 \mathrm{~Hz}, 1 \mathrm{H}, \mathrm{H}-11), 2.84-2.73(\mathrm{~m}, 2 \mathrm{H}, \mathrm{H}-12$ and $\mathrm{H}-6), 2.66-2.61(\mathrm{~m}, 2 \mathrm{H}, \mathrm{H}-2), 2.19$ (dq, $J=14.6,3.0 \mathrm{~Hz}, 1 \mathrm{H}, \mathrm{H}-3 \mathrm{eq}), 1.83$ (dddd, $J$ $=14.6,13.0,5.8,3.0 \mathrm{~Hz}, 1 \mathrm{H}, \mathrm{H}-3 \mathrm{ax}), 1.60(\mathrm{~s}, 3 \mathrm{H}, \mathrm{H}-21), 0.96(\mathrm{td}, J=13.0,8.4 \mathrm{~Hz}, 1 \mathrm{H}$, $\mathrm{H}-12) ;{ }^{13} \mathrm{C}$ NMR $\left(100 \mathrm{MHz}, \mathrm{CDCl}_{3}\right) \delta 175.9$ (C-7), $136.0\left(\mathrm{C}_{\text {ipso }}\right), 135.8$ (C-8), 135.5 (C9), 134.2 (C-1), 129.7, 129.3, 128.7, 128.1, 127.6, and 126.8 (C Ar), $61.6(\mathrm{C}-4), 54.1$ (C-10), 52.3 (C-11), 49.0 (C-6), $44.1\left(\mathrm{CH}_{2} \mathrm{Ph}\right), 42.7$ (C-5), 30.6 (C-21), 29.3 (C-12), 
24.5 (C-2), 20.2 (C-3). HRMS (ESI) m/z: $[\mathrm{M}+\mathrm{H}]^{+}$calcd for $\mathrm{C}_{23} \mathrm{H}_{24} \mathrm{NO}_{2} 346.1802$, found 346.1805 .

The above epoxide $10 \mathrm{a}(505 \mathrm{mg}, 1.46 \mathrm{mmol}, 1.0$ equiv) was
dissolved in benzene $(9 \mathrm{~mL})$ and $\mathrm{Znl}_{2}$ was added (233 mg, 0.73
mmol, 0.5 equiv). The mixture was stirred at $80^{\circ} \mathrm{C}$ for $1 \mathrm{~h}$, cooled to
room temperature and washed with water. The organic phase was EtOAc) to give ketone 13 as a pale yellow oil (432 mg, 81\% over two steps): ${ }^{1} \mathrm{H}$ NMR $\left(400 \mathrm{MHz}, \mathrm{CDCl}_{3}\right) \delta$ 7.39-7.29 (m, 5H, ArH), 7.12-7.00 (m, 3H, ArH), 5.15 and $4.06(2 \mathrm{~d}$, $J=14.8 \mathrm{~Hz}, 1 \mathrm{H}$ each, $\left.\mathrm{CH}_{2} \mathrm{Ph}\right), 4.13$ and $3.44(2 \mathrm{~d}, J=15.6 \mathrm{~Hz}, 1 \mathrm{H}$ each, $\mathrm{H}-10), 3.36$ (dd, $J=10.8,4.4 \mathrm{~Hz}, 1 \mathrm{H}, \mathrm{H}-4), 2.87(\mathrm{~m}, 2 \mathrm{H}, \mathrm{H}-12$ and $\mathrm{H}-6), 2.76$ (ddd, $J=16.4,6.0$, $3.4 \mathrm{~Hz}, 1 \mathrm{H}, \mathrm{H}-2 \mathrm{eq}$ ), 2.59 (ddd, $J=16.4,11.7,3.1 \mathrm{~Hz}, 1 \mathrm{H}, \mathrm{H}-2 \mathrm{ax}$ ), 2.51 (dd, $J=16.4$, $13.8 \mathrm{~Hz}, 1 \mathrm{H}, \mathrm{H}-12), 2.24(\mathrm{~m}, 1 \mathrm{H}, \mathrm{H}-3), 1.40$ (s, 3H, H-21), $1.28(\mathrm{~m}, 1 \mathrm{H}, \mathrm{H}-3) ;{ }^{13} \mathrm{C} \mathrm{NMR}$

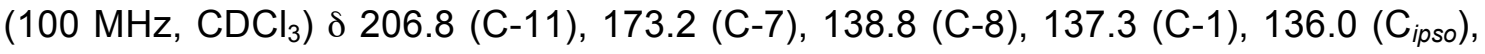
132.8 (C-9), 130.2, 128.8, 128.7, 127.9, 127.8, and 126.9 (C Ar), 66.7 (C-4), 51.5 (C6), 50.4 (C-10), $44.5\left(\mathrm{CH}_{2} \mathrm{Ph}\right), 42.3$ (C-5), 41.0 (C-12), 31.4 (C-21), 27.8 (C-2), 27.1 (C3). HRMS (ESI) m/z: $[\mathrm{M}+\mathrm{H}]^{+}$calcd for $\mathrm{C}_{23} \mathrm{H}_{24} \mathrm{NO}_{2} 346.1802$, found 346.1804.

When the transformation of ketone 8 to isomeric ketone 13 was carried out without purification of the intermediate, the overall yield for the four steps was $51 \%$.

\section{Tetracyclic $\gamma$-keto ester 14}
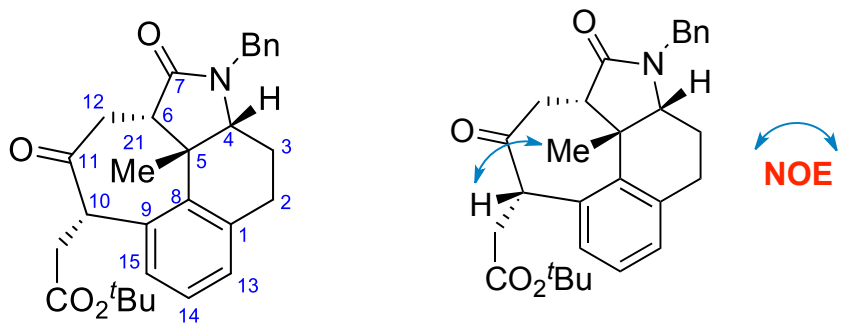

To a solution of ketone 13 (110 $\mathrm{mg}, 0.32 \mathrm{mmol}, 1.0$ equiv) in THF (2 mL) was added LHMDS ( $1 \mathrm{M}$ in THF, $0.4 \mathrm{~mL}, 0.38 \mathrm{mmol}, 1.2$ equiv) dropwise at $-78^{\circ} \mathrm{C}$. After $30 \mathrm{~min}$ of stirring at $-78{ }^{\circ} \mathrm{C}$, tert-butyl iodoacetate $(58 \mu \mathrm{L}, 0.38 \mathrm{mmol}, 1.2$ equiv) was added dropwise and the reaction was allowed to reach room temperature over $4 \mathrm{~h}$. The reaction was quenched with a saturated solution of $\mathrm{NH}_{4} \mathrm{Cl}(2 \mathrm{~mL})$ and extracted with EtOAc $(3 \times 10 \mathrm{~mL})$. The organic extracts were washed with brine $(2 \times 5 \mathrm{~mL})$, dried, concentrated and purified by chromatography (hexane to hexane/EtOAc 1:1), affording ester 14 as a pale colorless oil (64 mg, 83\%, brsm 91\%; dr 3:1): ${ }^{1} \mathrm{H}$ NMR (400 MHz, 
$\left.\mathrm{CDCl}_{3}\right) \delta 7.40-7.30(\mathrm{~m}, 5 \mathrm{H}, \mathrm{ArH}), 7.11(\mathrm{br} \mathrm{t}, J=7.6 \mathrm{~Hz}, 1 \mathrm{H}, \mathrm{ArH}), 7.05$ and 6.97 (two br $\mathrm{d}, J=7.6 \mathrm{~Hz}, 1 \mathrm{H}$ each, $\mathrm{ArH}), 5.15$ and $4.11\left(2 \mathrm{~d}, J=14.8 \mathrm{~Hz}, 1 \mathrm{H}\right.$ each, $\left.\mathrm{CH}_{2} \mathrm{Ph}\right), 4.71$ (dd, $J=8.4,6.0 \mathrm{~Hz}, 1 \mathrm{H}, \mathrm{H}-10$ ), 3.36 (dd, $J=12.4,4.6 \mathrm{~Hz}, 1 \mathrm{H}, \mathrm{H}-4), 3.16$ (dd, $J=5.6$, $3.6 \mathrm{~Hz}, 1 \mathrm{H}, \mathrm{H}-6), 3.12(\mathrm{~m}, 1 \mathrm{H}, \mathrm{H}-1$ '), 2.94 (dd, J = 18.2, $2.2 \mathrm{~Hz}, 1 \mathrm{H}, \mathrm{H}-12), 2.78$ (dd, $J=$ 16.6, $6.0 \mathrm{~Hz}, 1 \mathrm{H}, \mathrm{H}-1^{\prime}$ ), 2.76 (dt, $J=14.8,3.0 \mathrm{~Hz}, 1 \mathrm{H}, \mathrm{H}-2 \mathrm{eq}$ ), 2.56 (br td, $J=14.8,3.0$ $\mathrm{Hz}, 1 \mathrm{H}, \mathrm{H}-2 \mathrm{ax}$ ), 2.43 (dd, $J=18.2,13.8 \mathrm{~Hz}, 1 \mathrm{H}, \mathrm{H}-12$ ), 2.33 (dq, $J=12.4,3.0 \mathrm{~Hz}, 1 \mathrm{H}$, $\mathrm{H}-3 \mathrm{eq}$ ), 1.51 (s, 3H, H-21), 1.43 (s, 9H, ${ }^{t} \mathrm{Bu}$ ), 1.05 (qd, $J=12.4,4.6 \mathrm{~Hz}, 1 \mathrm{H}, \mathrm{H}-3 \mathrm{ax}$ ); ${ }^{13} \mathrm{C}$ NMR (100 MHz, CDCl ${ }_{3}$ ) $\delta 205.4$ (C-11), 172.8 (C-7), 171.0 (CO), 139.4 (C-8), 138.4 (C1), 136.1 ( $\left.\mathrm{C}_{\text {ipso }}\right), 134.3(\mathrm{C}-9), 128.8,128.7,127.9,127.8,126.8$, and 124.7 (C Ar), 81.0 (C), 68.1 (C-4), 51.6 (C-6), 49.2 (C-10), 44.5 ( $\left.\mathrm{CH}_{2} \mathrm{Ph}\right), 42.4$ (C-5), 40.9 (C-12), 32.7 (C21 and $\left.\mathrm{C}-1^{\prime}\right), 28.9$ (C-3 and C-2), $28.0\left(\mathrm{CH}_{3}\right)$. HRMS (ESI) m/z: $[\mathrm{M}+\mathrm{H}]^{+}$calcd for $\mathrm{C}_{29} \mathrm{H}_{34} \mathrm{NO}_{4} 460.2482$, found 460.2480 .

\section{Ester 15}

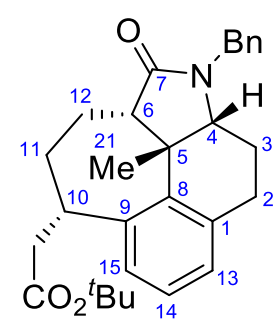

To a solution of keto ester $14(160 \mathrm{mg}, 0.35 \mathrm{mmol})$ in ethanol $(3 \mathrm{~mL})$ was added tosylhydrazine ( $85 \mathrm{mg}, 0.45 \mathrm{mmol}, 1.3$ equiv) and the mixture was stirred at room temperature in a sealed tube overnight. The solvent was evaporated, the residue was dissolved in a mixture of $\mathrm{THF} / \mathrm{H}_{2} \mathrm{O}(4: 1,8 \mathrm{~mL})$ and $\mathrm{NaBH}_{4}(53 \mathrm{mg}, 1.4 \mathrm{mmol}, 4.0$ equiv) was added. The reaction mixture was stirred at $66^{\circ} \mathrm{C}$ for $3 \mathrm{~h}$ and diluted in water and $n$-pentane. The aqueous layer was extracted three times with $n$ pentane $(3 \times 10 \mathrm{~mL})$ and the combined organic layers were washed successively with sat. $\mathrm{NaHCO}_{3}, 1 \mathrm{~N} \mathrm{HCl}$ and brine. The organic phase was dried, concentrated and purified by chromatography (hexane/EtOAc 95:5 to EtOAc), affording ester 15 as a yellowish oil (196 mg, 5:1 mixture of epimers $80 \%$ over two steps): ${ }^{1} \mathrm{H}$ NMR (400 MHz, $\left.\mathrm{CDCl}_{3}\right) \delta$ 7.36-7.29 (m, 5H, ArH), 7.11 and 7.00 (2br t, $J=7.6 \mathrm{~Hz}, 1 \mathrm{H}$ each, ArH), 5.13 and $4.07\left(2 \mathrm{~d}, J=14.8 \mathrm{~Hz}, 1 \mathrm{H}\right.$ each, $\left.\mathrm{CH}_{2} \mathrm{Ph}\right), 3.50(\mathrm{~m}, 1 \mathrm{H}, \mathrm{H}-10), 3.27(\mathrm{dd}, J=12.4,4.0$ $\mathrm{Hz}, 1 \mathrm{H}, \mathrm{H}-4), 2.80\left(\mathrm{dd}, J=15.6,8.4 \mathrm{~Hz}, 1 \mathrm{H}, \mathrm{H}-1^{\prime}\right), 2.70(\mathrm{dt}, J=15.6,4.0 \mathrm{~Hz}, 1 \mathrm{H}, \mathrm{H}-$ 2eq), $2.61\left(\mathrm{dd}, J=15.6,6.6 \mathrm{~Hz}, 1 \mathrm{H}, \mathrm{H}-1^{\prime}\right), 2.58-2.42(\mathrm{~m}, 3 \mathrm{H}, \mathrm{H}-12, \mathrm{H}-6$ and $\mathrm{H}-2), 2.26$ (br dq, $J=12.4,4.0 \mathrm{~Hz}, 1 \mathrm{H}, \mathrm{H}-3 \mathrm{eq}$ ), 2.17 (br dd, $J=13.4,7.0 \mathrm{~Hz}, 1 \mathrm{H}, \mathrm{H}-12$ ), 1.92 (m, $1 \mathrm{H}, \mathrm{H}-11$ ), 1.41 (s, 3H, H-21), 1.37 (s, 9H, ${ }^{t} \mathrm{Bu}$ ), 1.25 (m, 1H, $\mathrm{H}-11$ ), 1.07 (qd, $J=12.4$, $4.0 \mathrm{~Hz}, 1 \mathrm{H}, \mathrm{H}-3 \mathrm{ax}) ;{ }^{13} \mathrm{C}$ NMR (100 MHz, $\mathrm{CDCl}_{3}$ ) $\delta 175.3$ (C-7), 171.6 (CO), 140.0 (C-8), 138.9 (C-1), 138.3 (C-9), 136.5 ( $\left.\mathrm{C}_{\text {ipso }}\right), 128.7,127.9,127.5,127.4,126.4$, and 122.9 (C Ar), 80.5 (C), 68.3 (C-4), 53.2 (C-6), 44.3 ( $\left.\mathrm{CH}_{2} \mathrm{Ph}\right), 42.9$ (C-5), 39.8 (C-1'), 35.7 (C-10), 
33.4 (C-11), 31.5 (C-21), 29.0 (C-2), 28.7 (C-3), $28.0\left(\mathrm{CH}_{3}\right), 27.1$ (C-12). HRMS (ESI) $\mathrm{m} / \mathrm{z}:[\mathrm{M}+\mathrm{H}]^{+}$calcd for $\mathrm{C}_{29} \mathrm{H}_{36} \mathrm{NO}_{3} 446.2690$, found 446.2685 .

\section{Pentacyclic keto lactam 16}

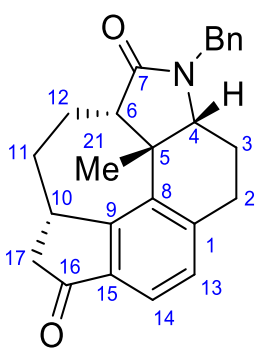

Ester $15(135 \mathrm{mg}, 0.030 \mathrm{mmol}, 5: 1$ mixture of epimers at $\mathrm{C}-10)$ was dissolved in a mixture of dichloromethane:trifluoroacetic acid (1:1, 9 $\mathrm{mL}$ ) and stirred for $45 \mathrm{~min}$ at room temperature. After removal of the solvent the residue was dissolved in dichloromethane $(4 \mathrm{~mL})$ and $\mathrm{SOCl}_{2}(0.11 \mathrm{~mL}, 1.51 \mathrm{mmol}, 5.0$ equiv $)$ was added. The reaction mixture was stirred at room temperature for $1 \mathrm{~h}$, concentrated, dissolved in dichloromethane $(8 \mathrm{~mL})$, and $\mathrm{AlCl}_{3}(202 \mathrm{mg}, 1.51 \mathrm{mmol}, 5.0$ equiv) was added in one portion. The resulting mixture was stirred at $45^{\circ} \mathrm{C}$ for $1 \mathrm{~h}$ and then concentrated. The residue was purified by chromatography (hexane/EtOAc 9:1 to EtOAc). The first eluate gave minor amounts of epi-16 (13 mg, 10\%) and pentacyclic ketone 16 was subsequently eluted as an oil (112 mg, 86\%).

16: ${ }^{1} \mathrm{H}$ NMR (400 MHz, $\left.\mathrm{CDCl}_{3}\right) \delta 7.55(\mathrm{~d}, J=7.6 \mathrm{~Hz}, 1 \mathrm{H}, \mathrm{H}-14), 7.38-7.29(\mathrm{~m}, 5 \mathrm{H}, \mathrm{ArH})$, $7.17(\mathrm{~d}, J=7.6 \mathrm{~Hz}, 1 \mathrm{H}, \mathrm{H}-13), 5.12$ and $4.11\left(2 \mathrm{~d}, J=14.8 \mathrm{~Hz}, 1 \mathrm{H}\right.$ each, $\left.\mathrm{CH}_{2} \mathrm{Ph}\right), 3.55$ (br q, J = 8.0 Hz, 1H, H-10), 3.31 (dd, $J=12.0,4.6 \mathrm{~Hz}, 1 \mathrm{H}, \mathrm{H}-4$ ), 2.95 (dd, $J=19.0,8.0$ $\mathrm{Hz}, 1 \mathrm{H}, \mathrm{H}-17), 2.80$ (dt, $J=16.0,3.6 \mathrm{~Hz}, 1 \mathrm{H}, \mathrm{H}-2 \mathrm{eq}), 2.59(\mathrm{~m}, 2 \mathrm{H}, \mathrm{H}-6$ and H-2ax), 2.38-2.29 (m, 3H, H-17, H-11 and H-3eq), 2.22 (br dd, $J=13.0,7.4 \mathrm{~Hz}, 1 \mathrm{H}, \mathrm{H}-12), 1.34$ (s, 3H, H-21), 1.38-1.21 (m, 2H, H-12 and H-11), $1.17(\mathrm{qd}, J=12.0,3.6 \mathrm{~Hz}, 1 \mathrm{H}, \mathrm{H}-$ 3ax); ${ }^{13} \mathrm{C}$ NMR (100 MHz, CDCl ${ }_{3}$ ) 205.9 (C-16), 175.1 (C-7), 156.0 (C-9), 145.1 (C-1), 138.4 (C-15), 136.3 (Cipso), 135.3 (C-8), 129.2 (C-13), 128.8, 127.9, and 127.7 (Ph), 121.7 (C-14), 66.3 (C-4), 52.5 (C-6), $44.6\left(\mathrm{CH}_{2} \mathrm{Ph}\right), 44.3$ (C-17), 42.3 (C-5), 34.8 (C10), 33.5 (C-11), 31.5 (C-21), 28.4 (C-3), 28.2 (C.2), 27.7 (C-12). HRMS (ESI) m/z: $[\mathrm{M}+\mathrm{H}]^{+}$calcd for $\mathrm{C}_{25} \mathrm{H}_{26} \mathrm{NO}_{2} 372.1958$, found 372.1960 .

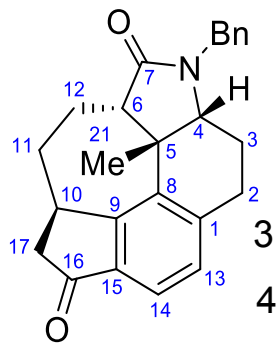

epi-16: ${ }^{1} \mathrm{H}$ NMR (400 MHz, $\left.\mathrm{CDCl}_{3}\right) \delta 7.91(\mathrm{~d}, J=7.6 \mathrm{~Hz}, 1 \mathrm{H}, \mathrm{H}-14)$, 7.34-7.28 (m, 3H, ArH), $7.18(\mathrm{br} \mathrm{d}, J=7.6 \mathrm{~Hz}, 2 \mathrm{H}, \mathrm{ArH}), 7.11(\mathrm{~d}, J=$ $7.6 \mathrm{~Hz}, 1 \mathrm{H}, \mathrm{H}-13), 5.08$ and $3.70\left(2 \mathrm{~d}, J=14.8 \mathrm{~Hz}, 1 \mathrm{H}\right.$ each, $\left.\mathrm{CH}_{2} \mathrm{Ph}\right)$, $3.69(\mathrm{t}, J=7.8 \mathrm{~Hz}, 1 \mathrm{H}, \mathrm{H}-4), 2.94(\mathrm{~m}, 1 \mathrm{H}, \mathrm{H}-10), 2.87$ (br td, $J=14.8$, $4.4 \mathrm{~Hz}, 1 \mathrm{H}, \mathrm{H}-2 \mathrm{ax}$ ), 2.80 (br ddd, $J=17.4,4.1,2.4 \mathrm{~Hz}, 1 \mathrm{H}, \mathrm{H}-17$ ), 2.66 (ddd, $J=14.8,4.8,2.4 \mathrm{~Hz}, 1 \mathrm{H}, \mathrm{H}-2 \mathrm{eq}), 2.62(\mathrm{~m}, 1 \mathrm{H}, \mathrm{H}-6), 2.59(\mathrm{~m}, 1 \mathrm{H}, \mathrm{H}-17), 2.54(\mathrm{~m}$, $1 \mathrm{H}, \mathrm{H}-12), 2.32(\mathrm{~m}, 1 \mathrm{H}, \mathrm{H}-3), 2.25$ (m, 1H, H-11), 1.90 (ddd, J = 13.8, 12.6, 4.4, $1.4 \mathrm{~Hz}$, 1H, $\mathrm{H}-11$ ), 1.78 (dddd, $J=12.6,12.6,12.6,4.8 \mathrm{~Hz}, 1 \mathrm{H}, \mathrm{H}-12), 1.34(\mathrm{~s}, 3 \mathrm{H}, \mathrm{H}-21), 1.29$ 
(m, 1H, H-3); ${ }^{13} \mathrm{C}$ NMR (100 MHz, $\left.\mathrm{CDCl}_{3}\right) \delta 198.1$ (C-16), 174.3 (C-7), 145.8 (C-9), 141.9 (C-1), 136.2 (C-15), 136.0 (Cipso), 130.7 (C-8), 128.7, 128.1, and 127.6 (C Ar), 126.1 (C-14), 126.0 (C-13), 61.1 (C-4), 48.4 (C-6), $43.7\left(\mathrm{CH}_{2} \mathrm{Ph}\right), 39.3$ (C-5), 39.0 (C17), 31.6 (C-10), 31.1 (C-11), 27.6 (C-2 and C-21), 26.9 (C-3), 24.7 (C-12). HRMS (ESI) $\mathrm{m} / \mathrm{z}:[\mathrm{M}+\mathrm{H}]^{+}$calcd for $\mathrm{C}_{25} \mathrm{H}_{26} \mathrm{NO}_{2} 372.1958$, found 372.1963 .

\section{Pentacyclic lactam 17}

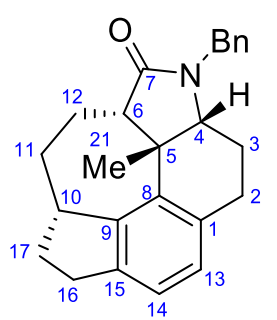

To a solution of ketone $16(65 \mathrm{mg}, 0.17 \mathrm{mmol})$ in $\mathrm{MeOH}(8 \mathrm{~mL})$ and acetic acid $(0.8 \mathrm{~mL})$ was added $\mathrm{Pd} / \mathrm{C} 10 \%(93 \mathrm{mg})$. The reaction was stirred overnight at room temperature under a $\mathrm{H}_{2}$ atmosphere (1 atm). The mixture was filtered on a celite ${ }^{\circledR}$ pad and concentrated to give lactam 17 as a colorless oil (56 mg, 90\%): ${ }^{1} \mathrm{H}$ NMR (400 MHz, $\left.\mathrm{CDCl}_{3}\right)$

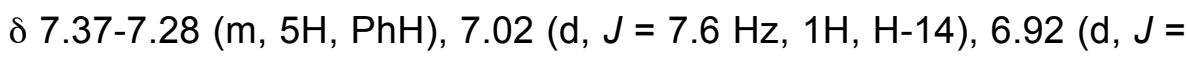
$7.6 \mathrm{~Hz}, 1 \mathrm{H}, \mathrm{H}-13), 5.12$ and $4.04\left(2 \mathrm{~d}, J=14.8 \mathrm{~Hz}, 1 \mathrm{H}\right.$ each, $\left.\mathrm{CH}_{2} \mathrm{Ph}\right), 3.36(\mathrm{br} \mathrm{q}, J=8.0$ $\mathrm{Hz}, 1 \mathrm{H}, \mathrm{H}-10), 3.28$ (br dd, J = 11.0, 4.2 Hz, 1H, H-4), 2.97-2.80 (m, 2H, H-16), 2.65 (br $\mathrm{dt}, J=15.4,4.0 \mathrm{~Hz}, 1 \mathrm{H}, \mathrm{H}-2 \mathrm{eq}), 2.51(\mathrm{~m}, 2 \mathrm{H}, \mathrm{H}-6$ and H-2ax), $2.31(\mathrm{~m}, 1 \mathrm{H}, \mathrm{H}-17), 2.20$ (m, 1H, H-3), $2.11(\mathrm{~m}, 1 \mathrm{H}, \mathrm{H}-12), 1.99$ (dddd, $J=13.5,13.5,7.2,7.2,1 \mathrm{H}, \mathrm{H}-11), 1.79$ (m, 1H, H-17), $1.45(\mathrm{~m}, 1 \mathrm{H}, \mathrm{H}-11), 1.34(\mathrm{~s}, 3 \mathrm{H}, \mathrm{H}-21), 1.20(\mathrm{~m}, 2 \mathrm{H}, \mathrm{H}-12$ and $\mathrm{H}-3) ;{ }^{13} \mathrm{C}$ NMR (100 MHz, CDCl ${ }_{3}$ ) $\delta 176.0$ (C-7), 144.0 (C-9), 141.9 (C-1), 136.6 (C-15), 136.1 (Cipso), 135.3 (C-8), 128.7, 127.9, and 127.5 (C Ar), 127.3 (C-13), 122.7 (C-14), 66.0 (C-4), 53.1 (C-6), $44.3\left(\mathrm{CH}_{2} \mathrm{Ph}\right), 42.2$ (C-5), 41.4 (C-10), 31.4 (C-17), $31.0(\mathrm{C}-21), 30.6$ (C-16), 30.1 (C-11), 28.1 (C-3), 27.2 (C-2), 26.9 (C-12). HRMS (ESI) m/z: [M+H] calcd for $\mathrm{C}_{25} \mathrm{H}_{28} \mathrm{NO} 358.2165$, found 358.2171. 
Biosynthetic numbering

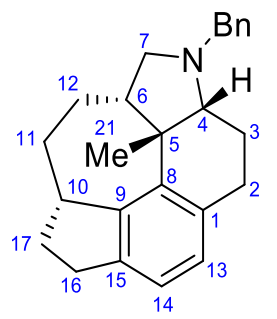

To a solution of lactam $17(56 \mathrm{mg}, 0.16 \mathrm{mmol})$ in ether $(7 \mathrm{~mL})$ was added $\mathrm{LiAlH}_{4}(59$ $\mathrm{mg}, 1.57 \mathrm{mmol}, 10$ equiv) and the mixture was stirred for $24 \mathrm{~h}$ at $40^{\circ} \mathrm{C}$. The reaction was quenched with $\mathrm{Na}_{2} \mathrm{SO}_{4} \cdot 10 \mathrm{H}_{2} \mathrm{O}(700 \mathrm{mg})$, stirred for $10 \mathrm{~min}$, filtered and washed several times with ether. The ethereal layers were dried and concentrated to afford amine 18 as a colorless oil (47 mg, 89\%): ${ }^{1} \mathrm{H}$ NMR (400 MHz, $\left.\mathrm{CDCl}_{3}\right) \delta$ 7.31-7.28 (m, $3 \mathrm{H}, \mathrm{ArH}), 7.24-7.19(\mathrm{~m}, 1 \mathrm{H}, \mathrm{ArH}), 6.99$ (br d, J = 7.6 Hz, 1H, H-14), 6.95 (br d, J = 7.6 $\mathrm{Hz}, 1 \mathrm{H}, \mathrm{H}-13$ ), 4.03 and $3.25\left(2 \mathrm{~d}, J=13.2 \mathrm{~Hz}, 1 \mathrm{H}\right.$ each, $\mathrm{CH}_{2} \mathrm{Ph}$ ), 3.42 (dddd, $J=13.0$, 13.0, 8.0, 8.0 Hz, 1H, H-10), $2.92(\mathrm{~m}, 1 \mathrm{H}, \mathrm{H}-2), 2.88$ and $2.79(2 \mathrm{~m}, 1 \mathrm{H}$ each, $\mathrm{H}-16)$, $2.56(\mathrm{~m}, 1 \mathrm{H}, \mathrm{H}-4), 2.53\left(\mathrm{~m}, 3 \mathrm{H}, \mathrm{H}-2\right.$ and $\left.7-\mathrm{CH}_{2}\right), 2.27$ (dddd, $J=13.0,8.0,8.0,7.2 \mathrm{~Hz}$, $1 \mathrm{H}, \mathrm{H}-17), 2.04(\mathrm{~m}, 1 \mathrm{H}, \mathrm{H}-6), 2.00(\mathrm{~m}, 1 \mathrm{H}, \mathrm{H}-3), 1.83(\mathrm{~m}, 1 \mathrm{H}, \mathrm{H}-11), 1.71(\mathrm{~m}, 1 \mathrm{H}, \mathrm{H}-$ 17), $1.68(\mathrm{~m}, 1 \mathrm{H}, \mathrm{H}-3), 1.49(\mathrm{~m}, 1 \mathrm{H}, \mathrm{H}-11), 1.39\left(\mathrm{~m}, 2 \mathrm{H}, \mathrm{CH}_{2}-12\right), 1.34(\mathrm{~s}, 3 \mathrm{H}, \mathrm{H}-21)$; ${ }^{13} \mathrm{C}$ NMR (100 MHz, CDCl $\left.{ }_{3}\right) \delta 144.3(\mathrm{C}-9), 141.5$ (C-1), $140.2\left(\mathrm{C}_{i p s o}\right), 138.4(\mathrm{C}-15)$, 135.7 (C-8), $128.4(\mathrm{Ph}), 128.0(\mathrm{Ph}), 127.2(\mathrm{C}-13), 126.5(\mathrm{Ph}), 121.8(\mathrm{C}-14), 72.3(\mathrm{C}-4)$, 59.6 (C-7), $57.8\left(\mathrm{CH}_{2} \mathrm{Ph}\right), 47.7$ (C-5), 46.8 (C-6), 42.3 (C-10), 32.5 (C-17), 30.8 (C-16), 30.1 (C-11), 29.9 (C-12), 29.3 (C-21), 26.4 (C-2), 24.7 (C-3). HRMS (ESI) m/z: [M+H] ${ }^{+}$ calcd for $\mathrm{C}_{25} \mathrm{H}_{30} \mathrm{~N} 344.2373$, found 344.2373. 


\section{Preliminary studies in search of a route to construct the piperidine B ring}

Achieving a functionalization at $\mathbf{1 6}$ toward the elaboration of the remaining ring en route to daphenylline proved to be non-straightforward process. Bromide S-1 was prepared in moderate yield and submitted to a Keck radical allylation. Attempts using AIBN at 80 ${ }^{\circ} \mathrm{C}$ led to the corresponding elimination process giving alkene S-2. Reaction conditions with a low temperature $\left(\mathrm{Et}_{3} \mathrm{~B}, \mathrm{O}_{2}\right)$ resulted in the isolation of a negligible amount of the allylated compound.

The challenging radical cyclization cascade from S-3, obtained by $\mathrm{N}$-debenzylation of 18 and $N$-propargylation of the secondary amine intermediate, failed to produce the desired pentacyclic ring system of daphenylline. Under radical conditions, a benzenethiol addition reaction using S-3 as a substrate generated a mixture in which formamide S-4 was the only compound identified by NMR and MS.

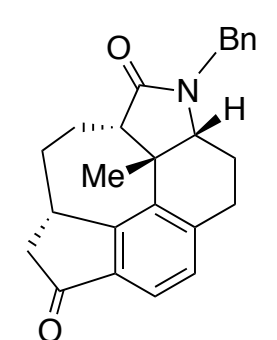

16

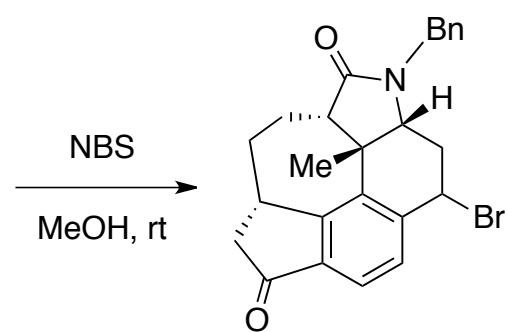

S-1

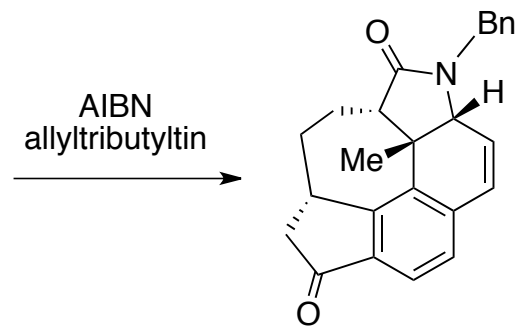

S-2

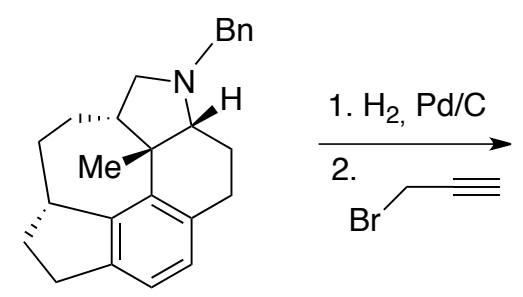

18<smiles>C#CCN1C[C@@H]2CC[C@@H]3CCc4ccc5c(c4[C@@]32C)[C@@]1(C)CC5</smiles>

S-3

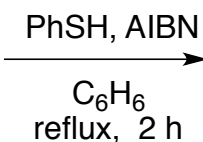<smiles>C[C@]12[C@H]3CC[C@H]4CCc5ccc(c(c54)[C@@]31C)CC[C@H]2NC=O</smiles>

S-4 


\section{Bromide S-1}

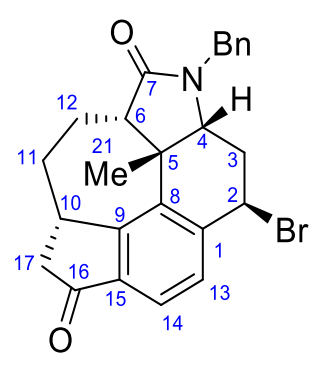

A mixture of ketone 16 (42 $\mathrm{mg}, 0.15 \mathrm{mmol})$, NBS (29 mg, 0.16 mmol, 1.1 equiv) and AIBN (7 mg, $0.04 \mathrm{mmol}, 0.3$ equiv) in benzene $(6 \mathrm{~mL})$ was refluxed for $3 \mathrm{~h}$ and then cooled to room temperature. The reaction mixture was extracted with EtOAc $(3 \times 5$ $\mathrm{mL}$ ) and the organic extracts were dried, concentrated, and purified by chromatography (Hexane/EtOAc $95: 5$ to $1: 1$ ) to afford bromide S-1 as a pale brownish oil (14 mg, 28\%, dr 5:1): ${ }^{1} \mathrm{H}$ NMR $\left(400 \mathrm{MHz}, \mathrm{CDCl}_{3}\right) \delta$ $7.62(\mathrm{~d}, J=8.0 \mathrm{~Hz}, 1 \mathrm{H}, \mathrm{H}-14), 7.40-7.35(\mathrm{~m}, 6 \mathrm{H}, \mathrm{ArH}$ and $\mathrm{H}-13), 5.35(\mathrm{br} \mathrm{t}, J=2.8 \mathrm{~Hz}$, $1 \mathrm{H}, \mathrm{H}-2), 4.97$ and $4.24\left(2 \mathrm{~d}, J=14.8 \mathrm{~Hz}, 1 \mathrm{H}\right.$ each, $\left.\mathrm{CH}_{2} \mathrm{Ph}\right), 4.02(\mathrm{dd}, J=11.8,4.6 \mathrm{~Hz}$, $1 \mathrm{H}, \mathrm{H}-4), 3.58(\mathrm{~m}, 1 \mathrm{H}, \mathrm{H}-10), 2.97$ (dd, J = 19.0, 7.4 Hz, 1H, H-17), $2.65(\mathrm{~m}, 1 \mathrm{H}, \mathrm{H}-3)$, $2.62(\mathrm{~m}, 1 \mathrm{H}, \mathrm{H}-6), 2.34(\mathrm{~m}, 1 \mathrm{H}, \mathrm{H}-11), 2.32(\mathrm{~m}, 1 \mathrm{H}, \mathrm{H}-17), 2.22(\mathrm{~m}, 1 \mathrm{H}, \mathrm{H}-12), 1.54(\mathrm{~m}$, $1 \mathrm{H}, \mathrm{H}-3), 1.48(\mathrm{~s}, 1 \mathrm{H}, \mathrm{H}-21), 1.37(\mathrm{~m}, 1 \mathrm{H}, \mathrm{H}-12), 1.32(\mathrm{~m}, 1 \mathrm{H}, \mathrm{H}-11) ;{ }^{13} \mathrm{C}$ NMR $(100$ $\mathrm{MHz}, \mathrm{CDCl}_{3}$ ) $\delta 205.4$ (C-16), 174.9 (C-7), 156.2 (C-9), 143.0 (C-1), 138.1 (C-15), 137.1 (C-8), 136.2 ( ( $\left._{\text {ipso }}\right), 129.2$ (C-13), 128.9, 128.0, and 127.9 (C Ar), 122.5 (C-14), 62.4 (C-4), 52.6 (C-6), 46.1 (C-2), 45.1 ( $\mathrm{CH}_{2} \mathrm{Ph}$ ), 44.0 (C-17), 41.8 (C-5), 36.3 (C-3), 34.7 (C-10), 33.6 (C-11), 31.2 (C-21), 27.7 (C-12). HRMS (ESI) m/z: $[\mathrm{M}+\mathrm{H}]^{+}$calcd for $\mathrm{C}_{25} \mathrm{H}_{25} \mathrm{BrNO}_{2}$ 450.1063, found 450.1055.

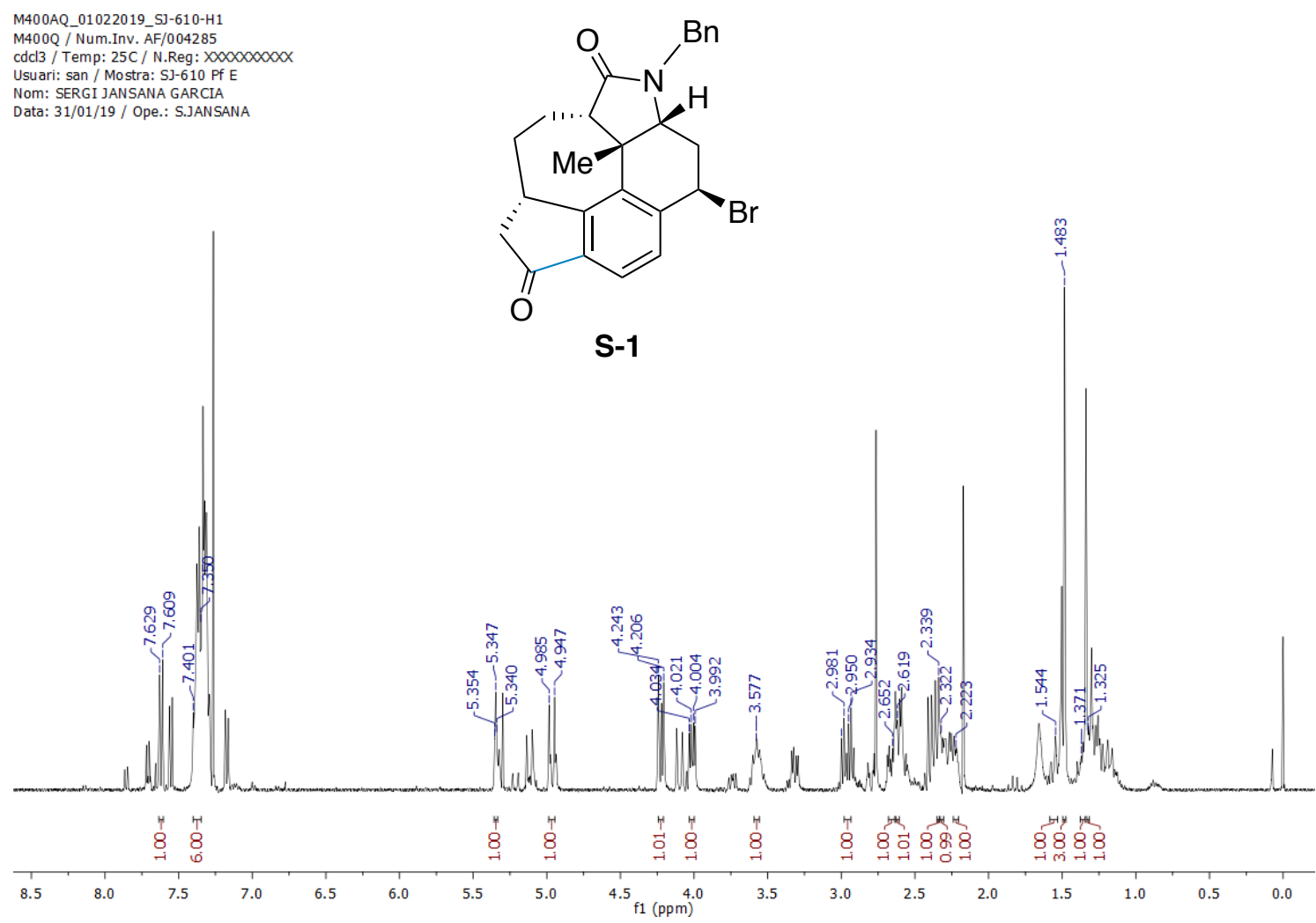




\section{Alkene S-2}

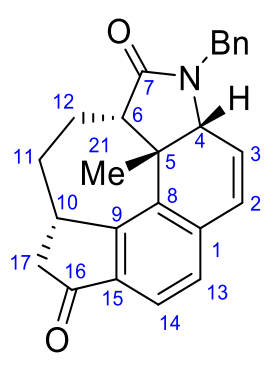

Bromide S-1 (8 $\mathrm{mg}, 0.02 \mathrm{mmol})$ was dissolved in benzene $(0.3 \mathrm{~mL})$. Allyltributyltin ( $11 \mu \mathrm{L}, 0.04 \mathrm{mmol}, 2.4$ equiv) and AIBN (1 mg, $4 \times 10^{-3}$ mmol, 0.2 equiv) were added and the mixture was refluxed for $1 \mathrm{~h}$ in a sealed tube. The solution was cooled to room temperature, followed by addition of DBU ( $6 \mu \mathrm{L}, 0.04 \mathrm{mmol}, 2$ equiv). After solvent evaporation and purification by chromatography (Hexane/EtOAc 9:1 to $1: 1)$ alkene S-2 was obtained as a colorless oil $(5 \mathrm{mg}, 84 \%):{ }^{1} \mathrm{H}$ NMR $(400 \mathrm{MHz}$, $\left.\mathrm{CDCl}_{3}\right) \delta 7.61(\mathrm{~d}, J=7.6 \mathrm{~Hz}, 1 \mathrm{H}), 7.33-7.27(\mathrm{~m}, 3 \mathrm{H}), 7.17(\mathrm{~m}, 2 \mathrm{H}), 7.13(\mathrm{~d}, J=7.6 \mathrm{~Hz}$, $1 \mathrm{H}), 6.74(\mathrm{~d}, J=9.6 \mathrm{~Hz}, 1 \mathrm{H}), 5.91(\mathrm{dd}, J=9.6,5.8 \mathrm{~Hz}, 1 \mathrm{H}), 4.94$ and 3.88 (two d, $J=$ $14.8 \mathrm{~Hz}, 1 \mathrm{H}$ each), $3.68(\mathrm{~m}, 1 \mathrm{H}), 3.31(\mathrm{~m}, 1 \mathrm{H}), 3.00(\mathrm{dd}, J=19.2,7.6 \mathrm{~Hz}, 1 \mathrm{H}), 2.70(\mathrm{br}$ $\mathrm{t}, J=4.0 \mathrm{~Hz}, 1 \mathrm{H}), 2.62(\mathrm{~m}, 1 \mathrm{H}), 2.34(\mathrm{dd}, J=19.2,4.4 \mathrm{~Hz}, 1 \mathrm{H}), 2.09(\mathrm{~m}, 1 \mathrm{H}), 1.77(\mathrm{~m}$, 1H), $1.64(\mathrm{~m}, 1 \mathrm{H}), 1.36(\mathrm{~s}, 3 \mathrm{H}) ;{ }^{13} \mathrm{C}$ NMR (100 MHz, $\left.\mathrm{CDCl}_{3}\right) \delta 205.3,157.6,142.2$, $138.1,137.4,136.2,133.1,128.7,128.7,127.8,127.5,122.7,122.6,60.8,52.3,48.0$, $44.7,43.8,40.6,31.2,25.6,21.8$. 

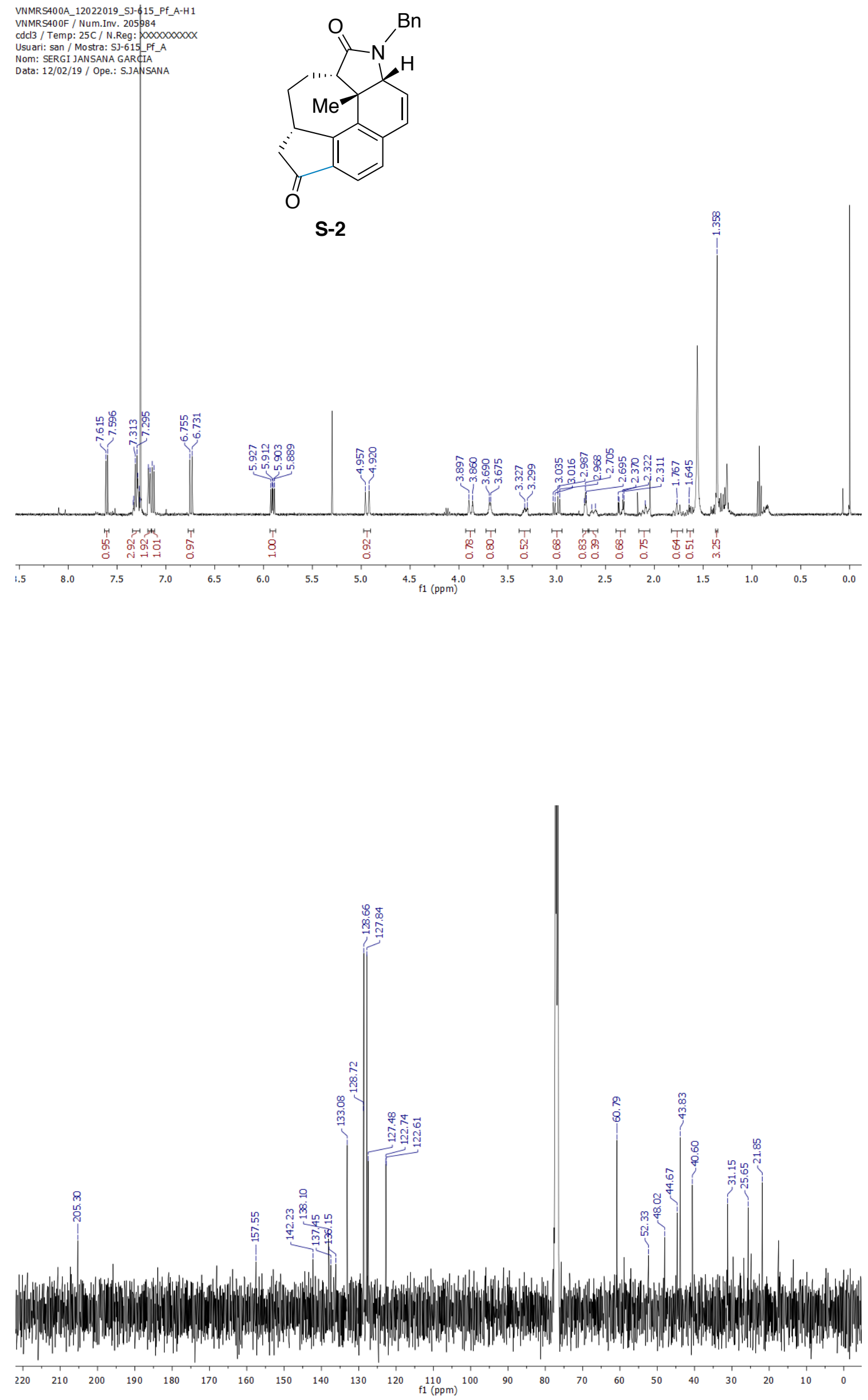


\section{Amine S-3}

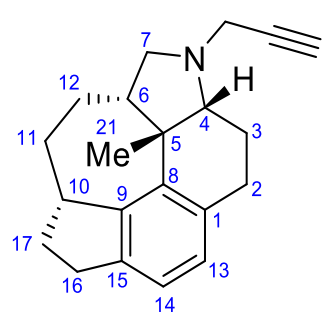

Amine 18 (47 mg, $0.14 \mathrm{mmol}, 1.0$ equiv) was dissolved in $\mathrm{MeOH}$ $(1.5 \mathrm{~mL})$, and $\mathrm{Pd} / \mathrm{C} 10 \%$ (29 mg) was added. The resulting suspension was stirred under $\mathrm{H}_{2}$ atmosphere $(1 \mathrm{~atm})$ at room temperature. After $24 \mathrm{~h}$, the reaction mixture was filtered through celite $^{\circledR}$ pad and concentrated to afford the corresponding secondary amine as a brown oil ( $30 \mathrm{mg}, 0.12 \mathrm{mmol})$. To a solution of the above secondary amine in acetonitrile $(0.6 \mathrm{~mL})$ was added $\mathrm{K}_{2} \mathrm{CO}_{3}(33 \mathrm{mg}, 0.24$ mmol, 2.0 equiv) and propargyl bromide ( $80 \%$ in toluene, $20 \mu \mathrm{l}, 0.18 \mathrm{mmol}, 1.5$ equiv). The reaction mixture was stirred $22 \mathrm{~h}$ at room temperature, quenched with water and extracted with EtOAc $(3 \times 10 \mathrm{~mL})$. The combined organics were washed with brine, filtered, concentrated and purified by chromatography $\left(\mathrm{CH}_{2} \mathrm{Cl}_{2} / \mathrm{MeOH} 1: 0\right.$ to $\left.9: 1\right)$ to afford amine S-3 as a brownish oil (22 mg, 65\% over 2 steps): ${ }^{1} \mathrm{H}$ NMR (400 MHz, $\left.\mathrm{CDCl}_{3}\right) \delta 6.96(\mathrm{br} \mathrm{d}, J=7.6 \mathrm{~Hz}, 1 \mathrm{H}, \mathrm{H}-14), 6.92(\mathrm{br} \mathrm{d}, J=7.6 \mathrm{~Hz}, 1 \mathrm{H}, \mathrm{H}-13), 3.51$ and $3.48\left(2 \mathrm{dd}, J=15.4,2.4 \mathrm{~Hz}, 1 \mathrm{H}\right.$ each, $\mathrm{NCH}_{2}$ ), $3.43(\mathrm{~m}, 1 \mathrm{H}, \mathrm{H}-10), 2.99$ (dd, $J=9.0,8.0$ $\mathrm{Hz}, 1 \mathrm{H}, \mathrm{H}-7), 2.89(\mathrm{~m}, 1 \mathrm{H}, \mathrm{H}-16), 2.81-2.73(\mathrm{~m}, 2 \mathrm{H}, \mathrm{H}-2$ and $\mathrm{H}-16), 2.70(\mathrm{dd}, J=6.8$, $4.0 \mathrm{~Hz}, 1 \mathrm{H}, \mathrm{H}-4$ ), 2.59 (dd, $J=9.0,4.4 \mathrm{~Hz}, 1 \mathrm{H}, \mathrm{H}-7$ ), 2.53 (ddd, $J=11.6,6.8,4.4 \mathrm{~Hz}$, $1 \mathrm{H}, \mathrm{H}-2), 2.28(\mathrm{~m}, 1 \mathrm{H}, \mathrm{H}-17), 2.20(\mathrm{t}, \mathrm{J}=2.4 \mathrm{~Hz}, \equiv \mathrm{CH}), 2.09(\mathrm{~m}, 1 \mathrm{H}, \mathrm{H}-6), 2.00(\mathrm{~m}, 1 \mathrm{H}$, $\mathrm{H}-3), 1.88(\mathrm{~m}, 1 \mathrm{H}, \mathrm{H}-11), 1.70(\mathrm{~m}, 1 \mathrm{H}, \mathrm{H}-17), 1.61(\mathrm{~m}, 1 \mathrm{H}, \mathrm{H}-12), 1.52(\mathrm{~m}, 1 \mathrm{H}, \mathrm{H}-3)$, $1.42(\mathrm{~m}, 2 \mathrm{H}, \mathrm{H}-11$ and $\mathrm{H}-12), 1.33(\mathrm{~s}, 3 \mathrm{H}, \mathrm{H}-21) ;{ }^{13} \mathrm{C}$ NMR $\left(100 \mathrm{MHz}, \mathrm{CDCl}_{3}\right) \delta 144.1$ (C-9), 141.6 (C-1), 138.1 (C-15), 135.2 (C-8), 127.4 (C-13), 121.9 (C-14), 78.8 (C三CH), $72.7(\mathrm{CH} \equiv \mathrm{C}), 69.0$ (C-4), 58.1 (C-7), 47.6 (C-5), 46.9 (C-6), 42.2 (C-10), $40.3\left(\mathrm{CH}_{2}-\mathrm{C} \equiv\right)$, 32.6 (C-17), 30.8 (C-16), 30.2 (C-11), 30.1 (C-12), 29.1 (C-21), 26.1 (C-2), 24.4 (C-3). HRMS (ESI) $\mathrm{m} / \mathrm{z}:[\mathrm{M}+\mathrm{H}]^{+}$calcd for $\mathrm{C}_{21} \mathrm{H}_{26} \mathrm{~N} 292.206$, found 292.2061.

\section{Formamide S-4}

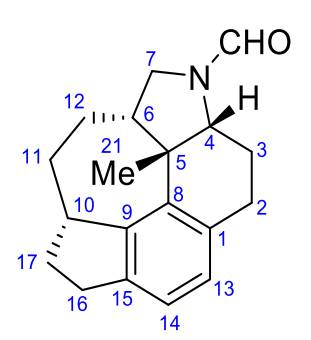

To a refluxing solution of amine $\mathbf{S - 3}(22 \mathrm{mg}, 0.08 \mathrm{mmol})$ in tertbutanol (12 mL) was added AIBN (25 mg, $0.15 \mathrm{mmol}, 2.0$ equiv) and thiophenol (16 $\mu \mathrm{L}, 0.15 \mathrm{mmol}, 2.0$ equiv) in toluene ( $1 \mathrm{~mL}$ ) over $20 \mathrm{~h}$ via a syringe pump. Once the addition was complete, the reaction was kept at reflux for $2 \mathrm{~h}$, cooled to room temperature, and concentrated. The residue was purified by chromatography $\left(\mathrm{CH}_{2} \mathrm{Cl}_{2} / \mathrm{MeOH}\right.$ 1:0 to 9:1): ${ }^{1} \mathrm{H}$ NMR (400 MHz, $\left.\mathrm{CDCl}_{3}\right) \delta 8.25(\mathrm{~s}, 1 \mathrm{H}), 7.02(\mathrm{br} \mathrm{d}, J=7.6$ $\mathrm{Hz}, 1 \mathrm{H}), 6.90$ (br d, $J=7.6 \mathrm{~Hz}), 3.94(\mathrm{~m}, 1 \mathrm{H}), 3.60$ (dd, $J=8.8,7.4 \mathrm{~Hz}, 1 \mathrm{H}), 3.42(\mathrm{~m}$, $1 \mathrm{H}), 1.18$ and $1.16\left(2 \mathrm{~s}, 3 \mathrm{H}\right.$, two rotamers); HRMS (ESI) $\mathrm{m} / \mathrm{z}$ : $[\mathrm{M}+\mathrm{H}]^{+}$calcd for $\mathrm{C}_{19} \mathrm{H}_{24} \mathrm{NO}$ 282.1852, found 282.1853 . 
VNMRS400A_26042019_SJ-632_Pf_A-H1_rep_03_27_23 VNMRS400F / Num.Inv. 205984

Juri: san / Mostra: SJ 632 $P$ Cf

IOm: SERGI JANSANA G32_Pf A

Data: 26/04/19 / Ope.: S.JANSANA

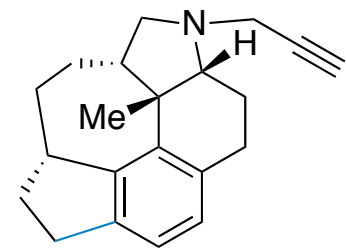

S-3
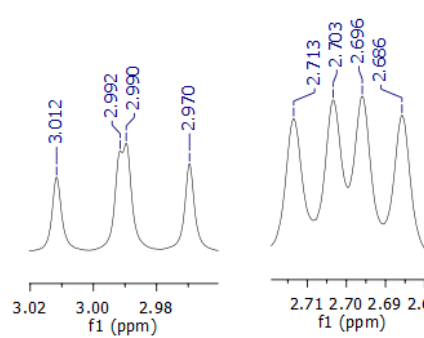

2.712 .702 .69
$f 1(\mathrm{ppm})$
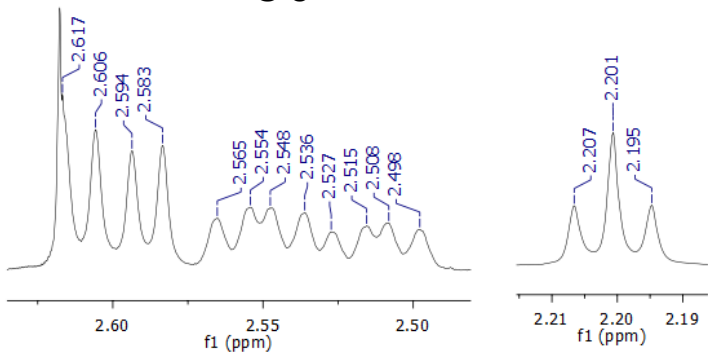

$2.21 \underset{\mathrm{f} 1(\mathrm{ppm})}{2.20} 2.19$
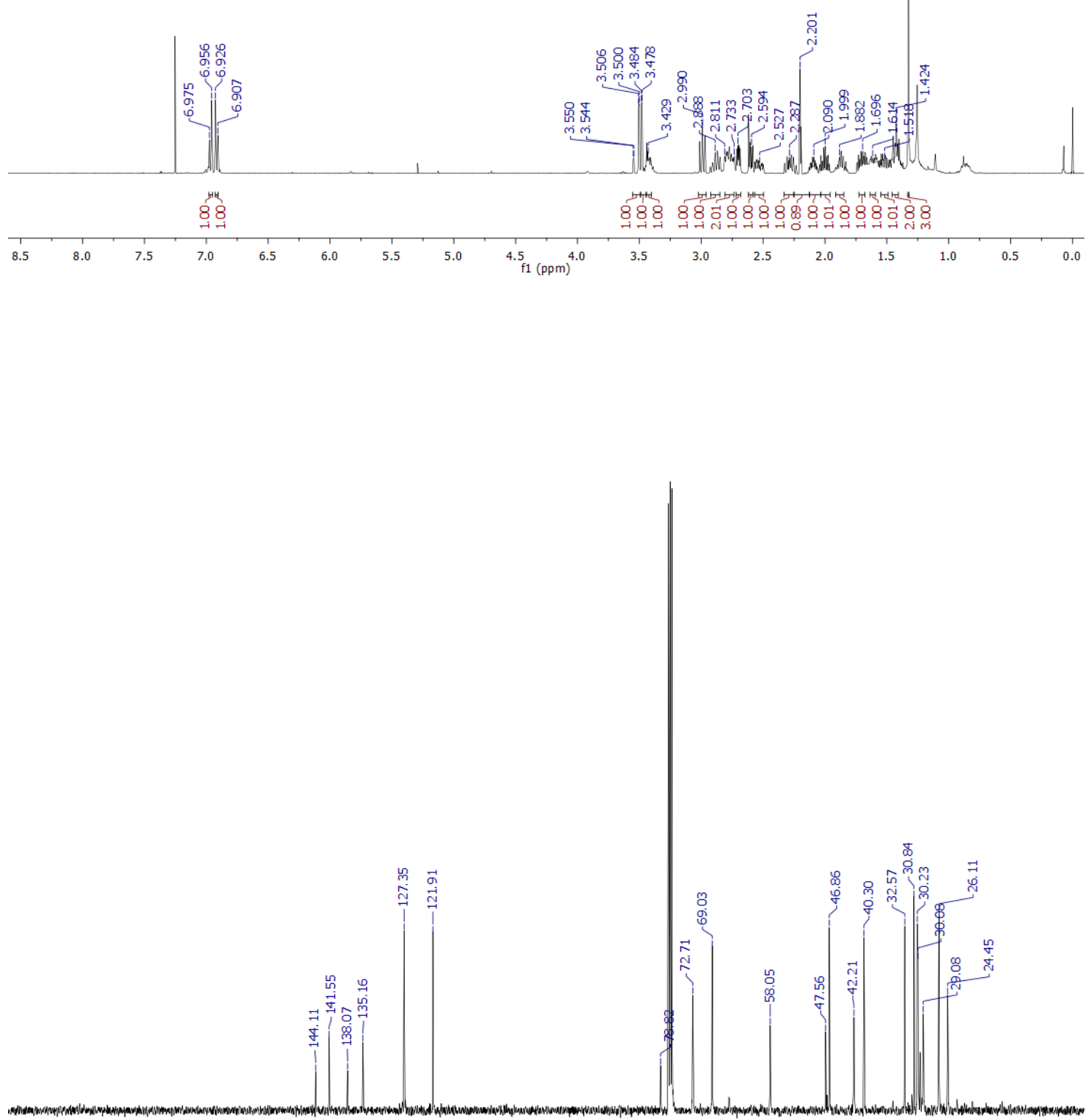

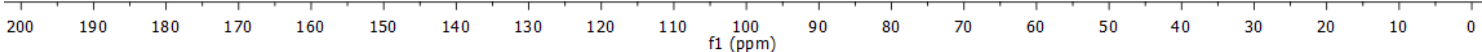


M400AFF_02052017_SJ-292-H1

M400F/ Num.Inv. 1009191

Usuari: san / Mostra: SJ-292 Pf A

Dom: SERGI JANSANA GARCIA
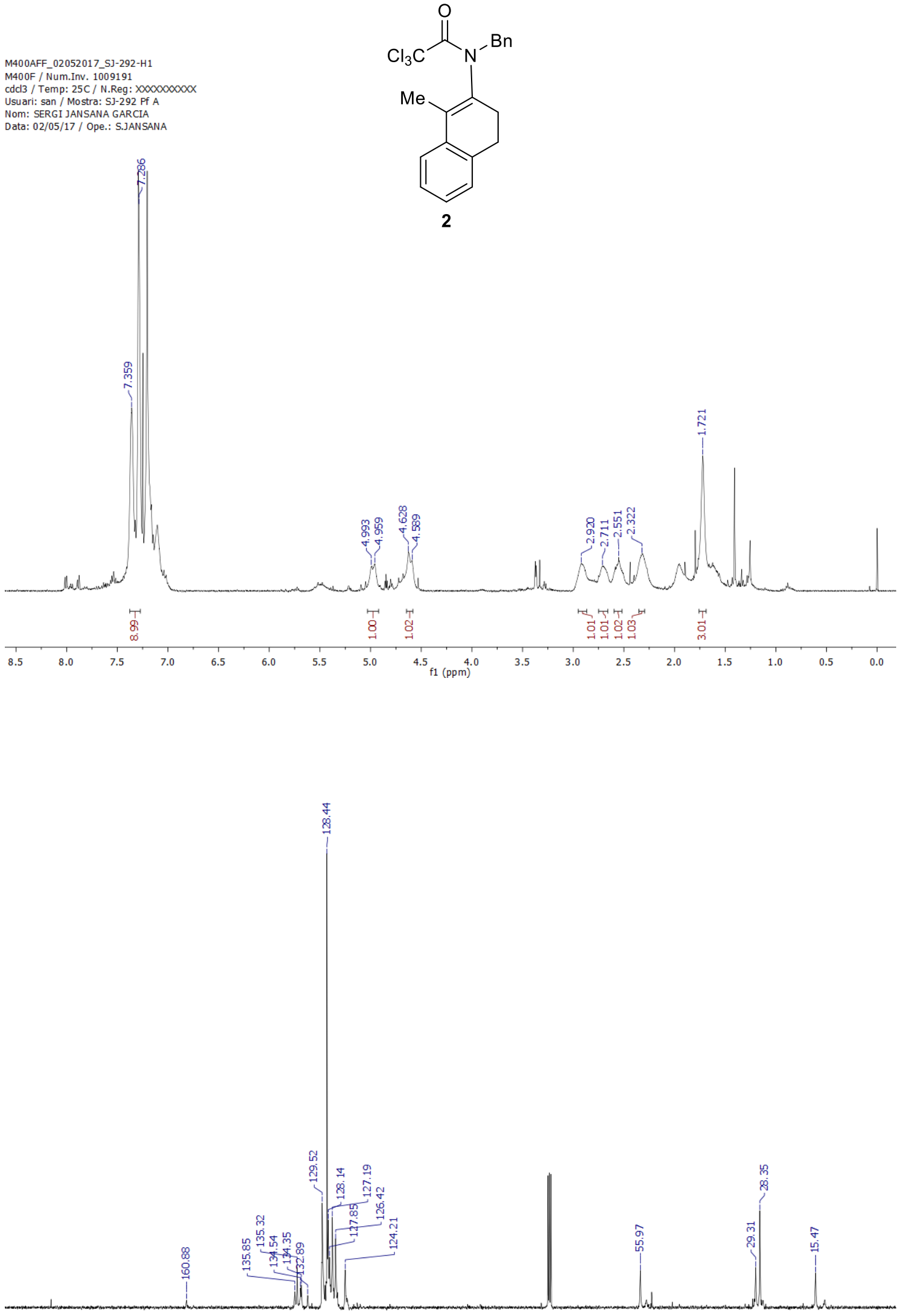

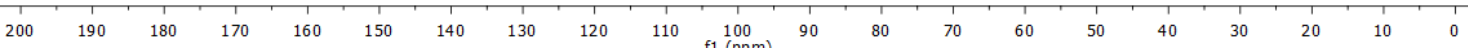


VNMRS400A_09052017_SJ-296_Pf_B-H1

VNMRS400F / Num.Inv. 205984

Usuari: san / Mostra: SJ-296_Pf_B

Data: 09/05/17 / Ope.: S.JANSANA
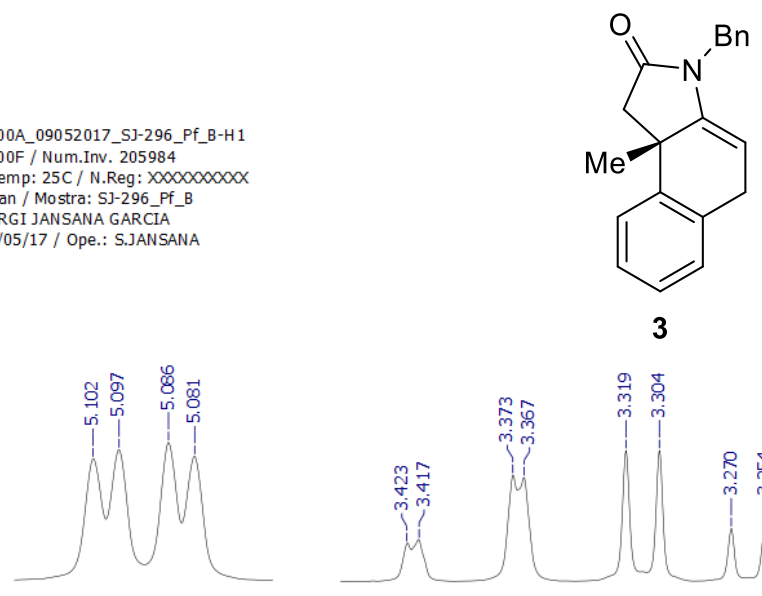

$\begin{array}{llllll}5.11 & 5.10 & 5.09 & 5.08 & 5.07\end{array}$

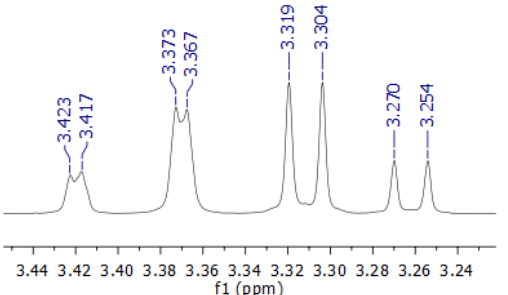

$\mathrm{f} 1$ (ppm)
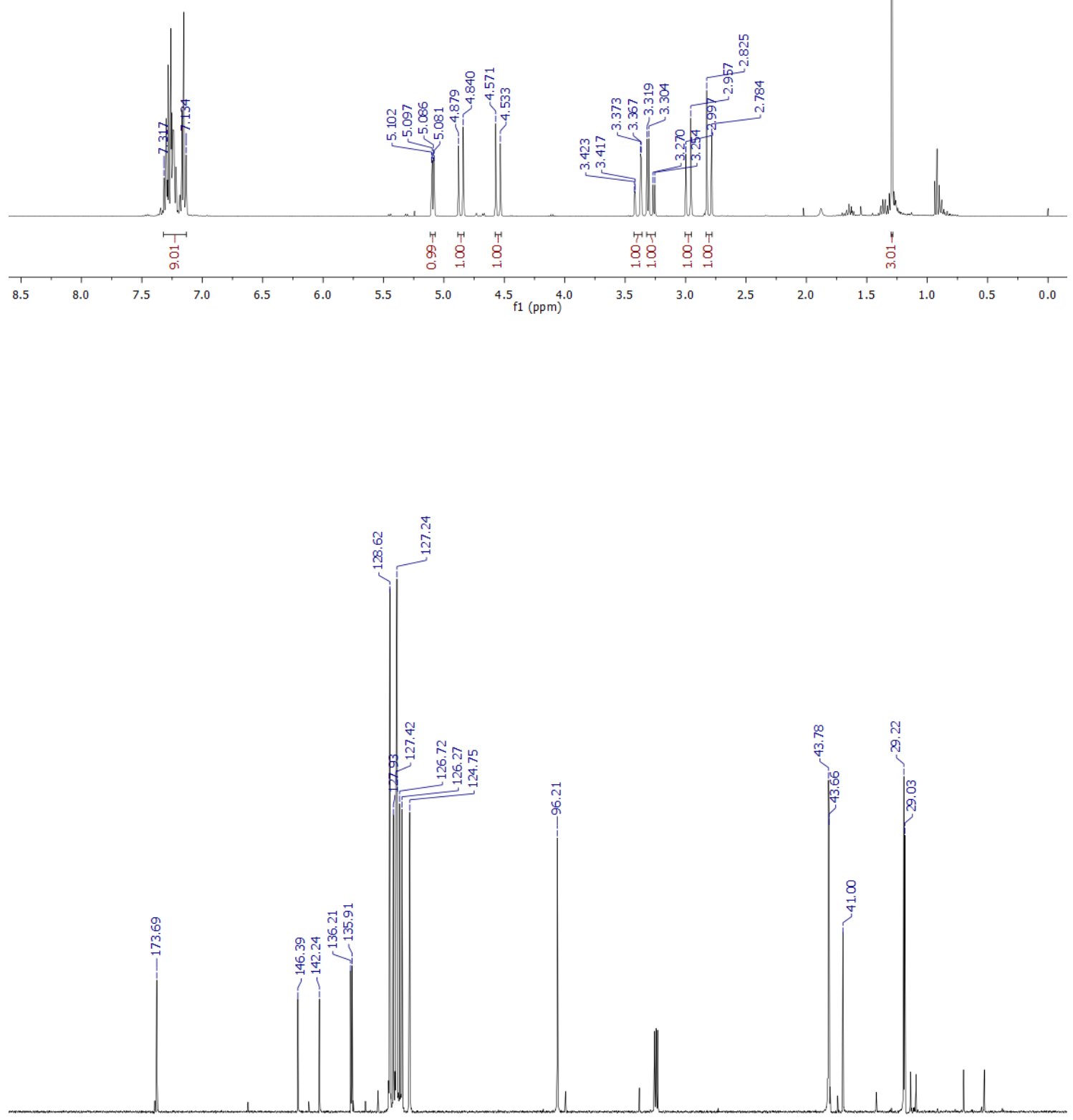

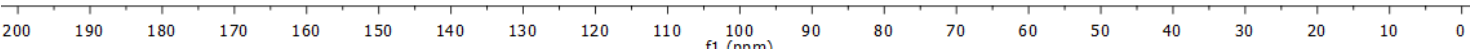


M400AFF_26012017_SJ-243_Pf_B-H1 M400F / Num.Inv. 1009191

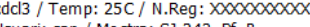
Usuari: san / Mostra: SJ-243_Pf_B Data: 26/01/17 / Ope.: S.JANSANA
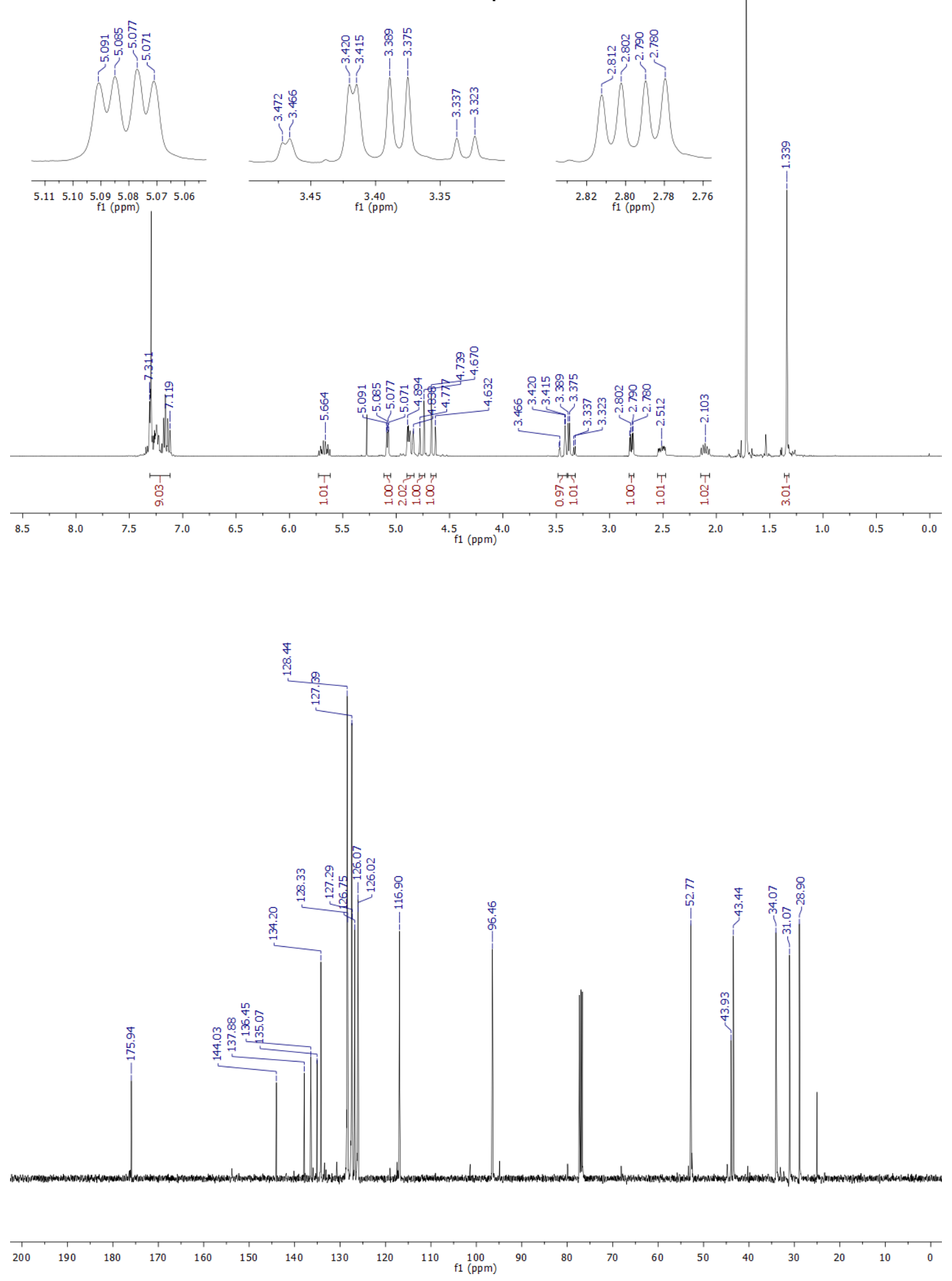

S25 
M00AFF_03022017_SJ-244-46_Pf_C-H1

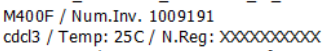

Usuari: san / Mostra: SJ-244-46_Pf_C
Nom: SERGI JANSANA GARCIA<smiles>C=CC[C@H]1C(=O)N(Cc2ccccc2)[C@@H]2CCc3ccccc3[C@]12C</smiles>
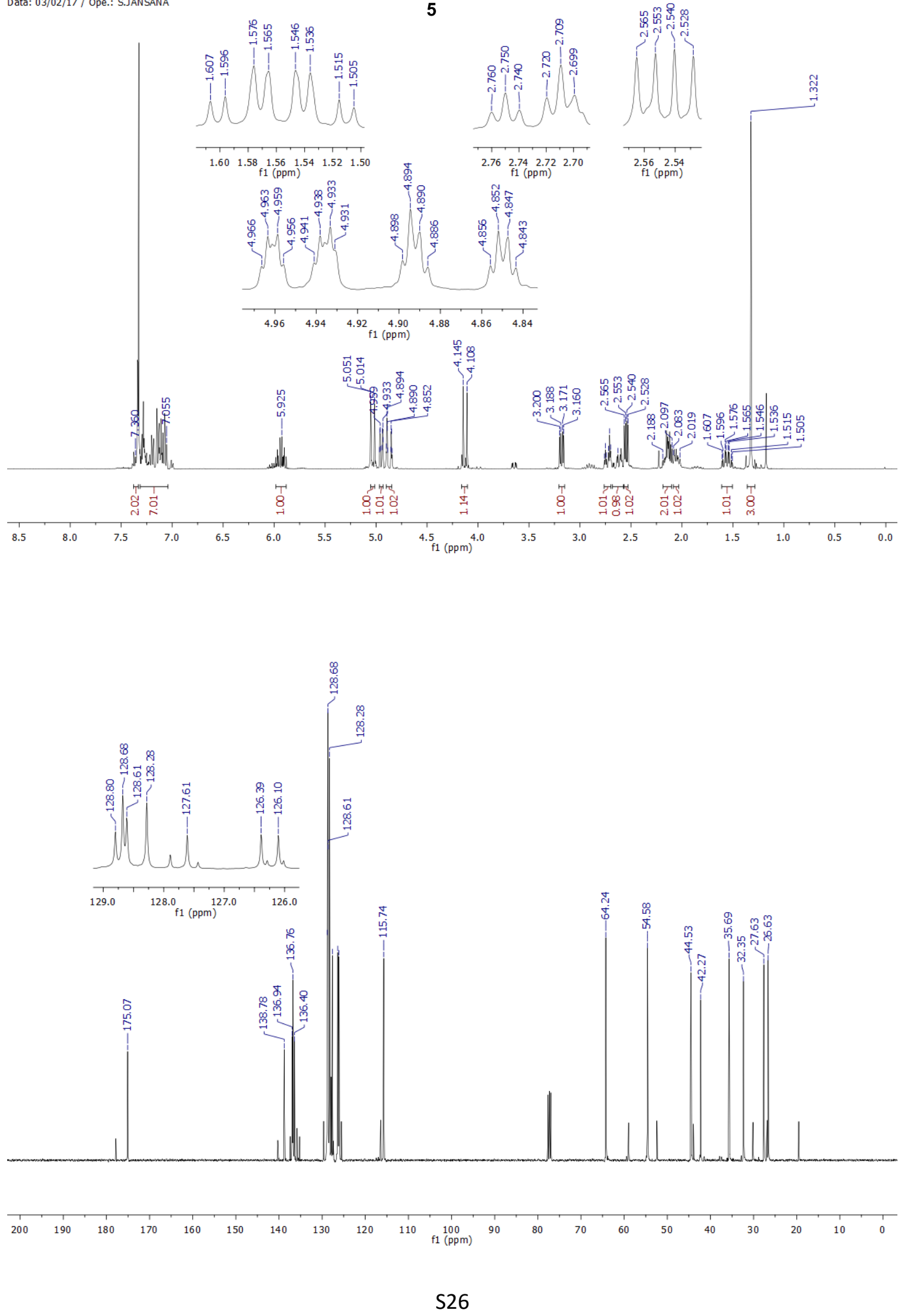
VNMRS400A_10022017_SJ-248_C_Pf_A-H1 NMRS400F / Num.Inv. 205984

(d)

Data: 10/02/17/OPA.: SJANSANA<smiles>[M][C@]1(CCCO)C(=O)N(Cc2ccccc2)[C@]2(C)CCc3ccccc3[C@H]12</smiles>
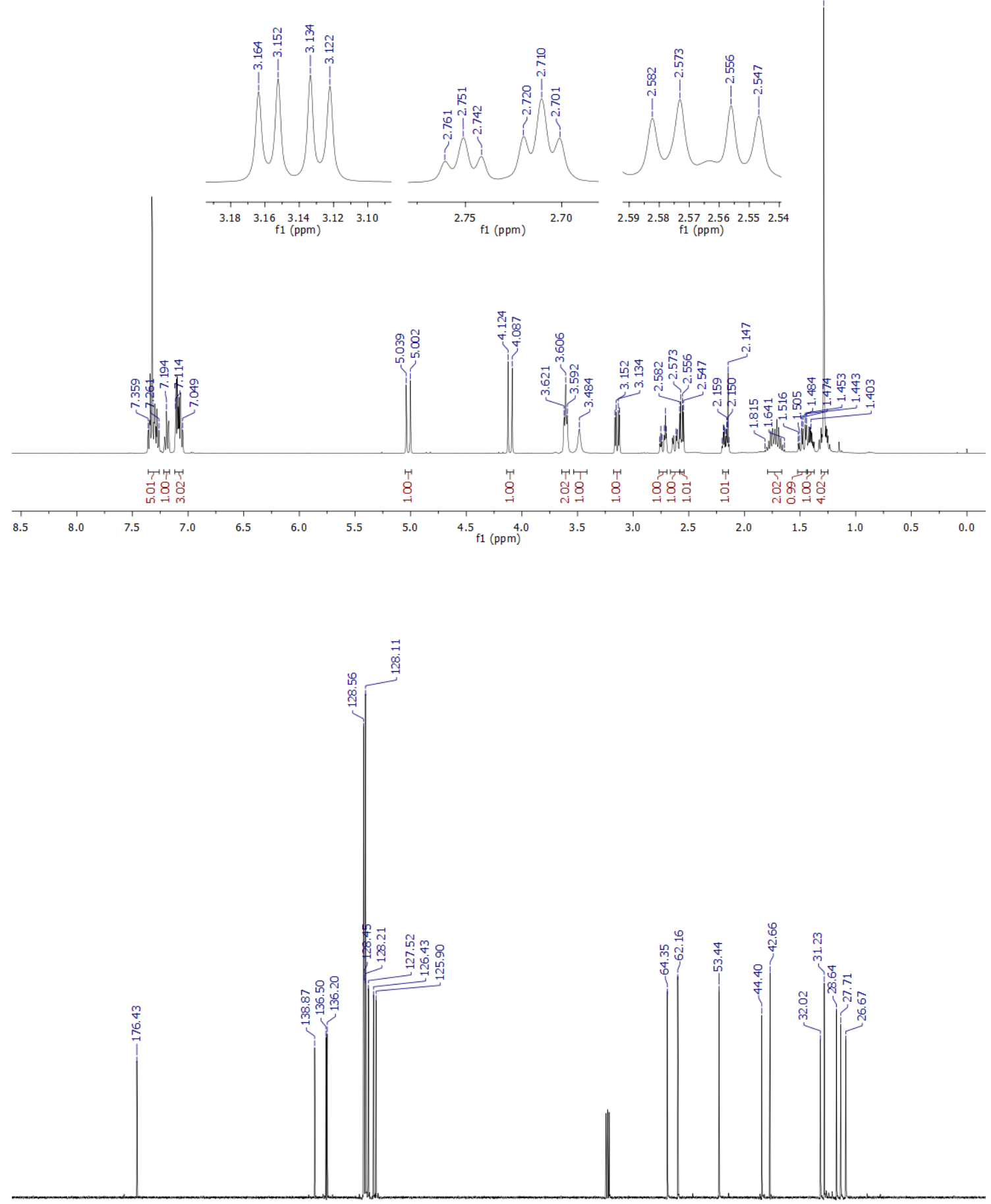

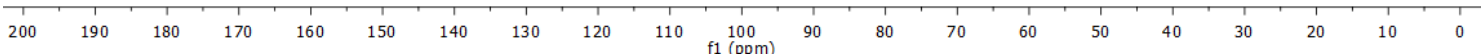


M400AFF_16112018_SJ-581-H1

M400F / Num.Inv. 1009191
cdcl3 / Temp: 25 C / N.Reg: $100000000 \times x$

Usuari: san / Mostra: SJ-581 Pf 78
Nom: SERGI JANANA GARCIA
Data: 16/11/18 / Ope.: S.JANSANA

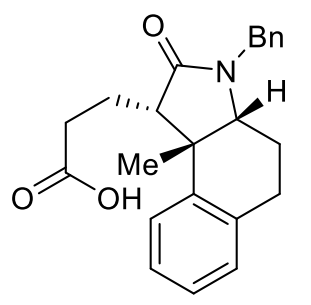

7
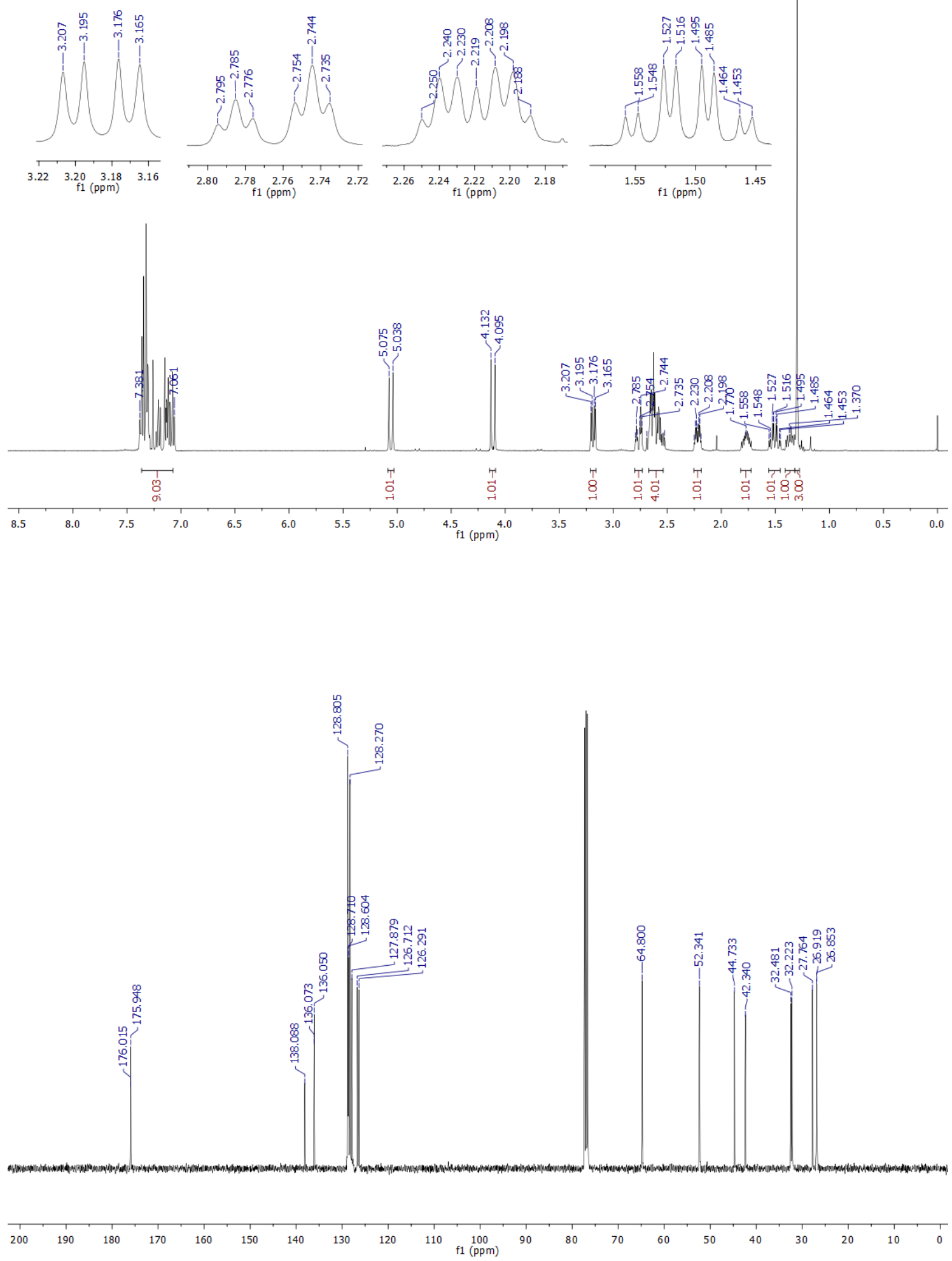

S28 
VNMRS400A_20112018_SJ-586_Pf_28-H1 VNMRS400F / Num.Inv. 205984 cdc13 / Temp: 25C/N.Reg: $X X X X X X X X X$

Usuari: san / Mostra: SJ-586_Pf_28

Data: 20/11/18 / Ope.: S.JANSANA<smiles>C[C@]12CC[C@H]3C(=O)N(Cc4ccccc4)[C@@H]3CCC(=O)c3cccc1c32</smiles>

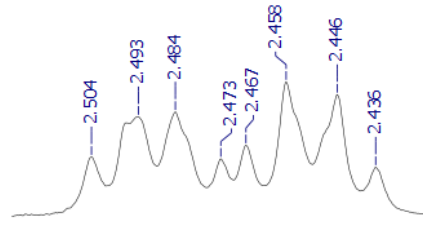

$2.522 .512 .502 .492 .48 \quad 2.472 .462 .45 \quad 2.442 .43$

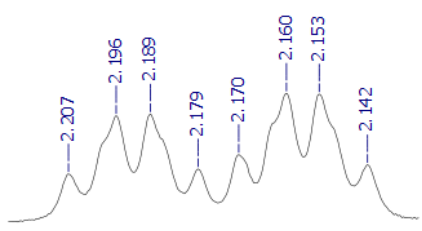

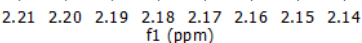
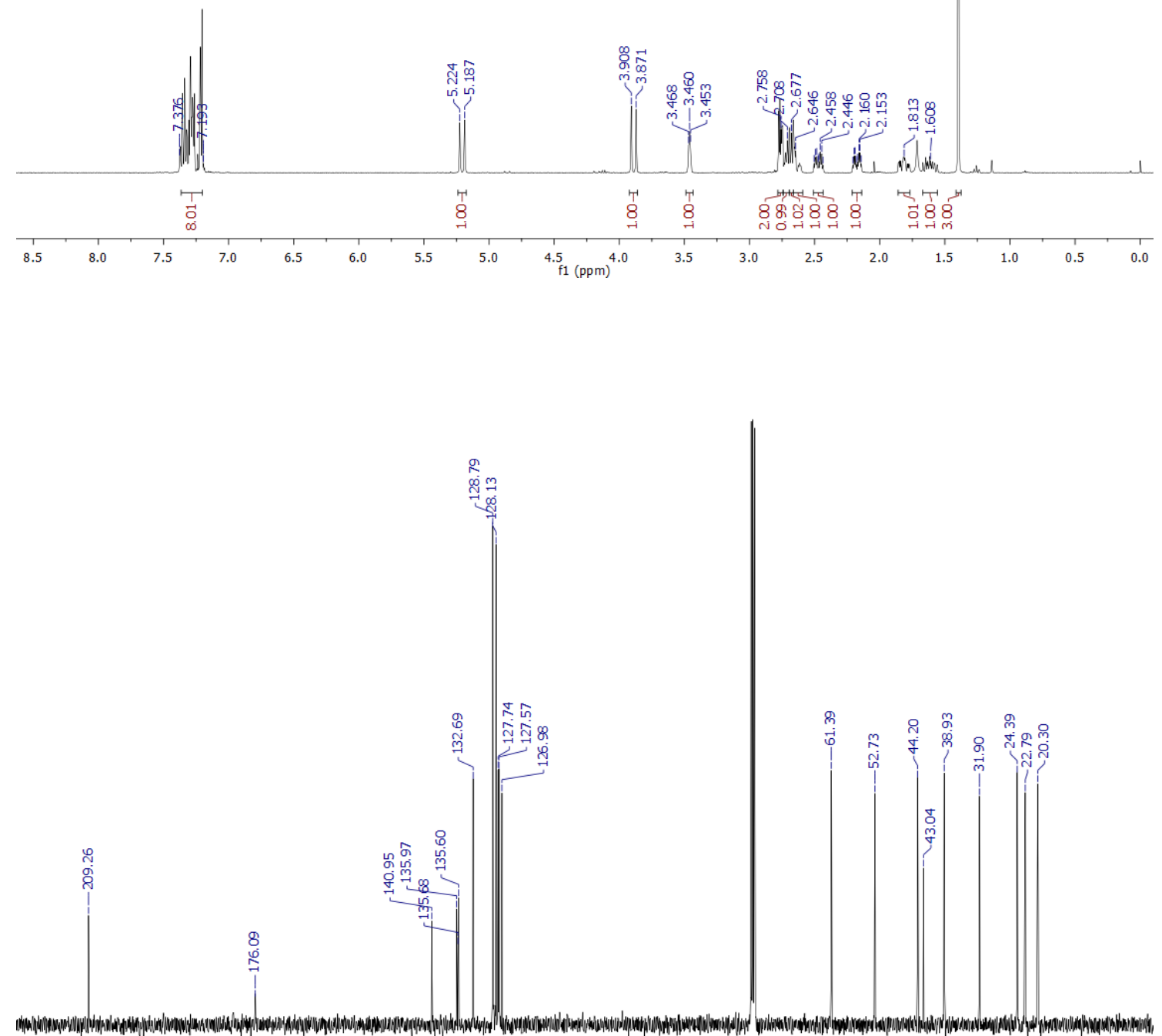

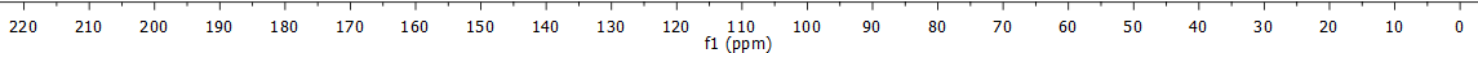


M400AFF_27042017_SJ-293-H1_rep_19_34_30 M400F/ Num.Inv. 1009191

dd13 / Temp: 25C/N.Reg: $X x x x x x x x$

Usuari: san / Mostra: SJ-293 Pf

Data: 27/04/17 / Ope.: S.JANSANA

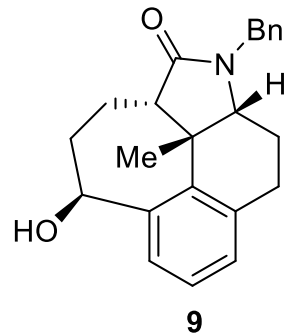
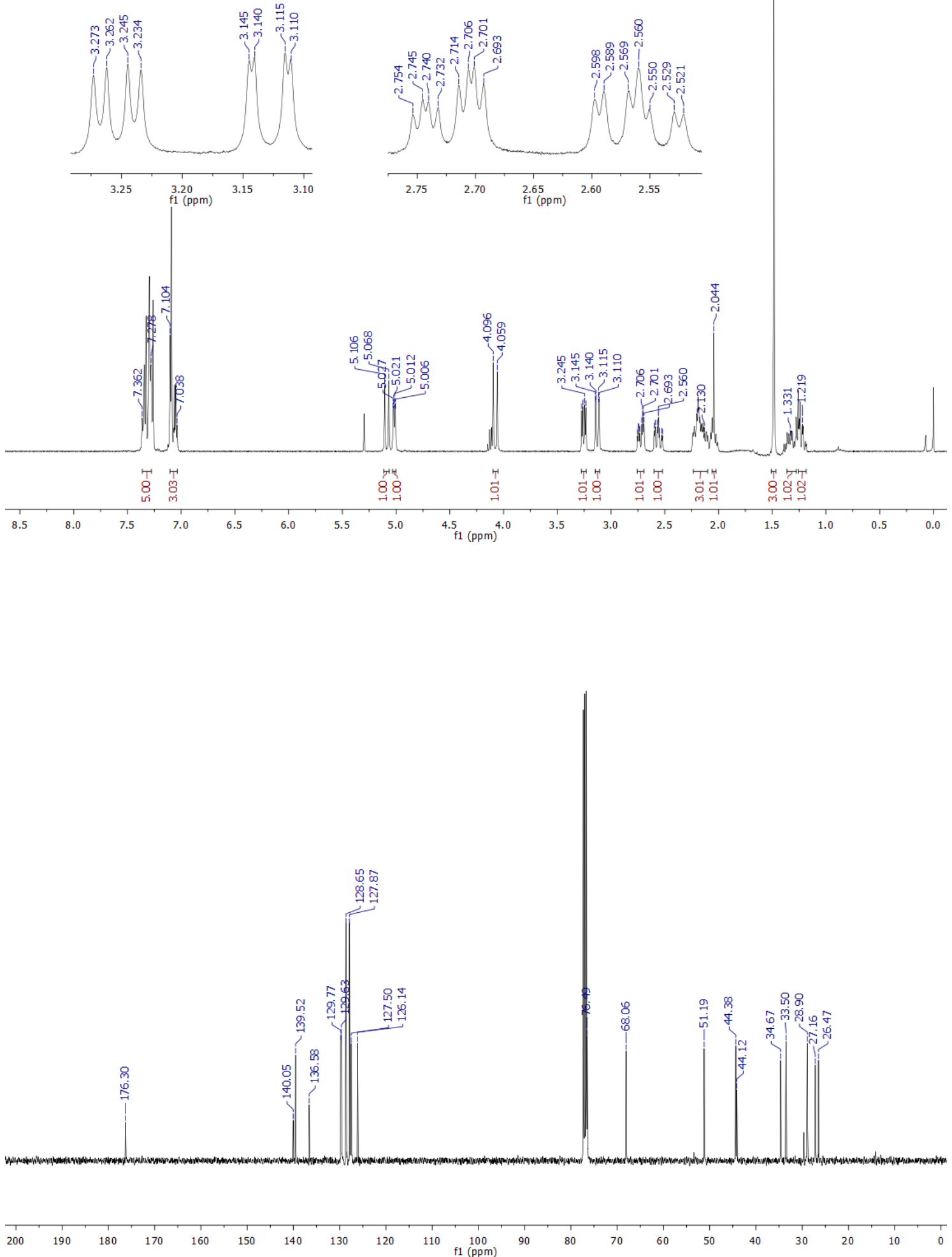
VNMRS400A_02102017_SJ-359_Pf_A-H1 VNMRS400F / Num.Inv. 205984

cdc13/Temp: 25C/N.Reg: $\times D X X X X X X X$

Usuari: san /Mostra: SJ-359_Pf_A

Data: 02/10/17 / Ope.: S.JANSANA<smiles>CSC(=S)O[C@H]1CC[C@H]2C(=O)N(Cc3ccccc3)[C@@H]3CCc4cccc1c4[C@@]23C</smiles>
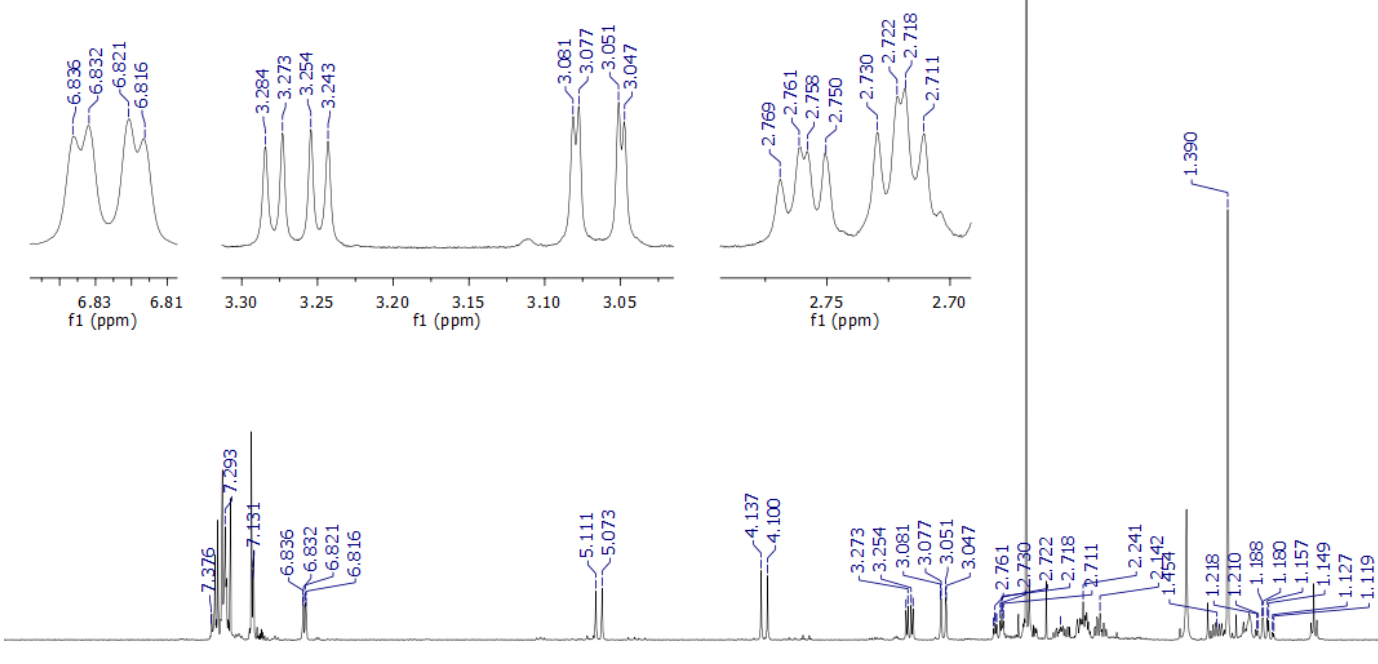

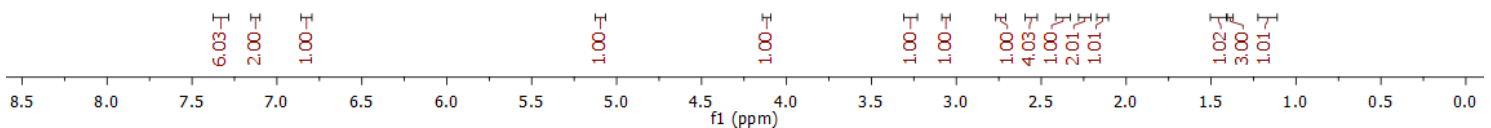

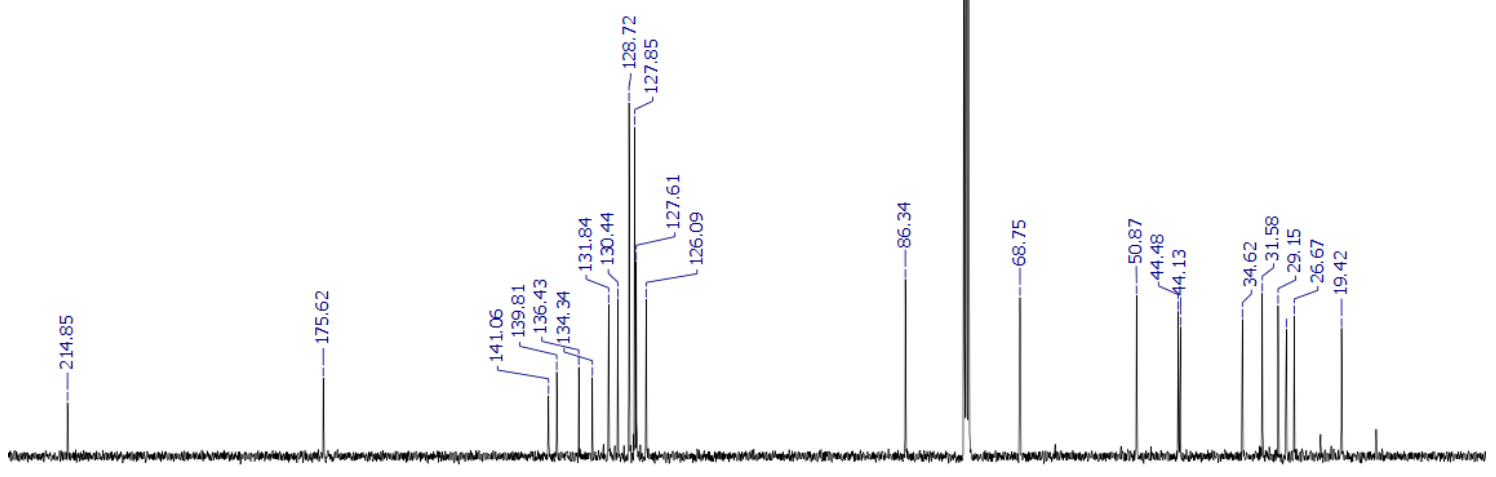

220

200

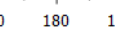

160

140

$120 \underset{\mathrm{f} 1(\mathrm{ppm})}{110} 1$ 
VNMRS400A_06072017_SJ-229_Pf_B-H1 NNMRS400F / Num.Inv. 205984

Usuari: san / Mostra: SJ-229 Pf B

Nom: SERGI JANSANA GARCIA

Data: 06/07/17 / Ope.: S.JANSANA

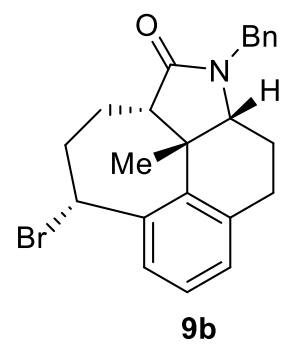
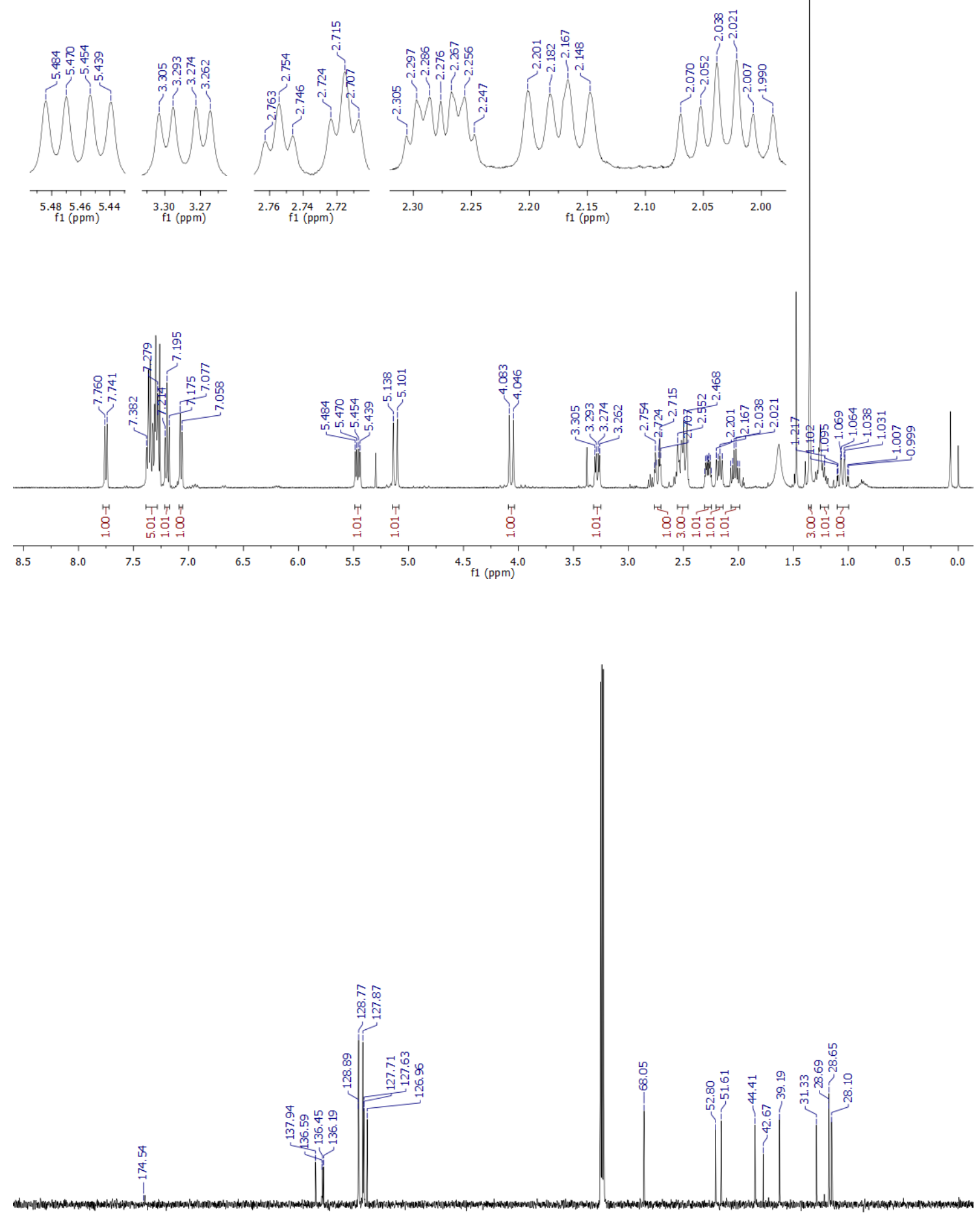

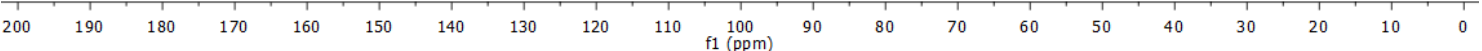


VNMRS400A_07072017_SJ-329_Pf_AA-H VNMRS400F / Num.Inv. 205984

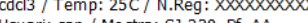

NOm: SERGI JANSANA GARCIA-

Data: 07/07/17 / Ope.: S.JANSANA

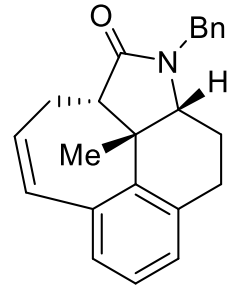

10
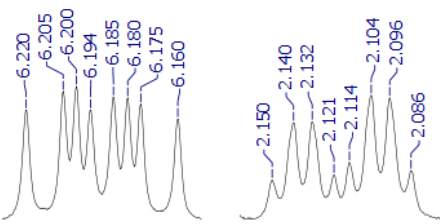

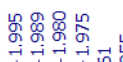

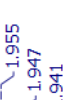
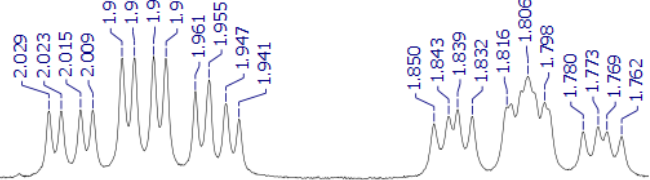
$6.22 \underset{\substack{6 \\ \mathrm{f} 1(\mathrm{ppm})}}{6.20} 6.18$

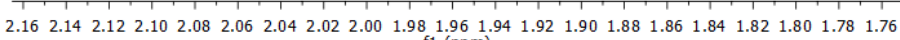
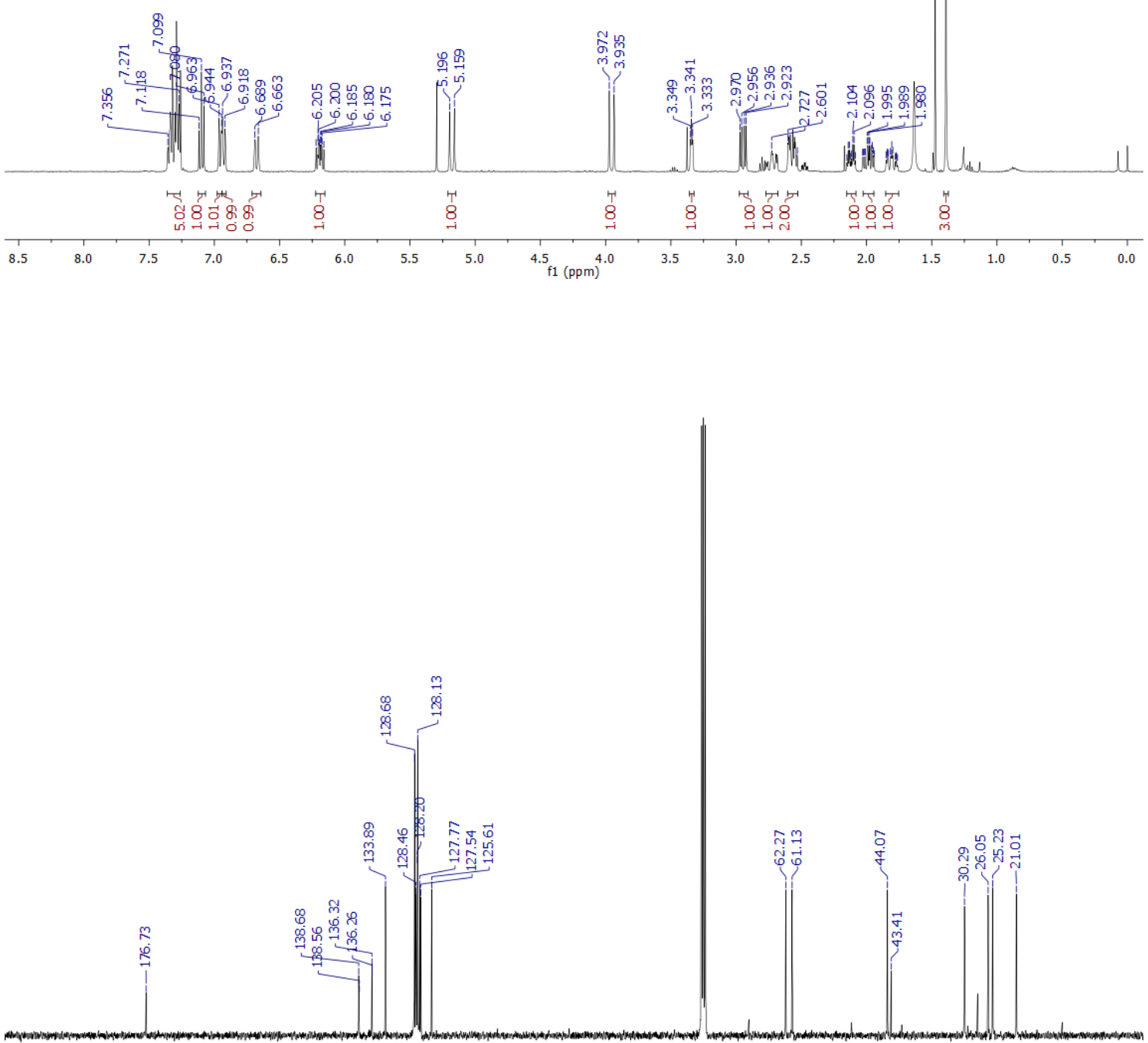
M400AFF_05122018_SJ-590-H1 M400F/ Num.Inv. 1009191

cdc13/ Temp: 25C/ N.Reg: $\times 0000000 X$

Nom: SERGI JANSANA GARCIA

Data: 05/12/18/Ope.: S.JANSANA

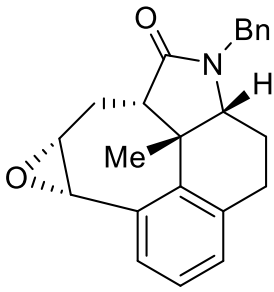

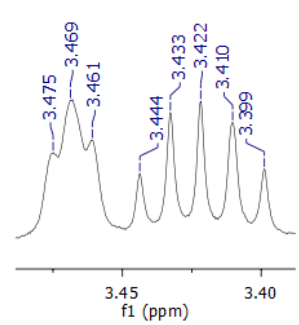

$10 \mathrm{a}$
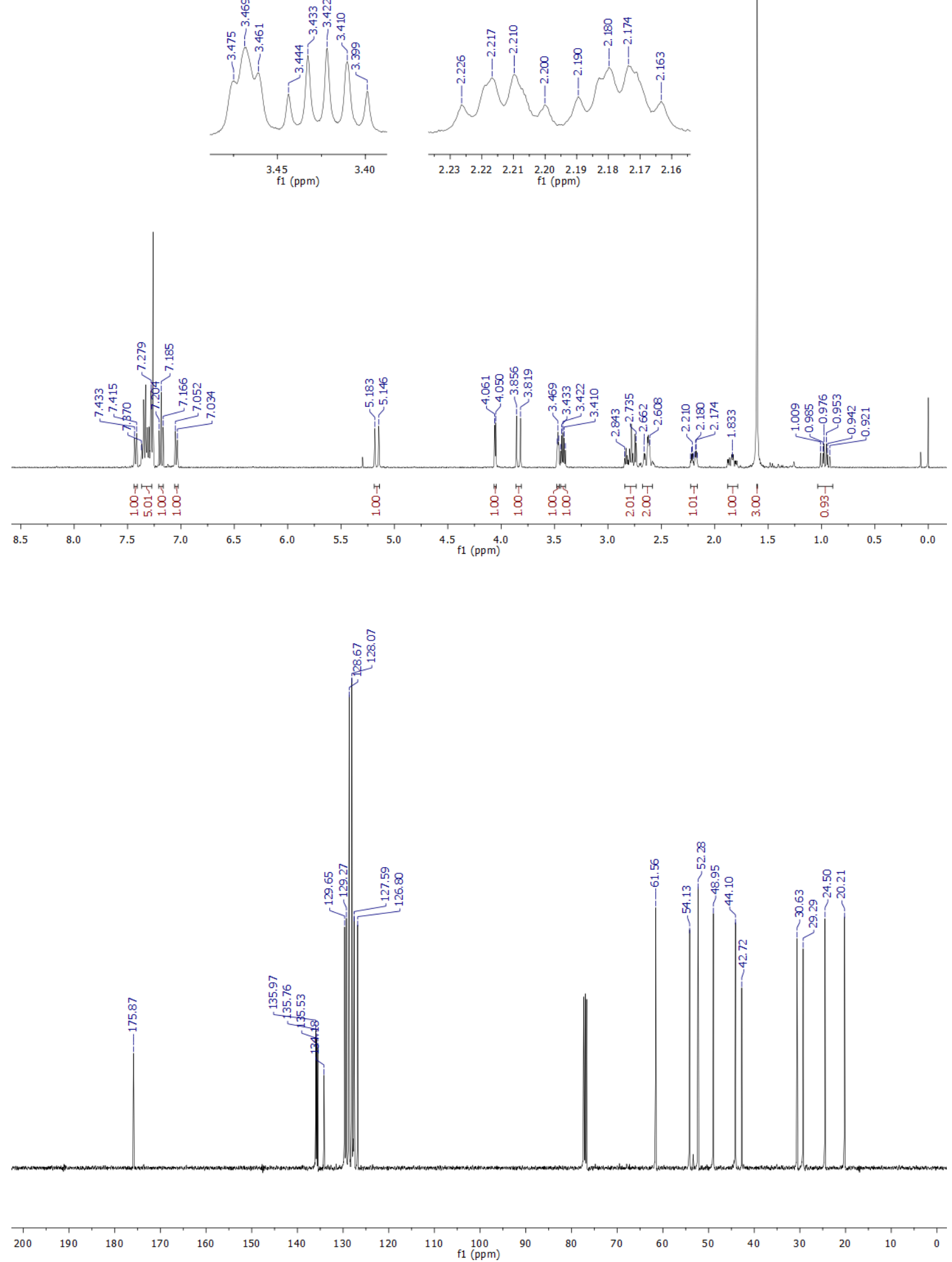
VNMRS400A_14032019_SJ-395_Cru_2-H1 NNMRS400F / Num.Inv. 205984

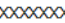

5_Cru_

D.m: SERGI JANSANA GARCIA<smiles>C=CC(O)CC[C@H]1C(=O)N(Cc2ccccc2)[C@@]2(C)c3ccccc3CC[C@]12C</smiles>

11
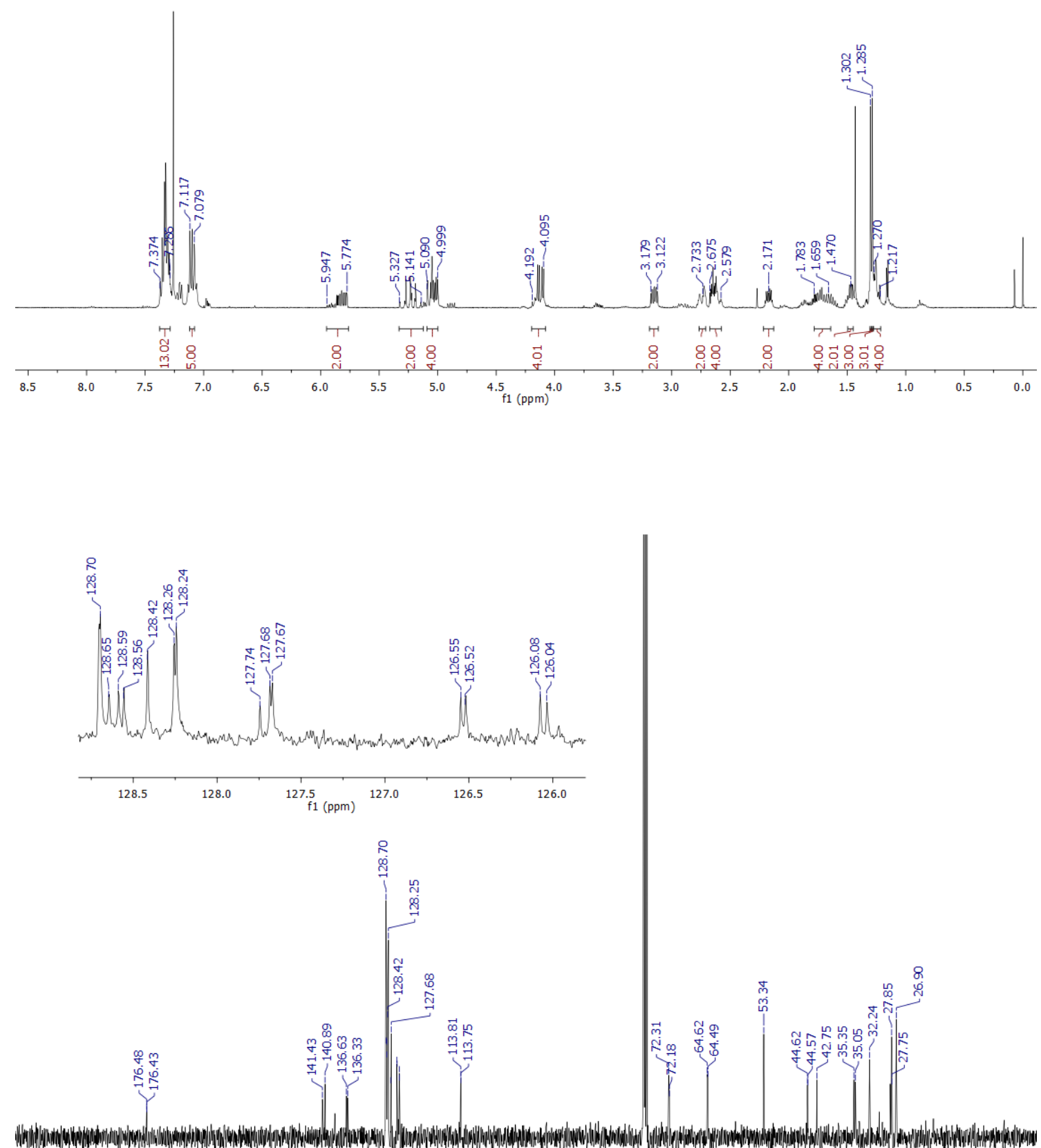

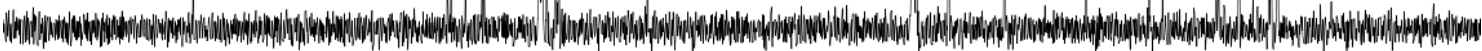

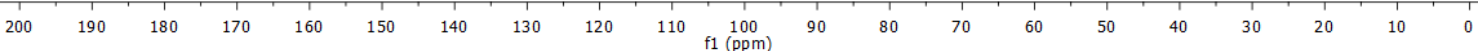


VNMRS400A_01122017_SJ-398_402_Cru-H1 VNMRS400F / Num.Inv. 205984

Temp: 25C/ N.Reg: $X_{0} 000000 x$

San

Data: 01/12/17 / Ope.: S.JANSAIA

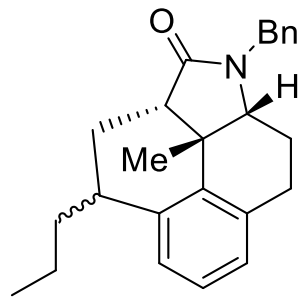

$12 b$

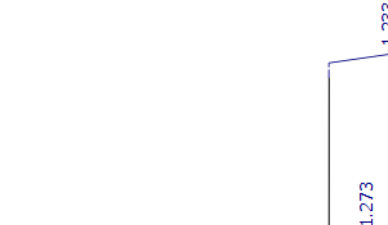

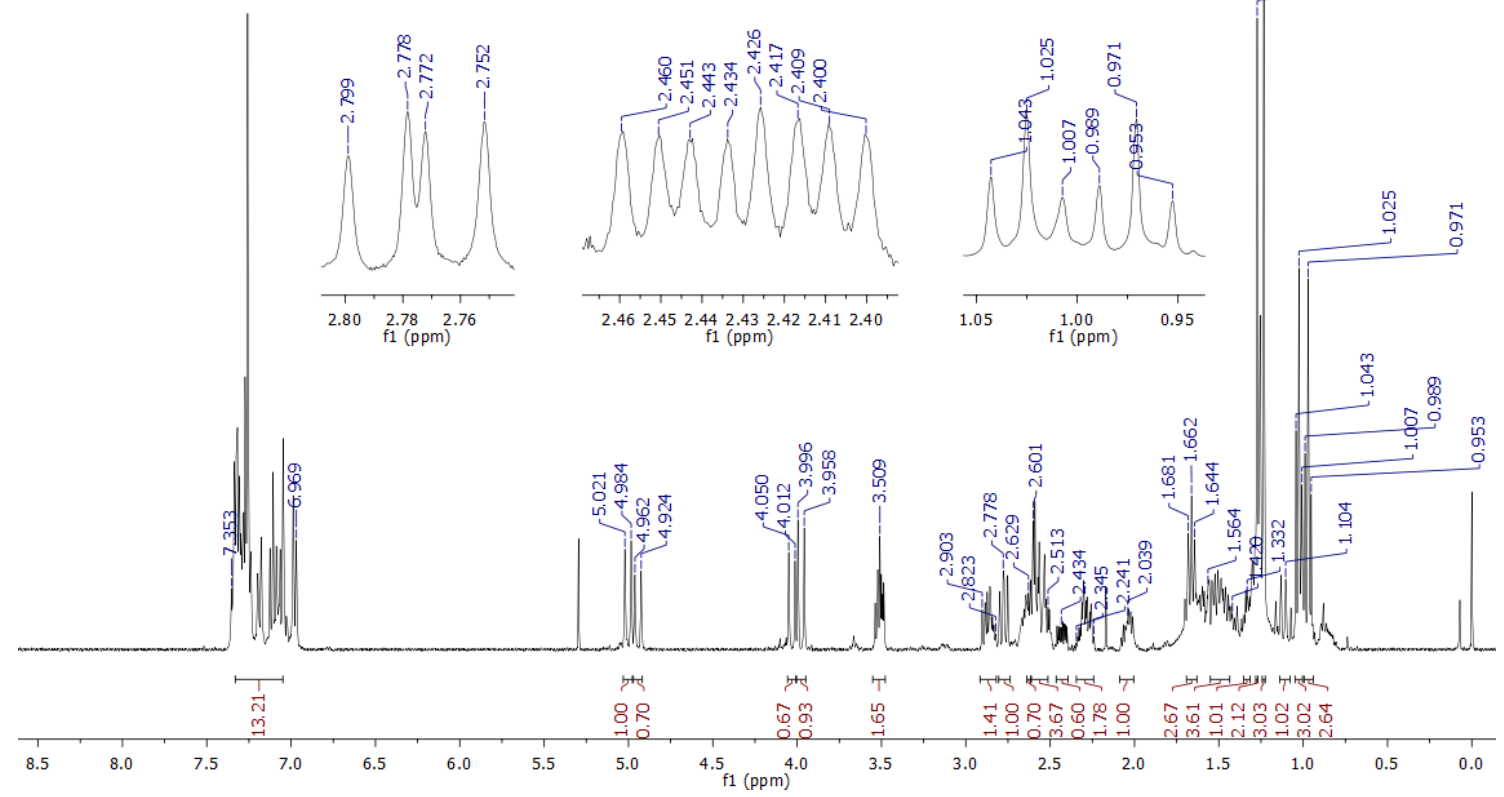

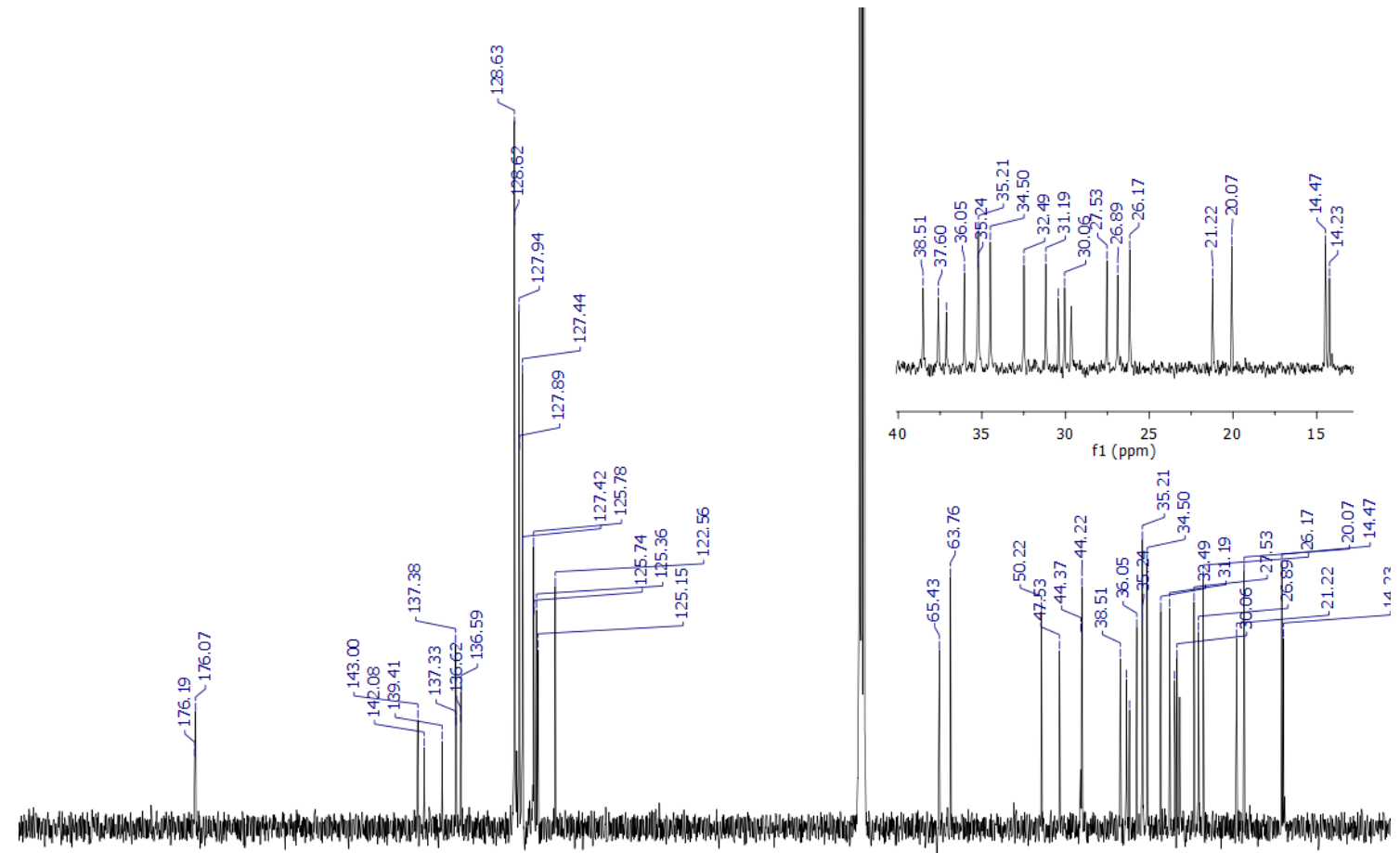

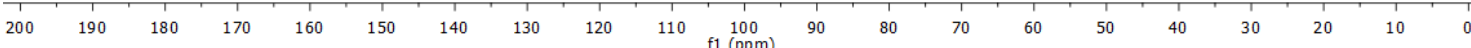


VNMRS400A_13062018_SJ-516-19-20_Pf_82-H1 VNMRS400F/ Num.Inv. 205984

dd3/Temp: $25 \mathrm{C} /$ N.Reg: $X x x \times x x x x x$

Usuarn: san / Mostra: SJ-516-19-20_Pf_82

Data $13 / 06 / 10 /$ Op S SJAIS<smiles>[M][C@]12CCC(=O)Cc3cccc(c31)CC[C@H]2NCc1ccccc1</smiles>

13
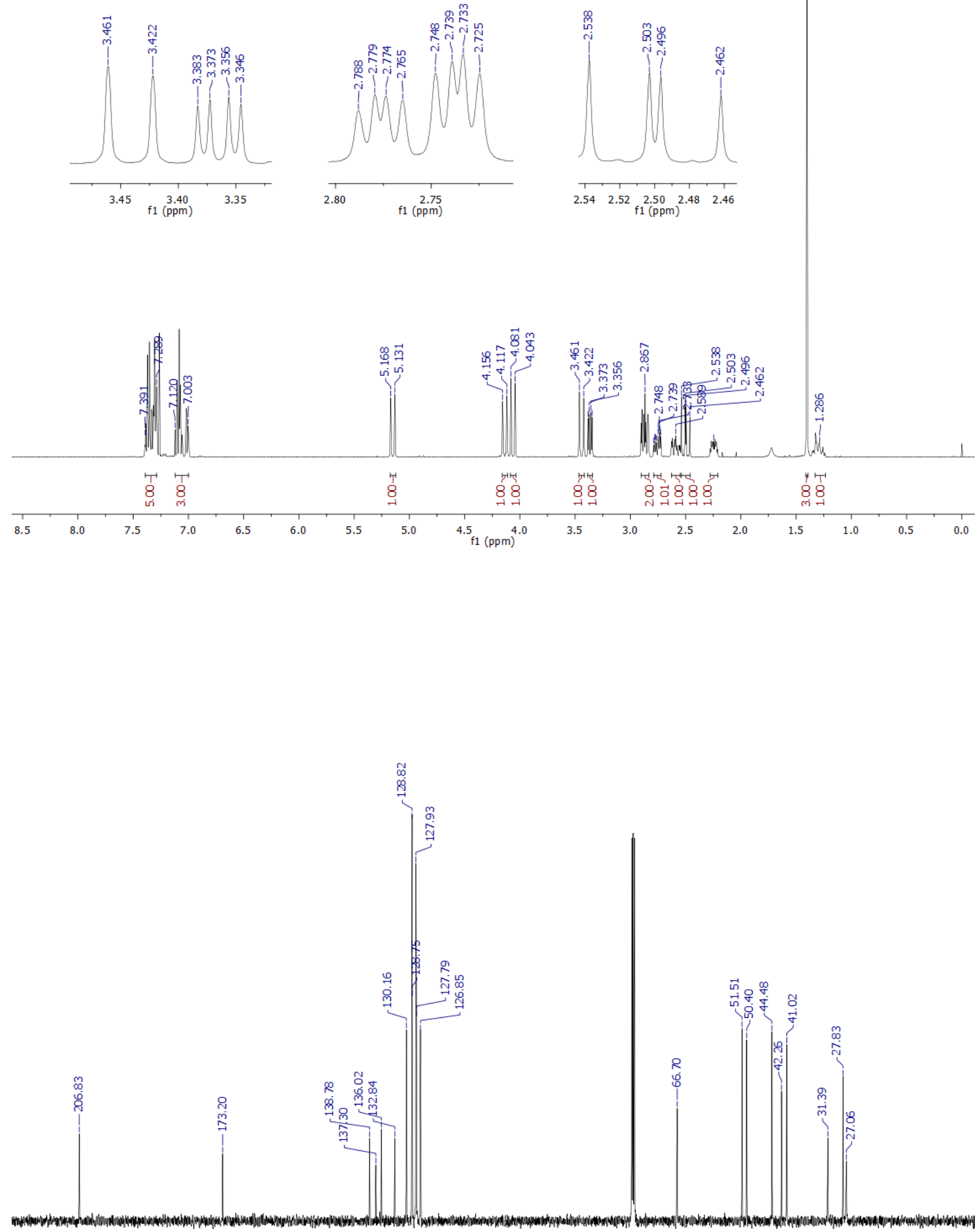

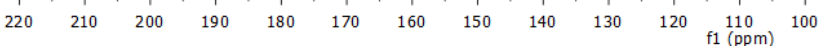


M400AFF_10122018_SJ-593-94-H1 M400F/Num.Inv. 1009191

CDCl3/Temp: 25C/N.Reg: $\times 0 X 000 X 0 X$

Usuari: san / Mostra: SJ-593-94 Pf B1

Nom: SERGI JANSANA GARCIA<smiles>CCCCOC(=O)C[C@H]1C(=O)C[C@H]2C(=O)N(Cc3ccccc3)[C@H]3CCc4cccc1c4[C@]32C</smiles>

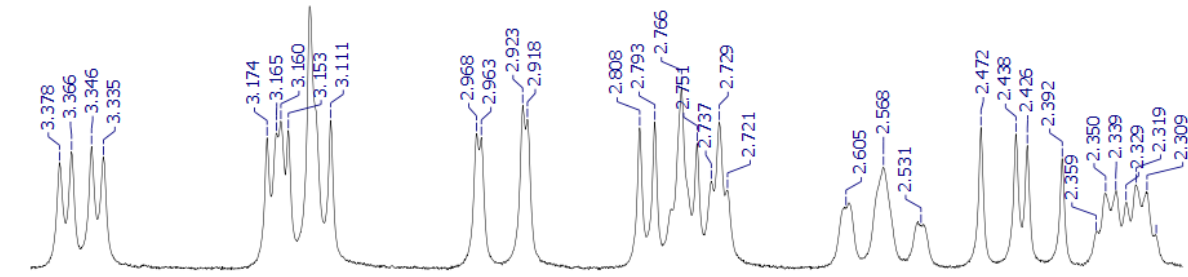

$\begin{array}{llllllllllllllllllllllll}3.40 & 3.35 & 3.30 & 3.25 & 3.20 & 3.15 & 3.10 & 3.05 & 3.00 & 2.95 & 2.90 & 2.85 & 2.80 & 2.75 & 2.70 & 2.65 & 2.60 & 2.55 & 2.50 & 2.45 & 2.40 & 2.35 & 2.30\end{array}$

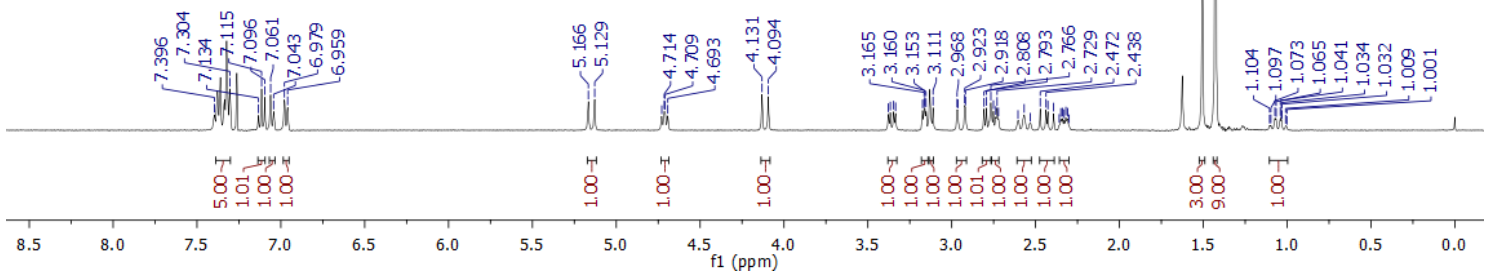

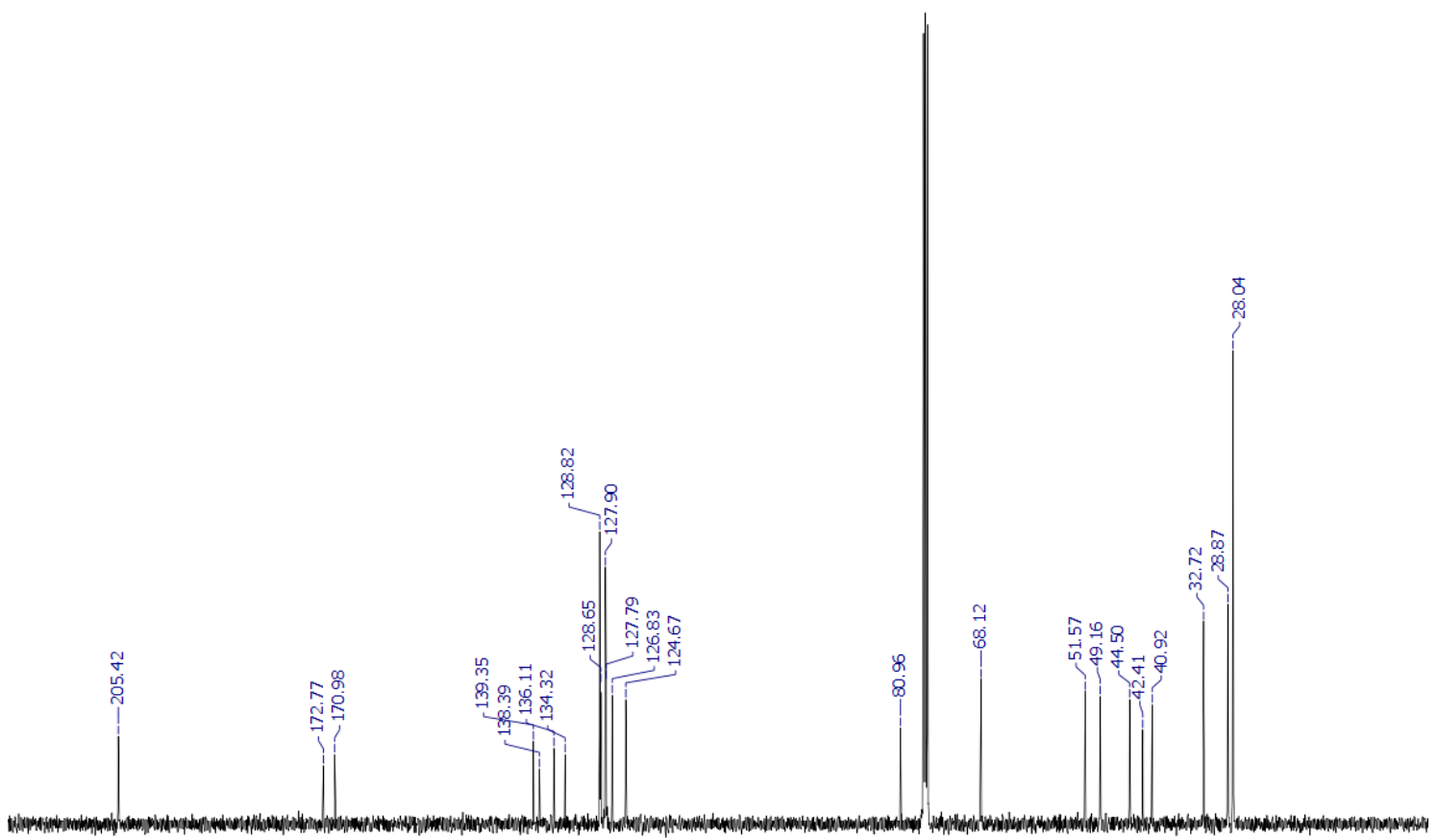

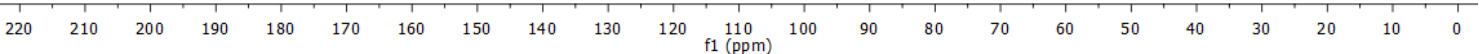


VNMRS400A_12122018_SJ-595-96_Pf_C-H1 VNMRS400F / Num.Inv. 205984

Usuari: san / Mostra: SJ-595-96_Pf C

Nom: SERGI JANSANA GARCIA

Data: 12/12/18 / Ope.: S.JANSANA
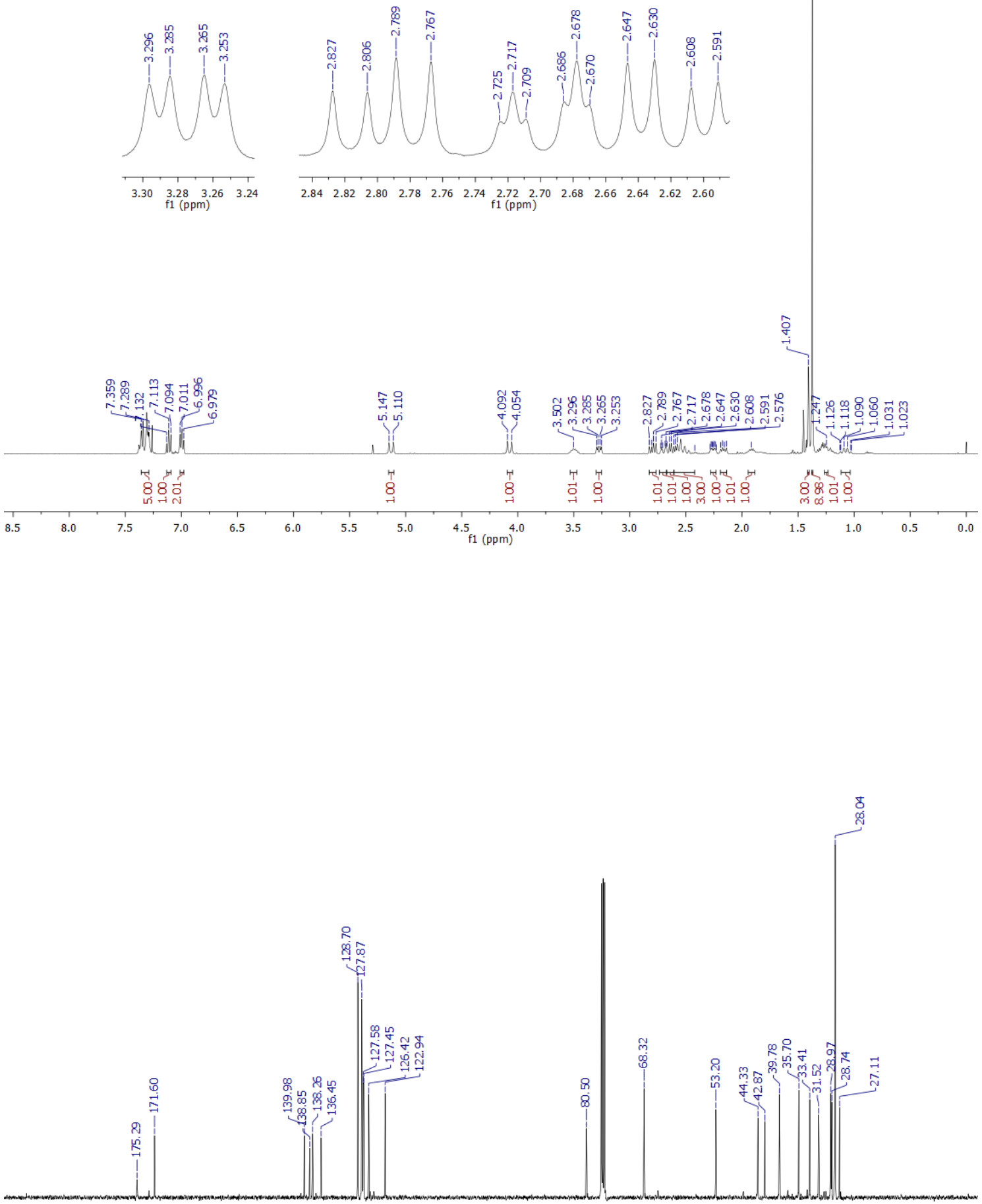

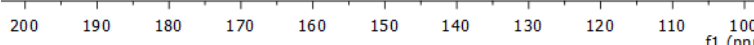


4400AFF_14012019_SJ-601_Pf_B-H1

M400F / Num.Inv. 1009191

Usuari: san / Mostra: SJ-601_Pf_B
Nom: SERGI JANSANA GARCIA
Data: 14/01/19 / Ope.: S.JANSANA
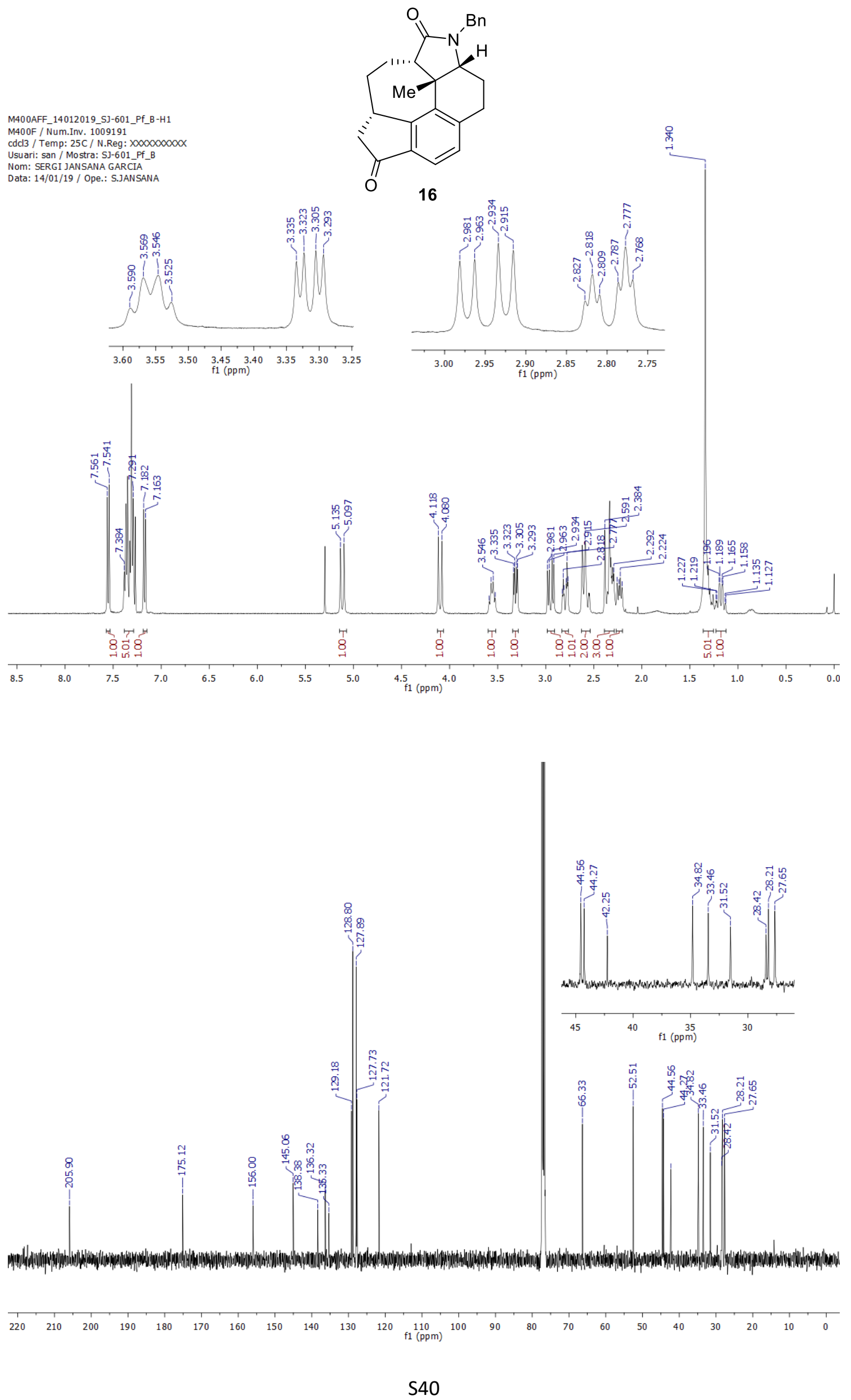
VNMRS400A_14012019_SJ-601_Pf_A-H1 VNMRS400F / Num.Inv. 205984

Jal

NOM: SERGIJANSANA GARCIA-

Data: 14/01/19/Ope. S.JANSANA

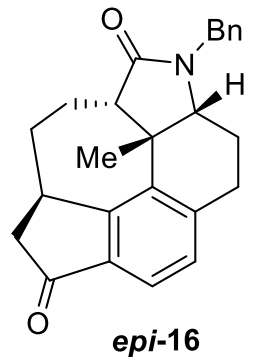
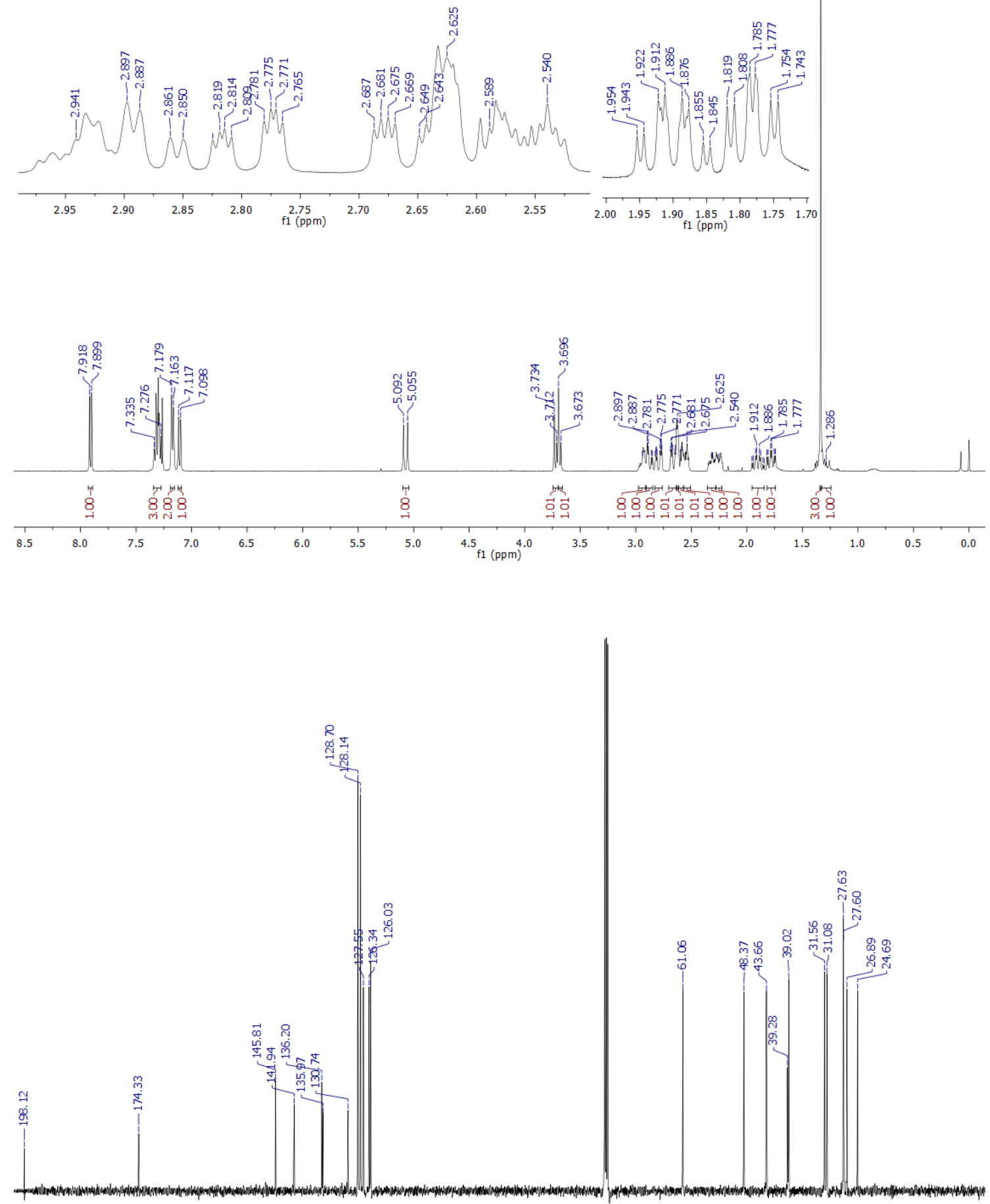

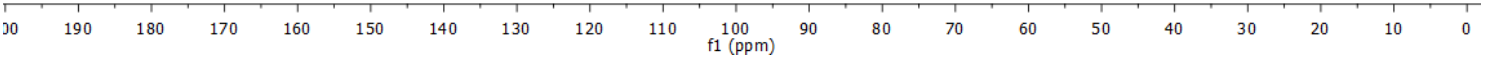


M400AFF_09042019_SJ-629-H1 M400F / Num.Inv. 1009191

CDCl3/Temp. 25C/N.Reg: $\times \infty \times \infty \times \infty \times \infty$

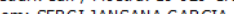

Data: 09/04/19/Ope.: SJANSANA

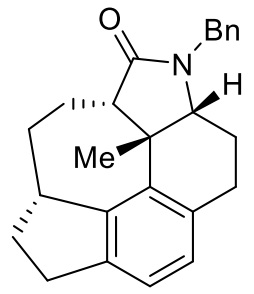

17
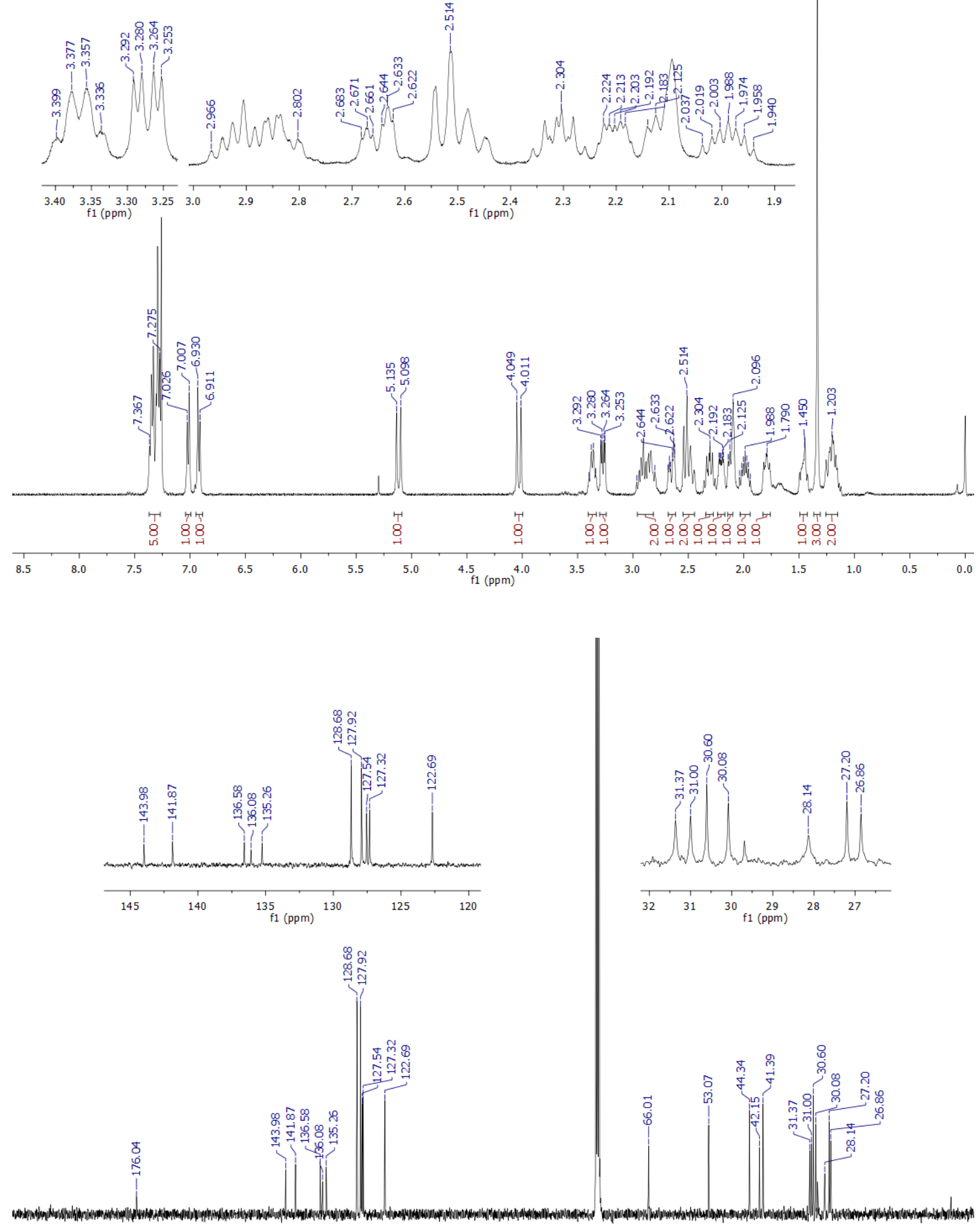

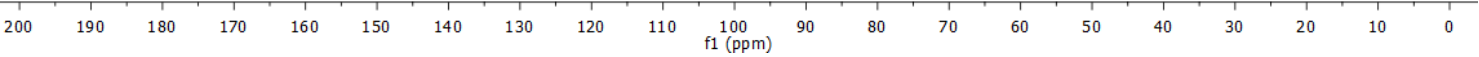


M400AFF_10042019_SJ-630-H1 M400F / Num.Inv. 1009191 Usuari: san / Mostra: SJ-630 Cru

Nom: SERGI JANSANA GARCIA

Data: $10 / 04 / 19 /$ Ope.: S.JANSANA

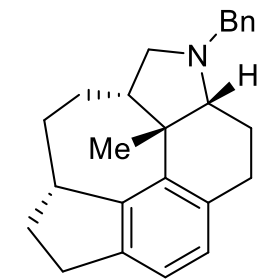

18
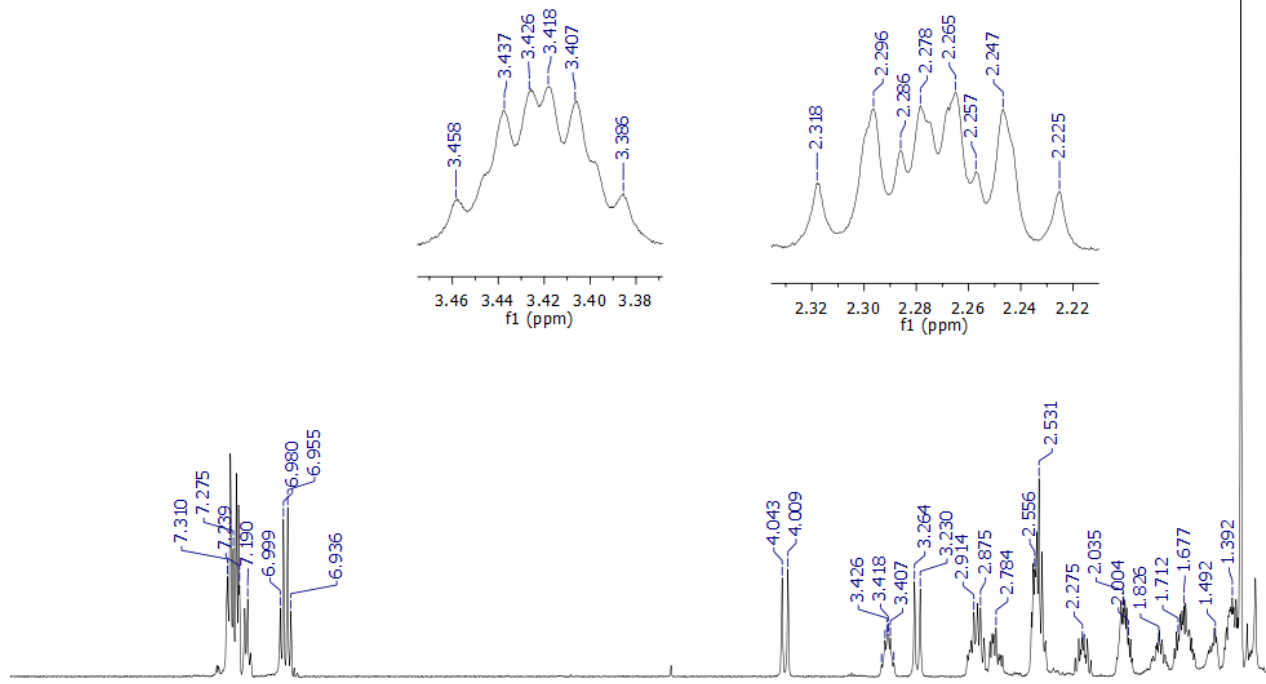

8888 8 का 88888888888888

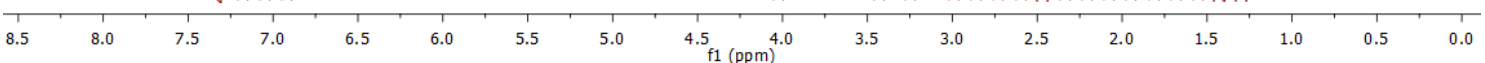

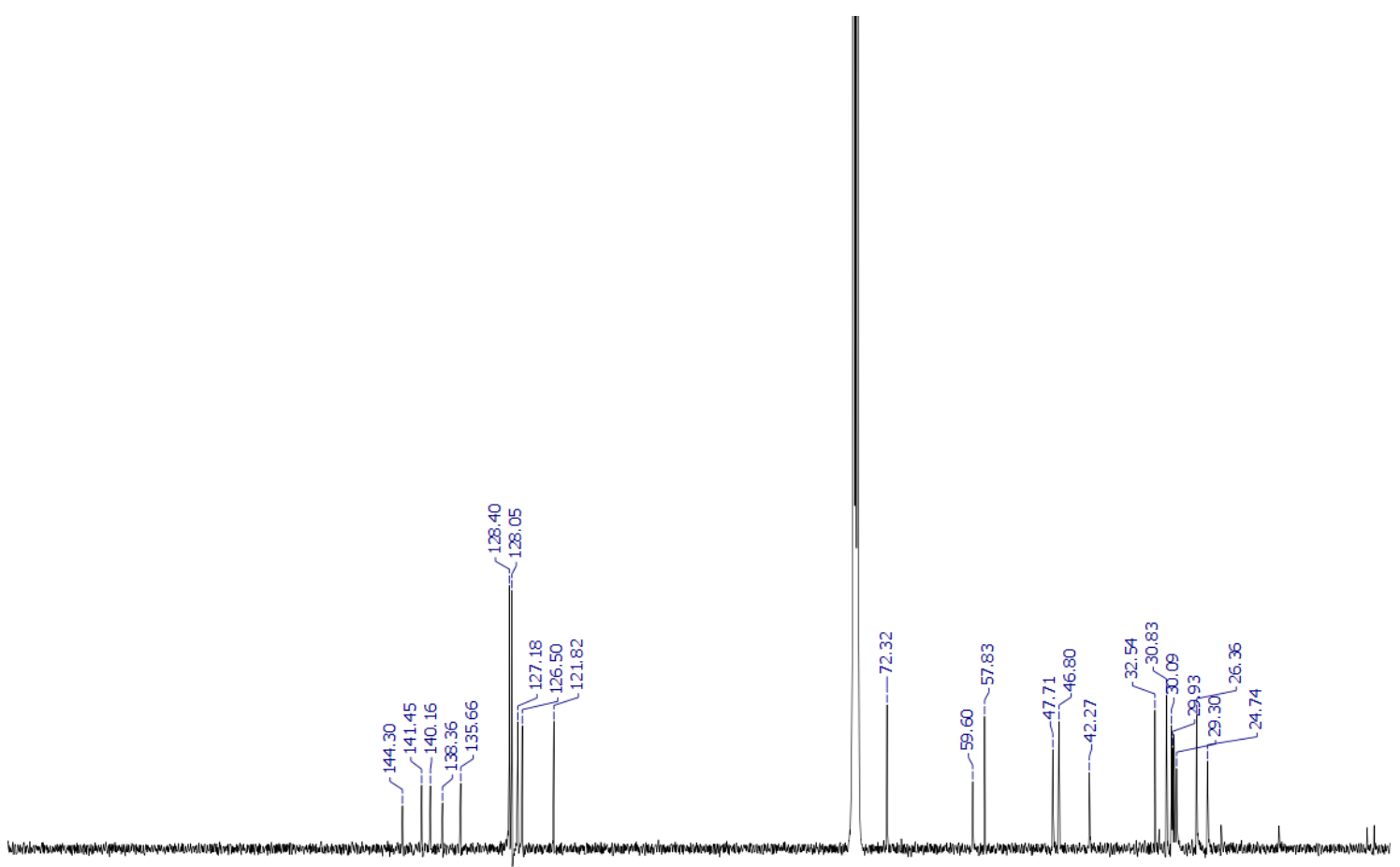

